\title{
Foundations of Spline Theory: B-Splines, Spline Approximation, and Hierarchical Refinement
}

\author{
Tom Lyche, Carla Manni, and Hendrik Speleers
}

\begin{abstract}
This chapter presents an overview of polynomial spline theory, with special emphasis on the B-spline representation, spline approximation properties, and hierarchical spline refinement. We start with the definition of B-splines by means of a recurrence relation, and derive several of their most important properties. In particular, we analyze the piecewise polynomial space they span. Then, we present the construction of a suitable spline quasi-interpolant based on local integrals, in order to show how well any function and its derivatives can be approximated in a given spline space. Finally, we provide a unified treatment of recent results on hierarchical splines. We especially focus on the so-called truncated hierarchical B-splines and their main properties. Our presentation is mainly confined to the univariate spline setting, but we also briefly address the multivariate setting via the tensor-product construction and the multivariate extension of the hierarchical approach.
\end{abstract}

\section{Introduction}

Splines, in the broad sense of the term, are functions consisting of pieces of smooth functions glued together in a certain smooth way. Besides their theoretical interest, they have application in several branches of the sciences including geometric modeling, signal processing, data analysis, visualization, numerical simulation, and probability, just to mention a few. There is a large variety of spline species, often referred to as the zoo of splines. The most popular species is the one where the pieces are algebraic polynomials and inter-smoothness is imposed by means of equality of

Tom Lyche

Department of Mathematics, University of Oslo, Norway

e-mail: tom@math.uio.no

Carla Manni, Hendrik Speleers

Department of Mathematics, University of Rome "Tor Vergata", Italy

e-mail: manni@mat.uniroma2.it, speleers@mat.uniroma2.it 
derivatives up to a given order. This species will be the topic of the chapter. Several other species can be found in $[35,45]$ and references therein.

To efficiently deal with splines, one needs a suitable basis for their representation. B-splines turn out to be the most useful spline basis functions because they possess several properties that are important from both theoretical and computational point of view. The construction of B-splines is not confined to the algebraic polynomial case but can be done for many species in the zoo of splines. As it is often the case for important tools or concepts, B-splines have a long history in the sciences. They were already used by Laplace in the early nineteenth century [33], and many of their relevant properties were derived by Chakalov and Popoviciu in the 1930's; see [10] and [37]. However, the modern B-spline theory roots in the seminal works by Schoenberg; see [41, 42] and $[15,16]$. There are several ways to define B-splines, based on recurrence, differentiation, divided differences, etc. Each of those definitions has certain advantages according to the problem one has to face. It is impossible to trace all modern works on B-splines, but we refer the reader to [45] for an extended bibliography on the topic also beyond the polynomial setting.

This chapter provides an introduction to (polynomial) B-splines, starting from their definition via a recurrence relation. Furthermore, we establish some spline results of interest within the isogeometric analysis (IgA) paradigm. More precisely, the chapter contains

- a comprehensive and self-contained overview of splines and B-splines;

- a constructive exploration of approximation properties of spline spaces;

- a discussion on adaptive spline representations based on hierarchical refinement.

There exists a huge amount of literature about the first two items including some well-established books; see, e.g., $[6,26,45]$ and references therein. The hierarchical spline setting received only recently a lot of attention; see, e.g., [22, 51, 53]. The novelties of the chapter can be essentially summarized as follows.

- Our introduction to B-splines differs somewhat from the standard presentations of the topic. It is mainly based on properties of the dual polynomial functions in the local Marsden identity.

- Our proof of the approximation properties of a given spline space relies on the explicit construction of a spline quasi-interpolant based on local integrals. For this quasi-interpolant we show error estimates of optimal order to any smooth function and its derivatives.

- Our presentation of the hierarchical spline setting provides a rather complete and unified treatment of the main properties of both the hierarchical and the truncated hierarchical B-spline basis.

The chapter does not address the geometric modeling aspects of B-splines, explaining why they form the mathematical core of current computer aided design (CAD) systems. For this we refer the reader to the books [13, 27, 38].

Our presentation is mainly confined to the univariate spline setting. Nevertheless, this is the building block of the multivariate setting via the tensor-product construction. Tensor-product B-splines are currently the most common tool in CAD systems 
and $\operatorname{IgA}$. It is worth mentioning that there are also many other important extensions of the univariate B-spline concepts to the multivariate setting, not restricted to a tensor-product grid; see, for example, [31,35] and references therein.

The remaining part of the chapter is divided into six sections. The next section is devoted to the definition of B-splines and their main properties, including differentiation and integration formulas, local representation of polynomials, and local linear independence. In Section 3 we analyze the space spanned by a set of B-splines also discussing the representation of its elements, knot insertion, and the stability of the B-spline basis. Cardinal B-splines, i.e., B-splines with uniform knots, are of prominent interest in practical applications. They are addressed in Section 4 where, in particular, the evaluation of their inner products and uniform knot insertion are discussed. In Section 5, after a general discussion about quasi-interpolants, we present the construction of a new spline quasi-interpolant based on local integrals and we use it to show the approximation properties of the considered spline space. The hierarchical spline approach is the topic of Section 6, which is mainly devoted to the construction of the truncated hierarchical B-spline basis and the derivation of its main properties, including the so-called preservation of coefficients and the construction of hierarchical quasi-interpolants. Finally, tensor-product B-splines and their hierarchical extension are briefly discussed in Section 7 .

\section{B-Splines}

In this section we introduce one of the most powerful tools in computer-aided geometric design and approximation theory: B-spline functions (in short, B-splines) ${ }^{1}$. They are piecewise polynomials with a certain global smoothness. The positions where the pieces meet are known as knots.

\subsection{Definition and Basic Properties}

In order to define B-splines we need the concept of knot sequences.

Definition 1. A knot sequence $\xi$ is a nondecreasing sequence of real numbers,

$$
\boldsymbol{\xi}:=\left\{\xi_{i}\right\}_{i=1}^{m}=\left\{\xi_{1} \leq \xi_{2} \leq \cdots \leq \xi_{m}\right\}, \quad m \in \mathbb{N} .
$$

The elements $\xi_{i}$ are called knots.

Provided that $m \geq p+2$ we can define B-splines of degree $p$ over the knotsequence $\boldsymbol{\xi}$.

\footnotetext{
${ }^{1}$ The original meaning of the word "spline" is a flexible ruler used to draw curves, mainly in the aircraft and shipbuilding industries. The "B" in B-splines stands for basis or basic.
} 
Definition 2. Suppose for a nonnegative integer $p$ and some integer $j$ that $\xi_{j} \leq$ $\xi_{j+1} \leq \cdots \leq \xi_{j+p+1}$ are $p+2$ real numbers taken from a knot sequence $\boldsymbol{\xi}$. The $j$-th B-spline $B_{j, p, \xi}: \mathbb{R} \rightarrow \mathbb{R}$ of degree $p$ is identically zero if $\xi_{j+p+1}=\xi_{j}$ and otherwise defined recursively by ${ }^{2}$

$$
B_{j, p, \boldsymbol{\xi}}(x):=\frac{x-\xi_{j}}{\xi_{j+p}-\xi_{j}} B_{j, p-1, \boldsymbol{\xi}}(x)+\frac{\xi_{j+p+1}-x}{\xi_{j+p+1}-\xi_{j+1}} B_{j+1, p-1, \boldsymbol{\xi}}(x),
$$

starting with

$$
B_{i, 0, \boldsymbol{\xi}}(x):= \begin{cases}1, & \text { if } x \in\left[\xi_{i}, \xi_{i+1}\right), \\ 0, & \text { otherwise. }\end{cases}
$$

Here we used the convention that fractions with zero denominator have value zero.

We start with some preliminary remarks.

- For degree 0 the $\mathrm{B}$-spline $B_{0, p, \xi}$ is simply the characteristic function of the half open interval $\left[\xi_{j}, \xi_{j+1}\right)$. This implies that a B-spline is continuous except possibly at a $\operatorname{knot} \xi$. We have $B_{j, p, \boldsymbol{\xi}}(\xi)=B_{j, p, \boldsymbol{\xi}}\left(\xi_{+}\right)$, where

$$
x_{+}:=\lim _{\substack{t \rightarrow x \\ t>x}} t, \quad x_{-}:=\lim _{\substack{t \rightarrow x \\ t<x}} t, \quad x \in \mathbb{R} .
$$

Thus a B-spline is right continuous, i.e., the value at a point $x$ is obtained by taking limits from the right.

- We also use the notation

$$
B\left[\xi_{j}, \ldots, \xi_{j+p+1}\right]:=B_{j, p, \xi},
$$

showing explicitly on which knots the B-spline depends.

- We say that a knot has multiplicity $\mu$ if it occurs exactly $\mu$ times in the knot sequence. A knot is called simple, double, triple, ... if its multiplicity is equal to $1,2,3, \ldots$, and a multiple knot in general.

Example 3. A B-spline of degree 1 is also called a linear B-spline or a hat function. The recurrence relation (2.1) takes the form

$$
B_{j, 1, \boldsymbol{\xi}}(x)=\frac{x-\xi_{j}}{\xi_{j+1}-\xi_{j}} B_{j, 0, \boldsymbol{\xi}}(x)+\frac{\xi_{j+2}-x}{\xi_{j+2}-\xi_{j+1}} B_{j+1,0, \boldsymbol{\xi}}(x),
$$

resulting in

\footnotetext{
2 The recurrence relation is due to de Boor, Cox and Mansfield [4, 14]. However, it appears already in works by Popoviciu and Chakalov in the 1930's; see [8] for an account of the early history of splines. For the modern theory of splines we refer to the seminal papers by Schoenberg [41, 42, 43] and Curry/Schoenberg $[15,16]$. In their works, B-splines were defined by divided differences of truncated power functions.
} 


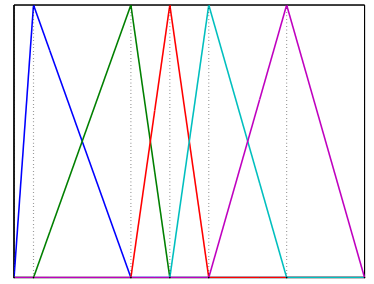

(a) $p=1$

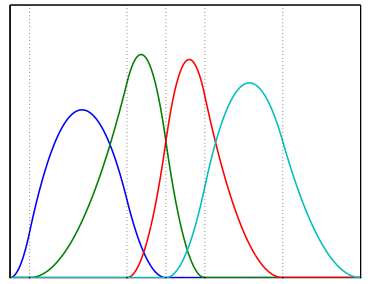

(b) $p=2$



(c) $p=3$

Fig. 1 Several sets of B-splines of degree $p=1,2,3$. The knot positions are visualized by vertical dotted lines.

$$
B_{j, 1, \boldsymbol{\xi}}(x)= \begin{cases}\frac{x-\xi_{j}}{\xi_{j+1}-\xi_{j}}, & \text { if } x \in\left[\xi_{j}, \xi_{j+1}\right), \\ \frac{\xi_{j+2}-x}{\xi_{j+2}-\xi_{j+1}}, & \text { if } x \in\left[\xi_{j+1}, \xi_{j+2}\right), \\ 0, & \text { otherwise. }\end{cases}
$$

The linear B-spline is discontinuous at a double knot and continuous at a simple knot.

Example 4. A B-spline of degree 2 is also called a quadratic B-spline. Using the recurrence relation (2.1), the three pieces of the quadratic B-spline $B_{j, 2, \xi}$ are given by

$$
B_{j, 2, \boldsymbol{\xi}}(x)= \begin{cases}\frac{\left(x-\xi_{j}\right)^{2}}{\left(\xi_{j+2}-\xi_{j}\right)\left(\xi_{j+1}-\xi_{j}\right)}, & \text { if } x \in\left[\xi_{j}, \xi_{j+1}\right), \\ \frac{\left(x-\xi_{j}\right)\left(\xi_{j+2}-x\right)}{\left(\xi_{j+2}-\xi_{j}\right)\left(\xi_{j+2}-\xi_{j+1}\right)}+\frac{\left(x-\xi_{j+1}\right)\left(\xi_{j+3}-x\right)}{\left(\xi_{j+2}-\xi_{j+1}\right)\left(\xi_{j+3}-\xi_{j+1}\right)}, & \text { if } x \in\left[\xi_{j+1}, \xi_{j+2}\right), \\ \frac{\left(\xi_{j+3}-x\right)^{2}}{\left(\xi_{j+3}-\xi_{j+1}\right)\left(\xi_{j+3}-\xi_{j+2}\right)}, & \text { if } x \in\left[\xi_{j+2}, \xi_{j+3}\right), \\ 0, & \text { otherwise. }\end{cases}
$$

Example 5. Figure 1 illustrates several sets of B-splines of degree $p=1,2,3$. The same knot sequence is chosen for the different degrees, with only simple knots.

The general explicit expression for a B-spline quickly becomes complicated. Applying the recurrence relation repeatedly we find

$$
B_{j, p, \boldsymbol{\xi}}(x)=\sum_{i=j}^{j+p} B_{j, p, \boldsymbol{\xi}}^{\{i\}}(x) B_{i, 0, \boldsymbol{\xi}}(x), \quad p \geq 0
$$

where each $B_{j, p, \boldsymbol{\xi}}^{\{i\}}$ is a polynomial of degree $p$, assumed to be zero if $\xi_{i}=\xi_{i+1}$. Note that if $\xi_{i}=\xi_{i+1}$ then $B_{i, 0, \xi}=0$ and the corresponding polynomial piece is not used. In particular, for the nontrivial cases we have 


$$
B_{j, 0, \boldsymbol{\xi}}^{\{j\}}(x)=1, \quad B_{j, 1, \boldsymbol{\xi}}^{\{j\}}(x)=\frac{x-\xi_{j}}{\xi_{j+1}-\xi_{j}}, \quad B_{j, 1, \boldsymbol{\xi}}^{\{j+1\}}(x)=\frac{\xi_{j+2}-x}{\xi_{j+2}-\xi_{j+1}} .
$$

Furthermore, for the nontrivial cases it follows from Definition 2 that the first and last polynomial pieces in (2.4) are given by

$$
\begin{aligned}
B_{j, p, \xi}^{\{j\}}(x) & =\left(x-\xi_{j}\right)^{p} / \prod_{i=1}^{p}\left(\xi_{j+i}-\xi_{j}\right), \\
B_{j, p, \boldsymbol{\xi}}^{\{j+p\}}(x) & =\left(\xi_{j+p+1}-x\right)^{p} / \prod_{i=1}^{p}\left(\xi_{j+p+1}-\xi_{j+i}\right) .
\end{aligned}
$$

Using induction on the recurrence relation (2.1), we deduce immediately the following basic properties of a B-spline.

- Local Support. A B-spline is locally supported on the interval given by the extreme knots used in its definition, i.e.,

$$
B_{j, p, \boldsymbol{\xi}}(x)=0, \quad x \notin\left[\xi_{j}, \xi_{j+p+1}\right) .
$$

- Nonnegativity. A B-spline is nonnegative everywhere, and positive inside its support, i.e.,

$$
B_{j, p, \boldsymbol{\xi}}(x) \geq 0, \quad x \in \mathbb{R}, \quad \text { and } \quad B_{j, p, \boldsymbol{\xi}}(x)>0, \quad x \in\left(\xi_{j}, \xi_{j+p+1}\right) .
$$

- Piecewise Structure. A B-spline has a piecewise polynomial structure, i.e.,

$$
B_{j, p, \xi}^{\{i\}} \in \mathbb{P}_{p}, \quad i=j, \ldots, j+p
$$

where $\mathbb{P}_{p}$ denotes the space of algebraic polynomials of degree less than or equal to $p$.

- Translation and Scaling Invariance. A B-spline is invariant under a translation and/or scaling transformation of its knot sequence, i.e.,

$$
B_{j, p, \alpha \boldsymbol{\xi}+\beta}(\alpha x+\beta)=B_{j, p, \boldsymbol{\xi}}(x), \quad \alpha, \beta \in \mathbb{R}, \quad \alpha \neq 0,
$$

where $\alpha \boldsymbol{\xi}+\beta:=\left\{\alpha \xi_{i}+\beta\right\}_{i}$.

Further properties will be considered in the next sections.

\subsection{Dual Polynomials}

To each B-spline $B_{j, p, \boldsymbol{\xi}}$ of degree $p$, there corresponds a polynomial $\psi_{j, p, \boldsymbol{\xi}}$ of degree $p$ with roots at the interior knots of the B-spline. We define $\psi_{j, 0, \xi}:=1$ and

$$
\psi_{j, p, \boldsymbol{\xi}}(y):=\left(y-\xi_{j+1}\right) \cdots\left(y-\xi_{j+p}\right), \quad y \in \mathbb{R}, \quad p \in \mathbb{N} .
$$


This polynomial is called dual polynomial. Many of the B-spline properties can be proved in an elegant way by exploiting a recurrence relation for these dual polynomials.

Theorem 6. For $p \in \mathbb{N}, x, y \in \mathbb{R}$ and $\xi_{j+p}>\xi_{j}$, we have the dual recurrence relation

$$
(y-x) \psi_{j, p-1, \boldsymbol{\xi}}(y)=\frac{x-\xi_{j}}{\xi_{j+p}-\xi_{j}} \psi_{j, p, \boldsymbol{\xi}}(y)+\frac{\xi_{j+p}-x}{\xi_{j+p}-\xi_{j}} \psi_{j-1, p, \boldsymbol{\xi}}(y),
$$

and the dual difference formula

$$
\psi_{j, p-1, \boldsymbol{\xi}}(y)=\frac{\psi_{j-1, p, \boldsymbol{\xi}}(y)}{\xi_{j+p}-\xi_{j}}-\frac{\psi_{j, p, \boldsymbol{\xi}}(y)}{\xi_{j+p}-\xi_{j}} .
$$

Proof. For fixed $y \in \mathbb{R}$ let us define the function $\ell_{y}: \mathbb{R} \rightarrow \mathbb{R}$ given by $\ell_{y}(x)=y-x$. By linear interpolation, we have

$$
\ell_{y}(x)=\frac{x-\xi_{j}}{\xi_{j+p}-\xi_{j}} \ell_{y}\left(\xi_{j+p}\right)+\frac{\xi_{j+p}-x}{\xi_{j+p}-\xi_{j}} \ell_{y}\left(\xi_{j}\right) .
$$

By multiplying both sides with $\psi_{j, p-1, \boldsymbol{\xi}}(y)$ we obtain (2.11). Moreover, (2.12) follows from (2.11) by differentiating with respect to $x$.

Proposition 7. The $r$-th derivative of the dual polynomial $\psi_{j, p, \boldsymbol{\xi}}$ for $0 \leq r \leq p$ can be bounded as follows:

$$
\left|D^{r} \psi_{j, p, \boldsymbol{\xi}}(y)\right| \leq \frac{p !}{(p-r) !}\left(\xi_{j+p+1}-\xi_{j}\right)^{p-r}, \quad \xi_{j} \leq y \leq \xi_{j+p+1} .
$$

Moreover,

$$
\left|D^{r} \psi_{j, p, \boldsymbol{\xi}}(y)\right| \leq \frac{p !}{(p-r) !}\left(\xi_{j+p}-\xi_{j+1}\right)^{p-r}, \quad \xi_{j+1} \leq y \leq \xi_{j+p} .
$$

Here we define $0^{0}:=1$ if $r=p$ and $\xi_{j+p}=\xi_{j+1}$.

Proof. Clearly (2.13) holds for all $p \in \mathbb{N}_{0}$ if $r=0$. Using induction on $r, p$ and the product rule for differentiation, we get

$$
\begin{aligned}
\left|D^{r} \psi_{j, p, \boldsymbol{\xi}}(y)\right| & =\left|D^{r}\left(\psi_{j, p-1, \boldsymbol{\xi}}(y)\left(y-\xi_{j+p}\right)\right)\right| \\
& =\left|\left(D^{r} \psi_{j, p-1, \boldsymbol{\xi}}(y)\right)\left(y-\xi_{j+p}\right)+r D^{r-1} \psi_{j, p-1, \boldsymbol{\xi}}(y)\right| \\
& \leq\left(\frac{(p-1) !}{(p-1-r) !}+r \frac{(p-1) !}{(p-r) !}\right)\left(\xi_{j+p+1}-\xi_{j}\right)^{p-r}
\end{aligned}
$$

and (2.13) follows. The proof of (2.14) is similar. 


\subsection{Local Marsden Identity and Linear Independence}

In this and the following sections (unless specified otherwise) we will extend the knots $\xi_{j} \leq \cdots \leq \xi_{j+p+1}$ of $B_{j, p, \xi}$ by defining $p$ extra knots at each end, and we will assume

$$
\boldsymbol{\xi}:=\left\{\xi_{j-p} \leq \cdots \leq \xi_{j-1}<\xi_{j} \leq \cdots \leq \xi_{j+p+1}<\xi_{j+p+2} \leq \cdots \leq \xi_{j+2 p+1}\right\} .
$$

These extra knots can be defined in any way we like. One possibility is

$$
\xi_{j-p}=\cdots=\xi_{j-1}:=\xi_{j}-1, \quad \xi_{j+p+1}+1=: \xi_{j+p+2}=\cdots=\xi_{j+2 p+1} .
$$

On such a knot sequence $2 p+1$ B-splines $B_{i, p, \boldsymbol{\xi}}=B\left[\xi_{i}, \ldots, \xi_{i+p+1}\right], i=j-$ $p, \ldots, j+p$ are well defined.

The following identity was first proved by Marsden [36] and simplifies many dealings with B-splines.

Theorem 8 (Local Marsden Identity). For $j \leq m \leq j+p$ and $\xi_{m}<\xi_{m+1}$, we have

$$
(y-x)^{p}=\sum_{i=m-p}^{m} \psi_{i, p, \boldsymbol{\xi}}(y) B_{i, p, \boldsymbol{\xi}}(x), \quad x \in\left[\xi_{m}, \xi_{m+1}\right), \quad y \in \mathbb{R} .
$$

If $B_{i, p, \boldsymbol{\xi}}^{\{m\}}$ is the polynomial which is equal to $B_{i, p, \boldsymbol{\xi}}(x)$ for $x \in\left[\xi_{m}, \xi_{m+1}\right)$ then

$$
(y-x)^{p}=\sum_{i=m-p}^{m} \psi_{i, p, \boldsymbol{\xi}}(y) B_{i, p, \boldsymbol{\xi}}^{\{m\}}(x), \quad x, y \in \mathbb{R} .
$$

Proof. Suppose $x \in\left[\xi_{m}, \xi_{m+1}\right)$. The equality (2.17) can be proved by induction. It is clearly true for $p=0$. Let us now assume it holds for degree $p-1$. Then, by means of the dual recurrence (2.11) and the B-spline recurrence relation we obtain

$$
\begin{aligned}
(y-x)^{p} & =(y-x)(y-x)^{p-1}=(y-x) \sum_{i=m-p+1}^{m} \psi_{i, p-1, \boldsymbol{\xi}}(y) B_{i, p-1, \boldsymbol{\xi}}(x) \\
& =\sum_{i=m-p+1}^{m}\left(\frac{x-\xi_{i}}{\xi_{i+p}-\xi_{i}} \psi_{i, p, \boldsymbol{\xi}}(y)+\frac{\xi_{i+p}-x}{\xi_{i+p}-\xi_{i}} \psi_{i-1, p, \boldsymbol{\xi}}\right) B_{i, p-1, \boldsymbol{\xi}}(x) \\
& =\sum_{i=m-p}^{m}\left(\frac{x-\xi_{i}}{\xi_{i+p}-\xi_{i}} B_{i, p-1, \boldsymbol{\xi}}(x)+\frac{\xi_{i+p+1}-x}{\xi_{i+p+1}-\xi_{i+1}} B_{i+1, p-1, \boldsymbol{\xi}}(x)\right) \psi_{i, p, \boldsymbol{\xi}}(y) \\
& =\sum_{i=m-p}^{m} \psi_{i, p, \boldsymbol{\xi}}(y) B_{i, p, \boldsymbol{\xi}}(x) .
\end{aligned}
$$

Here we used that $\frac{x-\xi_{i}}{\xi_{i+p}-\xi_{i}} B_{i, p-1, \xi}(x)=0$ for $i=m-p, m+1$.

The local Marsden identity immediately leads to the following properties, where we suppose $\xi_{m}<\xi_{m+1}$ for some $j \leq m \leq j+p$. 
- Local Representation of Monomials. We have for $p \geq k$,

$$
x^{k}=\sum_{i=m-p}^{m}\left((-1)^{k} \frac{k !}{p !} D^{p-k} \psi_{j, p, \boldsymbol{\xi}}(0)\right) B_{i, p, \boldsymbol{\xi}}(x), \quad x \in\left[\xi_{m}, \xi_{m+1}\right) .
$$

Proof. Fix $x \in\left[\xi_{m}, \xi_{m+1}\right)$. Differentiating $p-k$ times with respect to $y$ in (2.18) results in

$$
\frac{(y-x)^{k}}{k !}=\sum_{i=m-p}^{m}\left(\frac{1}{p !} D^{p-k} \psi_{i, p, \boldsymbol{\xi}}(y)\right) B_{i, p, \boldsymbol{\xi}}(x), \quad y \in \mathbb{R},
$$

for $k=0,1, \ldots, p$. Setting $y=0$ in (2.20) results in (2.19).

- Local Partition of Unity. Taking $k=0$ in (2.19) gives

$$
\sum_{i=m-p}^{m} B_{i, p, \boldsymbol{\xi}}(x)=1, \quad x \in\left[\xi_{m}, \xi_{m+1}\right) .
$$

- Local Linear Independence. The two sets $\left\{B_{i, p, \xi}\right\}_{i=m-p}^{m}$ and $\left\{\psi_{i, p, \boldsymbol{\xi}}\right\}_{i=m-p}^{m}$ form both a basis for the polynomial space $\mathbb{P}_{p}$ on any subset of $\left[\xi_{m}, \xi_{m+1}\right)$ containing at least $p+1$ distinct points.

Proof. Let $A$ be a subset of $\left[\xi_{m}, \xi_{m+1}\right)$ containing at least $p+1$ distinct points. From (2.20) we see that on $A$ every polynomial of degree at most $p$ can be written as a linear combination of the $p+1$ polynomials $B_{i, p, \boldsymbol{\xi}}^{\{m\}}, i=m-p, \ldots, m$. Since the dimension of the space $\mathbb{P}_{p}$ on $A$ is $p+1$, these polynomials must be linearly independent and a basis. The result for $\left\{\psi_{i, p, \boldsymbol{\xi}}\right\}_{i=m-p}^{m}$ follows by symmetry.

\subsection{Smoothness, Differentiation and Integration}

The derivative of a B-spline can be expressed by means of a simple difference formula.

Theorem 9 (Differentiation). We have

$$
D_{+} B_{j, p, \boldsymbol{\xi}}(x)=p\left(\frac{B_{j, p-1, \boldsymbol{\xi}}(x)}{\xi_{j+p}-\xi_{j}}-\frac{B_{j+1, p-1, \boldsymbol{\xi}}(x)}{\xi_{j+p+1}-\xi_{j+1}}\right), \quad p \geq 1,
$$

where fractions with zero denominator have value zero.

Proof. If $\xi_{j+p+1}=\xi_{j}$ then both sides of (2.22) are zero, so we can assume $\xi_{j+p+1}>$ $\xi_{j}$. We continue to use the extra knots (2.15). If $x<\xi_{j}$ or $x \geq \xi_{j+p+1}$ then both sides of (2.22) are zero. Otherwise $x \in\left[\xi_{m}, \xi_{m+1}\right)$ for some $m$ with $j \leq m \leq j+p$ and it is enough to prove (2.22) for such an interval. Differentiating both sides of (2.17) with respect to $x$ gives 


$$
-p(y-x)^{p-1}=\sum_{i=m-p}^{m} D B_{i, p, \boldsymbol{\xi}}(x) \psi_{i, p}(y), \quad x \in\left[\xi_{m}, \xi_{m+1}\right) .
$$

On the other hand, using the local Marsden identity (2.17) for degree $p-1$ and the difference formula for dual polynomials (2.12) results in

$$
\begin{aligned}
-p(y-x)^{p-1} & =-p \sum_{i=m-p+1}^{m} \psi_{i, p-1}(y) B_{i, p-1, \boldsymbol{\xi}}(x) \\
& =p \sum_{i=m-p+1}^{m}\left(\frac{\psi_{i, p}(y)}{\xi_{i+p}-\xi_{i}}-\frac{\psi_{i-1, p}(y)}{\xi_{i+p}-\xi_{i}}\right) B_{i, p-1, \boldsymbol{\xi}}(x) \\
& =\sum_{i=m-p}^{m} p\left(\frac{B_{i, p-1, \xi}(x)}{\xi_{i+p}-\xi_{i}}-\frac{B_{i+1, p-1, \xi}(x)}{\xi_{i+p+1}-\xi_{i+1}}\right) \psi_{i, p}(y) .
\end{aligned}
$$

When comparing this with (2.23) and using the linear independence of the dual polynomials, it follows that (2.22) holds for $i=m-p, \ldots, m$. In particular, since $m-p \leq j \leq m$, (2.22) holds for $i=j$.

Example 10. The differentiation formula (2.22) for $p=2$ together with the expression (2.2) immediately gives the piecewise form of the derivative of the quadratic B-spline $B_{j, 2, \xi}$ :

$$
D_{+} B_{j, 2, \boldsymbol{\xi}}(x)= \begin{cases}\frac{2\left(x-\xi_{j}\right)}{\left(\xi_{j+2}-\xi_{j}\right)\left(\xi_{j+1}-\xi_{j}\right)}, & \text { if } x \in\left[\xi_{j}, \xi_{j+1}\right), \\ \frac{2\left(\xi_{j+2}-x\right)}{\left(\xi_{j+2}-\xi_{j}\right)\left(\xi_{j+2}-\xi_{j+1}\right)}-\frac{2\left(x-\xi_{j+1}\right)}{\left(\xi_{j+3}-\xi_{j+1}\right)\left(\xi_{j+2}-\xi_{j+1}\right)}, & \text { if } x \in\left[\xi_{j+1}, \xi_{j+2}\right), \\ -\frac{2\left(\xi_{j+3}-x\right)}{\left(\xi_{j+3}-\xi_{j+1}\right)\left(\xi_{j+3}-\xi_{j+2}\right)}, & \text { if } x \in\left[\xi_{j+2}, \xi_{j+3}\right), \\ 0, & \text { otherwise. }\end{cases}
$$

This is in agreement with taking the derivative of the piecewise expression (2.3) of $B_{j, 2, \xi}$ given in Example 4.

Proposition 11. The $r$-th derivative of the $B$-spline $B_{j, p, \xi}$ for $0 \leq r \leq p$ can be bounded as follows. For any $x \in\left[\xi_{m}, \xi_{m+1}\right)$ with $j \leq m \leq j+p$ we have

$$
\left|D^{r} B_{j, p, \boldsymbol{\xi}}(x)\right| \leq 2^{r} \frac{p !}{(p-r) !} \prod_{k=p-r+1}^{p} \frac{1}{\Delta_{m, k}},
$$

where

$$
\Delta_{m, k}:=\min _{m-k+1 \leq i \leq m} h_{i, k}, \quad h_{i, k}:=\xi_{i+k}-\xi_{i}, \quad k=1, \ldots, p .
$$

Proof. This holds for $r=0$ because of the nonnegativity of $B_{j, p, \xi}$ and the partition of unity property (2.21). By the differentiation formula (2.22) and the local support property (2.6) we have 


$$
\begin{aligned}
& D^{r} B_{j, p, \boldsymbol{\xi}}(x) \\
& =p \begin{cases}-D^{r-1} B_{j+1, p-1, \boldsymbol{\xi}}(x) / h_{j+1, p}, & \text { if } m=j+p, \\
D^{r-1} B_{j, p-1, \boldsymbol{\xi}}(x) / h_{j, p}-D^{r-1} B_{j+1, p-1, \boldsymbol{\xi}}(x) / h_{j+1, p}, & \text { if } j<m<j+p, \\
D^{r-1} B_{j, p-1, \boldsymbol{\xi}}(x) / h_{j, p}, & \text { if } m=j .\end{cases}
\end{aligned}
$$

It follows that

$$
\left|D^{r} B_{j, p, \boldsymbol{\xi}}(x)\right| \leq 2 p \max _{m-p+1 \leq i \leq m}\left|D^{r-1} B_{i, p-1, \boldsymbol{\xi}}(x)\right| / \Delta_{m, p}
$$

and by induction on $r$ we obtain (2.24).

Note that the upper bound in (2.24) is well defined since $\Delta_{m, k} \geq \xi_{m+1}-\xi_{m}>0$.

Theorem 12 (Smoothness). If $\xi$ is a knot of $B_{j, p, \xi}$ of multiplicity $\mu \leq p+1$, then

$$
B_{j, p, \xi} \in C^{p-\mu}(\xi)
$$

i.e., its derivatives of order $0,1, \ldots, p-\mu$ are continuous at $\xi$.

Proof. Suppose $\xi$ is a knot of $B_{j, p, \xi}$ of multiplicity $\mu$. We first consider the smoothness property when $\mu=p+1$. For $x \in\left[\xi_{j}, \xi_{j+p+1}\right)$ it follows immediately from (2.4) and (2.5) that

$$
\begin{aligned}
& B_{j, p, \boldsymbol{\xi}}(x)=\left(x-\xi_{j}\right)^{p} /\left(\xi_{j+p+1}-\xi_{j}\right)^{p}, \quad \xi_{j}<\xi_{j+1}=\cdots=\xi_{j+p+1}, \\
& B_{j, p, \boldsymbol{\xi}}(x)=\left(\xi_{j+p+1}-x\right)^{p} /\left(\xi_{j+p+1}-\xi_{j}\right)^{p}, \quad \xi_{j}=\cdots=\xi_{j+p}<\xi_{j+p+1} .
\end{aligned}
$$

These two B-splines are discontinuous with a jump of absolute size one at the multiple knot showing the smoothness property for $\mu=p+1$.

Let us now consider the case where $B_{j, p, \boldsymbol{\xi}}$ has an interior knot of multiplicity equal to $\mu=p$, i.e., $\xi_{j}<\xi_{j+1}=\cdots=\xi_{j+p}<\xi_{j+p+1}$. For $x \in\left[\xi_{j}, \xi_{j+p+1}\right)$ it follows from (2.4) and (2.5) that

$$
B_{j, p, \boldsymbol{\xi}}(x)=\frac{\left(x-\xi_{j}\right)^{p}}{\left(\xi_{j+p}-\xi_{j}\right)^{p}} B_{j, 0, \boldsymbol{\xi}}(x)+\frac{\left(\xi_{j+p+1}-x\right)^{p}}{\left(\xi_{j+p+1}-\xi_{j+1}\right)^{p}} B_{j+p, 0, \boldsymbol{\xi}}(x) .
$$

The two nontrivial pieces have both value one at the center knot $\xi_{j+1}=\xi_{j+p}$, and $B_{j, p, \boldsymbol{\xi}}$ is continuous on $\mathbb{R}$. Moreover, the first derivative has a nonzero jump at the center knot.

For the remaining cases we use induction on $p$ to show that $B_{j, p, \xi} \in C^{p-\mu}(\xi)$. The case $p=1$ follows from Example 3. Suppose for some $p \geq 2$ that $B_{j, p-1, \xi} \in$ $C^{p-1-\mu}(\xi)$ at a knot $\xi$ of multiplicity $\mu$. For the multiplicity $p$ case $\xi=\xi_{j}=\cdots=$ $\xi_{j+p-1}<\xi_{j+p} \leq \xi_{j+p+1}$ we use the recurrence relation

$$
B_{j, p, \boldsymbol{\xi}}(x)=\frac{x-\xi_{j}}{\xi_{j+p}-\xi_{j}} B_{j, p-1, \boldsymbol{\xi}}(x)+\frac{\xi_{j+p+1}-x}{\xi_{j+p+1}-\xi_{j+1}} B_{j+1, p-1, \xi}(x) .
$$


The first term vanishes at $x=\xi=\xi_{j}$. Since $B_{j+1, p-1, \xi}$ has a knot of multiplicity $p-1$ at $\xi$, it follows from the induction hypothesis that it is continuous there. We conclude that $B_{j, p, \xi}$ is continuous at $\xi$. The case where the right end knot of $B_{j, p, \boldsymbol{\xi}}$ has multiplicity $p$ is handled similarly. Finally, if $\mu \leq p-1$ then both terms in the differentiation formula (2.22) has a knot of multiplicity at most $\mu$ at $\xi$ and by the induction hypothesis we obtain $D_{+} B_{j, p, \xi} \in C^{p-1-\mu}(\xi)$. Moreover, by the recurrence relation and the induction hypothesis it follows that $B_{j, p, \xi}$ is continuous at $\xi$, and so we also conclude that $B_{j, p, \xi} \in C^{p-\mu}(\xi)$ if $\mu \leq p-1$. This completes the proof.

The B-spline $B_{j, p, \xi}$ is supported on the interval $\left[\xi_{j}, \xi_{j+p+1}\right]$. Hence, Theorem 12 implies that $B_{j, p, \xi}$ is continuous on $\mathbb{R}$ whenever $\xi_{j+p}>\xi_{j}$ and $\xi_{j+p+1}>\xi_{j+1}$. Similarly, $B_{j, p, \xi}$ is $C^{r}$-continuous on $\mathbb{R}$ whenever $\xi_{j+p-r+i}>\xi_{j+i}$ for each $i=0, \ldots, r+1$ and $-1 \leq r<p$.

Theorem 13 (Integration). We have

$$
\gamma_{j, p, \boldsymbol{\xi}}:=\int_{\xi_{j}}^{\xi_{j+p+1}} B_{j, p, \boldsymbol{\xi}}(x) \mathrm{d} x=\frac{\xi_{j+p+1}-\xi_{j}}{p+1} .
$$

Proof. This time we define $p+1$ extra knots at each end, and we assume

$$
\boldsymbol{\xi}:=\left\{\xi_{j-p-1}=\cdots=\xi_{j-1}<\xi_{j} \leq \cdots \leq \xi_{j+p+1}<\xi_{j+p+2}=\cdots=\xi_{j+2 p+2}\right\} .
$$

On this knot sequence we consider $p+1 \mathrm{~B}$-splines $B_{i, p+1, \boldsymbol{\xi}}, i=j-p-1, \ldots, j-1$ of degree $p+1$. From Theorem 12 we know that these B-splines are continuous on $\mathbb{R}$. Therefore, we get for $i=j-p-1, \ldots, j-1$,

$$
0=B_{i, p+1, \boldsymbol{\xi}}\left(\xi_{i+p+2}\right)-B_{i, p+1, \boldsymbol{\xi}}\left(\xi_{i}\right)=\int_{\xi_{i}}^{\xi_{i+p+2}} D_{+} B_{i, p+1, \boldsymbol{\xi}}(x) \mathrm{d} x=E_{i}-E_{i+1},
$$

where by the local support and the differentiation formula (2.22),

$$
E_{i}:=\frac{p+1}{\xi_{i+p+1}-\xi_{i}} \int_{\xi_{i}}^{\xi_{i+p+1}} B_{i, p, \boldsymbol{\xi}}(x) \mathrm{d} x, \quad i=j-p-1, \ldots, j .
$$

This means that $E_{j}=E_{j-1}=\cdots=E_{j-p-1}$. Moreover, since $\xi_{j-p-1}=\cdots=\xi_{j-1}$, we obtain from (2.28) that

$$
E_{j-p-1}=\frac{p+1}{\xi_{j}-\xi_{j-p-1}} \int_{\xi_{j-p-1}}^{\xi_{j}} \frac{\left(\xi_{j}-x\right)^{p}}{\left(\xi_{j}-\xi_{j-p-1}\right)^{p}} \mathrm{~d} x=1,
$$

and the integration formula (2.30) follows. 


\section{Splines}

A spline function (in short, spline) is a linear combination of B-splines defined on a given knot sequence with a fixed degree. In this section we analyze the space of splines and discuss several of their properties.

\subsection{The Spline Space $\mathbb{S}_{p, \xi}$ and Some Spline Properties}

Suppose for integers $n>p \geq 0$ that a knot sequence

$$
\boldsymbol{\xi}:=\left\{\xi_{i}\right\}_{i=1}^{n+p+1}=\left\{\xi_{1} \leq \xi_{2} \leq \cdots \leq \xi_{n+p+1}\right\}, \quad n \in \mathbb{N}, \quad p \in \mathbb{N}_{0}
$$

is given. This knot sequence allows us to define a set of $n$ B-splines of degree $p$, namely

$$
\left\{B_{1, p, \xi}, \ldots, B_{n, p, \xi}\right\}
$$

We consider the space

$$
\mathbb{S}_{p, \boldsymbol{\xi}}:=\left\{s:\left[\xi_{p+1}, \xi_{n+1}\right] \rightarrow \mathbb{R}: s=\sum_{j=1}^{n} c_{j} B_{j, p, \xi}, c_{j} \in \mathbb{R}\right\} .
$$

This is the space of splines spanned by the B-splines in (3.1) over the interval $\left[\xi_{p+1}, \xi_{n+1}\right]$, which is called the basic interval.

We now introduce some terminology to identify certain properties of knot sequences which are crucial in the study of the space (3.2).

- A knot sequence $\xi$ is called $(p+1)$-regular if $\xi_{j}<\xi_{j+p+1}$ for $j=1, \ldots, n$. By the local support (2.6) such a knot sequence ensures that all the B-splines in (3.1) are not identically zero.

- A knot sequence $\boldsymbol{\xi}$ is called $(p+1)$-basic if it is $(p+1)$-regular with $\xi_{p+1}<\xi_{p+2}$ and $\xi_{n}<\xi_{n+1}$. As we will show later, the B-splines in (3.1) defined on a $(p+1)$ basic knot sequence are linearly independent on the basic interval $\left[\xi_{p+1}, \xi_{n+1}\right]$.

- A knot sequence $\boldsymbol{\xi}$ is called $(p+1)$-open on an interval $[a, b]$ if it is $(p+1)$ regular and it has end knots of multiplicity $p+1$, i.e.,

$$
a:=\xi_{1}=\cdots=\xi_{p+1}<\xi_{p+2} \leq \cdots \leq \xi_{n}<\xi_{n+1}=\cdots=\xi_{n+p+1}=: b .
$$

This sequence is often used in practice. In particular, it turns out to be natural to construct open curves, clamped at two given points. Note that $(p+1)$-open implies $(p+1)$-basic.

Some further preliminary remarks are in order here.

- We consider B-splines on a closed basic interval $\left[\xi_{p+1}, \xi_{n+1}\right]$. In order to avoid the asymmetry at the right endpoint we define the B-splines to be left continuous 




Fig. 2 The B-spline basis of degree $p=3$ on a $(p+1)$-open knot sequence. The knot positions are visualized by vertical dotted lines.

at the right endpoint, i.e., its value at $\xi_{n+1}$ is obtained by taking limits from the left:

$$
B_{j, p, \boldsymbol{\xi}}\left(\xi_{n+1}\right):=\lim _{\substack{x \rightarrow \xi_{n+1} \\ x<\xi_{n+1}}} B_{j, p, \boldsymbol{\xi}}(x), \quad j=1, \ldots, n .
$$

Note that for a $(p+1)$-open knot sequence the end condition (3.4) means that $B_{n, p, \boldsymbol{\xi}}\left(\xi_{n+p+1}\right)=1$ and (2.6) has to be modified for this B-spline.

- We define a multiplicity function $\mu_{\boldsymbol{\xi}}: \mathbb{R} \rightarrow \mathbb{N}_{0}$ given by $\mu_{\boldsymbol{\xi}}\left(\xi_{i}\right)=\mu_{i}$ if $\xi_{i} \in \boldsymbol{\xi}$ occurs exactly $\mu_{i} \geq 1$ times in $\boldsymbol{\xi}$, and $\mu_{\boldsymbol{\xi}}(x)=0$ if $x \notin \boldsymbol{\xi}$. If $\boldsymbol{\xi}$ and $\tilde{\xi}$ are two knot sequences we say that $\boldsymbol{\xi} \subseteq \tilde{\boldsymbol{\xi}}$ if $\mu_{\xi}(x) \leq \mu_{\tilde{\xi}}(x)$ for all $x \in \mathbb{R}$.

- Without loss of generality, we can always assume that the end knots have multiplicity $p+1$. If this is not the case, then we can add extra knots at the ends and assume the extra B-splines to have coefficients zero. This observation simplifies many proofs.

Example 14. Figure 2 illustrates all the B-splines of degree $p=3$ on a $(p+1)$-open knot sequence, where the interior knots are simple.

From the properties of B-splines, we immediately conclude the following properties of the spline representation in (3.2).

- Smoothness. If $\xi$ is a knot of multiplicity $\mu$ then $s \in C^{r}(\xi)$ for any $s \in \mathbb{S}_{p, \xi}$, where $r+\mu=p$. This follows from the smoothness property of the B-splines (Theorem 12). The relation between smoothness, multiplicity and degree is as follows:

$$
\text { "smoothness }+ \text { multiplicity }=\text { degree". }
$$

- Local Support. The local support (2.6) of the B-splines implies

$$
\sum_{j=1}^{n} c_{j} B_{j, p, \boldsymbol{\xi}}(x)=\sum_{j=m-p}^{m} c_{j} B_{j, p, \boldsymbol{\xi}}(x), \quad x \in\left[\xi_{m}, \xi_{m+1}\right), \quad p+1 \leq m \leq n,
$$

and if $\xi_{m}<\xi_{m+p}$ then 


$$
\sum_{j=1}^{n} c_{j} B_{j, p, \boldsymbol{\xi}}\left(\xi_{m}\right)=\sum_{j=m-p}^{m-1} c_{j} B_{j, p, \boldsymbol{\xi}}\left(\xi_{m}\right), \quad p+1 \leq m \leq n+1
$$

- Minimal Support. From the smoothness properties it can be proved that if the support of $s \in \mathbb{S}_{p, \xi}$ is a proper subset of $\left[\xi_{j}, \xi_{j+p+1}\right]$ for some $j$ then $s=0$. Therefore, the B-splines have minimal support.

- Coefficient Recurrence. For $x \in\left[\xi_{p+1}, \xi_{n+1}\right]$, by the recurrence relation (2.1) we have

$$
\sum_{j=1}^{n} c_{j} B_{j, p, \boldsymbol{\xi}}(x)=\sum_{j=2}^{n} \check{c}_{j}(x) B_{j, p-1, \boldsymbol{\xi}}(x),
$$

where

$$
\check{c}_{j}(x):=\frac{x-\xi_{j}}{\xi_{j+p}-\xi_{j}} c_{j}+\frac{\xi_{j+p}-x}{\xi_{j+p}-\xi_{j}} c_{j-1},
$$

and $\check{c}_{j}(x) B_{j, p-1, \xi}(x)=0$ if $\xi_{j+p}=\xi_{j}$.

- Differentiation. By (2.22) we have

$$
D_{+}\left(\sum_{j=1}^{n} c_{j} B_{j, p, \boldsymbol{\xi}}\right)=\sum_{j=2}^{n} c_{j}^{(1)} B_{j, p-1, \boldsymbol{\xi}}, \quad p \geq 1,
$$

where

$$
c_{j}^{(1)}:=p\left(\frac{c_{j}-c_{j-1}}{\xi_{j+p}-\xi_{j}}\right)
$$

and fractions with zero denominator have value zero.

- Linear Independence. If $\boldsymbol{\xi}$ is $(p+1)$-basic, then the B-splines in (3.1) are linearly independent on the basic interval. Thus, the spline space $\mathbb{S}_{p, \boldsymbol{\xi}}$ is a vector space of dimension $n$.

Proof. We must show that if $s(x)=\sum_{j=1}^{n} c_{j} B_{j, p, \xi}(x)=0$ for $x \in\left[\xi_{p+1}, \xi_{n+1}\right]$ then $c_{j}=0$ for all $j$. Let us fix $1 \leq j \leq n$. Since $\boldsymbol{\xi}$ is $(p+1)$-regular, there is an integer $m_{j}$ with $j \leq m_{j} \leq j+p$ such that $\xi_{m_{j}}<\xi_{m_{j}+1}$. Moreover, the assumptions $\xi_{p+1}<\xi_{p+2}$ and $\xi_{n}<\xi_{n+1}$ guarantee that $\left[\xi_{m_{j}}, \xi_{m_{j}+1}\right)$ can be chosen in the basic interval. From the local support property (3.6) we know

$$
s(x)=\sum_{i=m_{j}-p}^{m_{j}} c_{i} B_{i, p, \boldsymbol{\xi}}(x)=0, \quad x \in\left[\xi_{m_{j}}, \xi_{m_{j}+1}\right) .
$$

The local linear independence property (see Section 2.3) implies $c_{m_{j}-p}=\cdots=$ $c_{m_{j}}=0$, and in particular $c_{j}=0$. 


\subsection{The Piecewise Polynomial Space $\mathbb{S}_{p}^{r}(\Delta)$}

We now prove that the spline space $\mathbb{S}_{p, \xi}$ is nothing else than a space of piecewise polynomials of degree $p$ defined by a given sequence of break points and by some prescribed smoothness. The set of knots $\boldsymbol{\xi}$ must be suitably selected according to the break points and the smoothness conditions. Therefore, the B-splines are a basis of such a space of piecewise polynomials.

Let $\Delta$ be a sequence of distinct real numbers,

$$
\Delta:=\left\{\eta_{0}<\eta_{1}<\cdots<\eta_{\ell+1}\right\}
$$

The elements in $\Delta$ are called break points. Moreover, let $\boldsymbol{r}:=\left(r_{1}, \ldots, r_{\ell}\right)$ be a vector of integers such that $-1 \leq r_{i} \leq p$ for $i=1, \ldots, \ell$. The space $\mathbb{S}_{p}^{r}(\Delta)$ of piecewise polynomials of degree $p$ with smoothness $r$ over the partition $\Delta$ is defined by

$$
\begin{gathered}
\mathbb{S}_{p}^{r}(\Delta):=\left\{s:\left[\eta_{0}, \eta_{\ell+1}\right] \rightarrow \mathbb{R}: s \in \mathbb{P}_{p}\left(\left[\eta_{i}, \eta_{i+1}\right)\right), i=0, \ldots, \ell-1,\right. \\
\left.s \in \mathbb{P}_{p}\left(\left[\eta_{\ell}, \eta_{\ell+1}\right]\right), \quad s \in C^{r_{i}}\left(\eta_{i}\right), i=1, \ldots, \ell\right\} .
\end{gathered}
$$

Suppose that $s^{\{i\}} \in \mathbb{P}_{p}$ is the polynomial equal to the restriction of a given function $s \in \mathbb{S}_{p}^{r}(\Delta)$ to the interval $\left[\eta_{i}, \eta_{i+1}\right), i=0, \ldots, \ell$. Since $s \in C^{r_{i}}\left(\eta_{i}\right)$, we have

$$
s^{\{i\}}(x)-s^{\{i-1\}}(x)=\sum_{j=r_{i}+1}^{p} c_{i, j}\left(x-\eta_{i}\right)^{j}
$$

for some coefficients $c_{i, j}$. It follows that $\mathbb{S}_{p}^{r}(\Delta)$ is spanned by the set of functions

$$
\left\{1, x, \ldots, x^{p},\left(x-\eta_{1}\right)_{+}^{r_{1}+1}, \ldots,\left(x-\eta_{1}\right)_{+}^{p}, \ldots,\left(x-\eta_{\ell}\right)_{+}^{r_{\ell}+1}, \ldots,\left(x-\eta_{\ell}\right)_{+}^{p}\right\},
$$

where the truncated power function $(\cdot)_{+}^{p}$ is defined by

$$
(x)_{+}^{p}:= \begin{cases}x^{p}, & x>0 \\ 0, & x<0\end{cases}
$$

and the value at zero is defined by taking the right limit.

It is easy to see that the functions in (3.13) are linearly independent. Indeed, let

$$
s(x):=\sum_{j=0}^{p} c_{0, j} x^{j}+\sum_{i=1}^{\ell} \sum_{j=r_{i}+1}^{p} c_{i, j}\left(x-\eta_{i}\right)_{+}^{j}=0, \quad x \in\left[\eta_{0}, \eta_{\ell+1}\right] .
$$

On $\left[\eta_{0}, \eta_{1}\right)$ we have $s(x)=\sum_{j=0}^{p} c_{0, j} x^{j}$ and it follows that $c_{0,0}=\cdots=c_{0, p}=0$. Suppose for some $1 \leq k \leq \ell$ that $c_{i, j}=0$ for $i<k$. Then, on $\left[\eta_{k}, \eta_{k+1}\right)$ we have $s(x)=\sum_{j=r_{k}+1}^{p} c_{k, j}\left(x-\eta_{k}\right)^{j}=0$ showing that all $c_{k, j}=0$.

This implies that the set of functions in (3.13) is a basis for $\mathbb{S}_{p}^{r}(\Delta)$, the so-called truncated power basis. As a consequence, 


$$
\operatorname{dim}\left(\mathbb{S}_{p}^{r}(\Delta)\right)=p+1+\sum_{i=1}^{\ell}\left(p-r_{i}\right)
$$

The next theorem shows that the set of B-splines in (3.1) defined over a specific knot sequence $\boldsymbol{\xi}$ forms an alternative basis for $\mathbb{S}_{p}^{r}(\Delta)$. This was first proved by Curry and Schoenberg in [16].

Theorem 15 (Characterization of Spline Space). The piecewise polynomial space $\mathbb{S}_{p}^{r}(\Delta)$ is characterized in terms of $B$-splines by

$$
\mathbb{S}_{p}^{r}(\Delta)=\mathbb{S}_{p, \boldsymbol{\xi}}
$$

where the knot sequence $\boldsymbol{\xi}:=\left\{\xi_{i}\right\}_{i=1}^{n+p+1}$ with $n:=\operatorname{dim}\left(\mathbb{S}_{p}^{r}(\Delta)\right)$ is constructed such that

$$
\xi_{1} \leq \cdots \leq \xi_{p+1}:=\eta_{0}, \quad \eta_{\ell+1}=: \xi_{n+1} \leq \cdots \leq \xi_{n+p+1},
$$

and

$$
\xi_{p+2}, \ldots, \xi_{n}:=\overbrace{\eta_{1}, \ldots, \eta_{1}}^{p-r_{1}}, \ldots, \overbrace{\eta_{\ell}, \ldots, \eta_{\ell}}^{p-r_{\ell}} .
$$

Proof. From the piecewise polynomial and smoothness properties of B-splines it follows that the B-spline space $\mathbb{S}_{p, \xi}$ is a subspace of $\mathbb{S}_{p}^{r}(\Delta)$. Moreover, the constructed knot sequence $\boldsymbol{\xi}$ is $(p+1)$-basic, so $\operatorname{dim}\left(\mathbb{S}_{p, \boldsymbol{\xi}}\right)=n$ by the linear independence property of B-splines. This implies that $\mathbb{S}_{p}^{r}(\Delta)=\mathbb{S}_{p, \boldsymbol{\xi}}$.

Example 16. Consider $\Delta:=\left\{\eta_{0}<\eta_{1}<\eta_{2}<\eta_{3}\right\}$ and the space $\mathbb{S}_{3}^{r}(\Delta)$ with $\boldsymbol{r}=\left(r_{1}, r_{2}\right)=(2,1)$. It follows from Theorem 15 that $\mathbb{S}_{3}^{r}(\Delta)=\mathbb{S}_{3, \xi}$, where

$$
\boldsymbol{\xi}=\left\{\xi_{i}\right\}_{i=1}^{7+3+1}=\left\{\eta_{0}=\eta_{0}=\eta_{0}=\eta_{0}<\eta_{1}<\eta_{2}=\eta_{2}<\eta_{3}=\eta_{3}=\eta_{3}=\eta_{3}\right\} .
$$

This knot sequence is 4-open.

Finally, we give a characterization for the space spanned by the $r$-th derivatives of B-splines for $0 \leq r \leq p$, i.e.,

$$
D_{+}^{r} \mathbb{S}_{p, \boldsymbol{\xi}}:=\left\{s:\left[\xi_{p+1}, \xi_{n+1}\right] \rightarrow \mathbb{R}: s=D_{+}^{r}\left(\sum_{j=1}^{n} c_{j} B_{j, p, \boldsymbol{\xi}}\right), c_{j} \in \mathbb{R}\right\} .
$$

Theorem 17 (Characterization of Derivative Spline Space). Given a knot sequence $\boldsymbol{\xi}:=\left\{\xi_{i}\right\}_{i=1}^{n+p+1}$, we have for $0 \leq r \leq p$,

$$
D_{+}^{r} \mathbb{S}_{p, \boldsymbol{\xi}}=\mathbb{S}_{p-r, \boldsymbol{\xi}_{r}},
$$

where $\boldsymbol{\xi}_{r}:=\left\{\xi_{i}\right\}_{i=r+1}^{n+p+1-r}$.

Proof. The result is obvious for $r=0$. Let us now consider the case $r=1$, for which we note that

$$
\left\{B_{1, p-1, \boldsymbol{\xi}_{1}}, \ldots, B_{n-1, p-1, \boldsymbol{\xi}_{1}}\right\}=\left\{B_{2, p-1, \boldsymbol{\xi}}, \ldots, B_{n, p-1, \boldsymbol{\xi}}\right\} .
$$


By the differentiation formula (3.10) it is clear that

$$
D_{+}\left(\sum_{j=1}^{n} c_{j} B_{j, p, \boldsymbol{\xi}}\right)=p \sum_{j=2}^{n}\left(\frac{c_{j}-c_{j-1}}{\xi_{j+p}-\xi_{j}}\right) B_{j, p-1, \boldsymbol{\xi}} \in \mathbb{S}_{p-1, \boldsymbol{\xi}_{1}} .
$$

On the other hand, suppose $s \in \mathbb{S}_{p-1, \boldsymbol{\xi}_{1}}$, represented as $s=\sum_{j=2}^{n} d_{j} B_{j, p-1, \boldsymbol{\xi}}$. Then, by using again the differentiation formula, we can write $s=D_{+}\left(\sum_{j=1}^{n} c_{j} B_{j, p, \xi}\right)$, where $c_{1}$ can be any real number and

$$
c_{j}=c_{j-1}+\frac{\xi_{j+p}-\xi_{j}}{p} d_{j}, \quad j=2, \ldots, n .
$$

For $r>1$ we use the relation $D_{+}^{r}=D_{+} D_{+}^{r-1}$.

By combining Theorem 15 and Theorem 17 it follows that for $0 \leq r \leq p$,

$$
\mathbb{S}_{p-r}^{r-r}(\Delta)=D_{+}^{r} \mathbb{S}_{p, \boldsymbol{\xi}}
$$

where $\boldsymbol{r}-r:=\left(\max \left(r_{1}-r,-1\right), \ldots, \max \left(r_{\ell}-r,-1\right)\right)$ and the knot sequence $\boldsymbol{\xi}$ is constructed as in Theorem 15 .

\subsection{B-Spline Representation of Polynomials}

Polynomials can be represented in terms of B-splines of at least the same degree. We now derive an explicit expression for their B-spline coefficients by using the dual polynomials and the (local) Marsden identity.

Theorem 18 (Marsden Identity). We have

$$
(y-x)^{p}=\sum_{j=1}^{n} \psi_{j, p, \boldsymbol{\xi}}(y) B_{j, p, \boldsymbol{\xi}}(x), \quad x \in\left[\xi_{p+1}, \xi_{n+1}\right], \quad y \in \mathbb{R},
$$

where $\psi_{j, p, \boldsymbol{\xi}}(y):=\left(y-\xi_{j+1}\right) \cdots\left(y-\xi_{j+p}\right)$ is the polynomial of degree $p$ that is dual to $B_{j, p, \boldsymbol{\xi}}$.

Proof. This follows immediately from the local version (2.17). Indeed, if $x \in$ $\left[\xi_{p+1}, \xi_{n+1}\right)$ then $x \in\left[\xi_{m}, \xi_{m+1}\right)$ for some $p+1 \leq m \leq n$, and by the local support property (3.6) we get

$$
(y-x)^{p}=\sum_{j=m-p}^{m} \psi_{j, p, \boldsymbol{\xi}}(y) B_{j, p, \boldsymbol{\xi}}(x)=\sum_{j=1}^{n} \psi_{j, p, \boldsymbol{\xi}}(y) B_{j, p, \boldsymbol{\xi}}(x) .
$$

Taking into account the left continuity of B-splines at the endpoint $\xi_{n+1}$, see (3.4), we arrive at the Marsden identity (3.15). 
Differentiating $p-k$ times with respect to $y$ in (3.15) results in the following formula.

Corollary 19. For $k=0,1, \ldots, p$ we have

$$
\frac{(y-x)^{k}}{k !}=\sum_{j=1}^{n}\left(\frac{1}{p !} D^{p-k} \psi_{j, p, \boldsymbol{\xi}}(y)\right) B_{j, p, \boldsymbol{\xi}}(x), \quad x \in\left[\xi_{p+1}, \xi_{n+1}\right], \quad y \in \mathbb{R} .
$$

Corollary 19 immediately leads to the following properties.

- Representation of Monomials. For $k=0,1, \ldots, p$ we have

$$
x^{k}=\sum_{j=1}^{n} \xi_{j, p, \boldsymbol{\xi}}^{*, k} B_{j, p, \boldsymbol{\xi}}(x), \quad x \in\left[\xi_{p+1}, \xi_{n+1}\right]
$$

where

$$
\xi_{j, p, \boldsymbol{\xi}}^{*, k}:=(-1)^{k} \frac{k !}{p !} D^{p-k} \psi_{j, p, \boldsymbol{\xi}}(0) .
$$

This follows from (3.16) with $y=0$.

- Partition of Unity. Taking $k=0$ in (3.17) gives

$$
\sum_{j=1}^{n} B_{j, p, \boldsymbol{\xi}}(x)=1, \quad x \in\left[\xi_{p+1}, \xi_{n+1}\right] .
$$

Since the B-splines are nonnegative it follows that they form a nonnegative partition of unity on $\left[\xi_{p+1}, \xi_{n+1}\right]$.

- Greville Points. Taking $k=1$ in (3.17) gives for $p \geq 1$,

$$
x=\sum_{j=1}^{n} \xi_{j, p, \boldsymbol{\xi}}^{*} B_{j, p, \xi}(x), \quad x \in\left[\xi_{p+1}, \xi_{n+1}\right]
$$

where

$$
\xi_{j, p, \boldsymbol{\xi}}^{*}:=\xi_{j, p, \boldsymbol{\xi}}^{*, 1}=\frac{\xi_{j+1}+\cdots+\xi_{j+p}}{p} .
$$

The number $\xi_{j, p, \boldsymbol{\xi}}^{*}$ is called Greville point ${ }^{3}$. It is also known as knot average or node.

Example 20. For $p=3$ the equation (3.17) gives

\footnotetext{
${ }^{3}$ An explicit expression of (3.21) was given by Greville in [24]. According to Schoenberg [43], Greville reviewed the paper [43] introducing some elegant simplifications.
} 


$$
\begin{aligned}
1 & =\sum_{j=1}^{n} B_{j, 3, \boldsymbol{\xi}}(x), \\
x & =\sum_{j=1}^{n} \frac{\xi_{j+1}+\xi_{j+2}+\xi_{j+3}}{3} B_{j, 3, \boldsymbol{\xi}}(x), \\
x^{2} & =\sum_{j=1}^{n} \frac{\xi_{j+1} \xi_{j+2}+\xi_{j+1} \xi_{j+3}+\xi_{j+2} \xi_{j+3}}{3} B_{j, 3, \boldsymbol{\xi}}(x), \\
x^{3} & =\sum_{j=1}^{n} \xi_{j+1} \xi_{j+2} \xi_{j+3} B_{j, 3, \boldsymbol{\xi}}(x) .
\end{aligned}
$$

We finally present an expression for the B-spline coefficients of a general polynomial.

Proposition 21 (Representation of Polynomials). Any polynomial $g$ of degree $p$ can be represented as

$$
g(x)=\sum_{j=1}^{n} \Lambda_{j, p, \boldsymbol{\xi}}(g) B_{j, p, \boldsymbol{\xi}}(x), \quad x \in\left[\xi_{p+1}, \xi_{n+1}\right]
$$

where

$$
\Lambda_{j, p, \boldsymbol{\xi}}(g):=\frac{1}{p !} \sum_{r=0}^{p}(-1)^{p-r} D^{r} \psi_{j, p, \boldsymbol{\xi}}\left(\tau_{j}\right) D^{p-r} g\left(\tau_{j}\right), \quad \tau_{j} \in \mathbb{R} .
$$

Proof. The polynomial $g$ can be represented in Taylor form (5.5) as

$$
g(x)=\sum_{r=0}^{p} \frac{\left(x-\tau_{j}\right)^{p-r}}{(p-r) !} D^{p-r} g\left(\tau_{j}\right), \quad \tau_{j} \in \mathbb{R} .
$$

The result follows when we apply (3.16) with $k=p-r$.

Note that, if $\tau_{j}$ is a root of $\psi_{j}$ of multiplicity $\mu_{j}$ then $D^{r} \psi_{i}\left(\tau_{j}\right)=0, r=$ $0,1, \ldots, \mu_{j}-1$ and (3.23) becomes

$$
\Lambda_{j, p, \boldsymbol{\xi}}(g)=\frac{1}{p !} \sum_{r=\mu_{j}}^{p}(-1)^{p-r} D^{r} \psi_{j, p, \boldsymbol{\xi}}\left(\tau_{j}\right) D^{p-r} g\left(\tau_{j}\right), \quad \tau_{j} \in \mathbb{R} .
$$

Example 22. The polynomial $g(x)=a x^{2}+b x+c$ can be represented in terms of quadratic Bsplines:

$$
a x^{2}+b x+c=\sum_{j=1}^{n} c_{j} B_{j, 2, \xi}(x)
$$

From (3.22)-(3.23) with $\psi_{j, 2, \boldsymbol{\xi}}(y):=\left(y-\xi_{j+1}\right)\left(y-\xi_{j+2}\right)$, we obtain that

$$
\begin{aligned}
c_{j}=\Lambda_{j, 2, \boldsymbol{\xi}}(g) & =\frac{1}{2}\left[\left(\tau_{j}-\xi_{j+1}\right)\left(\tau_{j}-\xi_{j+2}\right) 2 a-\left(2 \tau_{j}-\xi_{j+1}-\xi_{j+2}\right)\left(2 a \tau_{j}+b\right)+2\left(a \tau_{j}^{2}+b \tau_{j}+c\right)\right] \\
& =a \xi_{j+1} \xi_{j+2}+b \frac{\xi_{j+1}+\xi_{j+2}}{2}+c .
\end{aligned}
$$




\subsection{B-Spline Representation of Splines}

In the previous section we have derived an explicit expression for the B-spline coefficients of polynomials; see (3.22). The next theorem extends this result by providing an explicit expression for the B-spline coefficients of any spline in $\mathbb{S}_{p, \boldsymbol{\xi}}$.

Theorem 23 (Representation of B-Spline Coefficients). Any element $s$ in the space $\mathbb{S}_{p, \xi}$ can be represented as ${ }^{4}$

$$
s(x)=\sum_{j=1}^{n} \Lambda_{j, p, \boldsymbol{\xi}}(s) B_{j, p, \boldsymbol{\xi}}(x), \quad x \in\left[\xi_{p+1}, \xi_{n+1}\right]
$$

where

$$
\Lambda_{j, p, \boldsymbol{\xi}}(s):=\frac{1}{p !} \begin{cases}\sum_{r=\mu_{j}}^{p}(-1)^{p-r} D^{r} \psi_{j, p, \boldsymbol{\xi}}\left(\tau_{j}\right) D_{+}^{p-r} s\left(\tau_{j}\right), & \text { if } \tau_{j}=\xi_{j}, \\ \sum_{r=\mu_{j}}^{p}(-1)^{p-r} D^{r} \psi_{j, p, \boldsymbol{\xi}}\left(\tau_{j}\right) D^{p-r} s\left(\tau_{j}\right), & \text { if } \xi_{j}<\tau_{j}<\xi_{j+p+1}, \\ \sum_{r=\mu_{j}}^{p}(-1)^{p-r} D^{r} \psi_{j, p, \boldsymbol{\xi}}\left(\tau_{j}\right) D_{-}^{p-r} s\left(\tau_{j}\right), & \text { if } \tau_{j}=\xi_{j+p+1},\end{cases}
$$

and where $\mu_{j} \geq 0$ is the number of times $\tau_{j}$ appears in $\xi_{j+1}, \ldots, \xi_{j+p}$.

Proof. Suppose $\xi_{j} \leq \tau_{j}<\xi_{j+p+1}$ and let $I_{j}:=\left[\xi_{m_{j}}, \xi_{m_{j}+1}\right)$ be the interval containing $\tau_{j}$. The restriction of $s$ to $I_{j}$ is a polynomial and so by Proposition 21 we find

$$
s(x)=\sum_{i=m_{j}-p}^{m_{j}}\left(\frac{1}{p !} \sum_{r=0}^{p}(-1)^{p-r} D^{r} \psi_{i, p, \boldsymbol{\xi}}\left(\tau_{j}\right) D_{+}^{p-r} s\left(\tau_{j}\right)\right) B_{i, p, \boldsymbol{\xi}}(x), \quad x \in I_{j} .
$$

Note that since $\xi_{j} \leq \tau_{j}<\xi_{j+p+1}$ we have $j \leq m_{j} \leq j+p$ which implies $m_{j}-p \leq$ $j \leq m_{j}$. By taking $i=j$ in (3.27) and using the local linear independence of the B-splines, we obtain

$$
\Lambda_{j, p, \boldsymbol{\xi}}(s):=\frac{1}{p !} \sum_{r=0}^{p}(-1)^{p-r} D^{r} \psi_{j, p, \boldsymbol{\xi}}\left(\tau_{j}\right) D_{+}^{p-r} s\left(\tau_{j}\right)
$$

Since $D^{r} \psi_{j, p, \boldsymbol{\xi}}\left(\tau_{j}\right)=0$ for $r<\mu_{j}$ we obtain the top term in (3.26). In the middle term we can replace $D_{+}^{p-r} s\left(\tau_{j}\right)$ by $D^{p-r} s\left(\tau_{j}\right)$ since $s \in C^{p-\mu_{j}}\left(\tau_{j}\right)$. The proof of the last term is similar using $D_{-}$instead of $D_{+}$.

Note that the operator $\Lambda_{j, p, \xi}$ in (3.24) is identical to $\Lambda_{j, p, \xi}$ in (3.26). However, in the spline case we need the restriction $\tau_{j} \in\left[\xi_{j}, \xi_{j+p+1}\right]$.

Because the set of B-splines $\left\{B_{j, p, \xi}\right\}_{j=1}^{n}$ is a basis for the space $\mathbb{S}_{p, \xi}$, the coefficients $\Lambda_{j, p, \xi}(s)$ are uniquely determined for any $s \in \mathbb{S}_{p, \xi}$. Thus, the right-hand side in (3.26) does not depend on the choice of $\tau_{j}$. This is an astonishing property

\footnotetext{
${ }^{4}$ The number $\Lambda_{j, p, \boldsymbol{\xi}}(s)$ is known as the de Boor-Fix functional [7] applied to $s$.
} 
considering the complexity of the expression. For example, one could take the Greville point $\xi_{j, p, \xi}^{*}$ defined in (3.21) as a valid choice for the point $\tau_{j}$. It is easy to verify that $\xi_{j, p, \boldsymbol{\xi}}^{*} \in\left[\xi_{j}, \xi_{j+p+1}\right]$, and moreover, $\xi_{j, p, \boldsymbol{\xi}}^{*} \in\left(\xi_{j}, \xi_{j+p+1}\right)$ if $B_{j, p, \boldsymbol{\xi}}$ is a continuous function.

Example 24. We consider the quadratic spline

$$
s(x)=\sum_{j=1}^{n} c_{j} B_{j, 2, \boldsymbol{\xi}}(x),
$$

and we illustrate that some derivative terms in the expression (3.26) can be canceled by specific choices of $\tau_{j}$. Assume for simplicity $\xi_{j}<\xi_{j+1}<\xi_{j+2}<\xi_{j+3}$.

- If $\tau_{j}$ is the Greville point $\xi_{j, 2, \xi}^{*}:=\left(\xi_{j+1}+\xi_{j+2}\right) / 2$, then there is no first derivative term. Indeed, we have

$$
c_{j}=\Lambda_{j, 2, \boldsymbol{\xi}}(s)=s\left(\xi_{j, 2, \boldsymbol{\xi}}^{*}\right)-\frac{\left(\xi_{j+2}-\xi_{j+1}\right)^{2}}{8} D^{2} s\left(\xi_{j, 2, \boldsymbol{\xi}}^{*}\right) .
$$

Moreover, since $s \in \mathbb{P}_{2}$ on $\left[\xi_{j+1}, \xi_{j+2}\right]$, we can replace $D^{2} s\left(\xi_{j, 2, \xi}^{*}\right)$ by a difference quotient

$$
D^{2} s\left(\xi_{j, 2, \boldsymbol{\xi}}^{*}\right)=\left(s\left(\xi_{j+2}\right)-2 s\left(\xi_{j, 2, \boldsymbol{\xi}}^{*}\right)+s\left(\xi_{j+1}\right)\right) /\left(\frac{\xi_{j+2}-\xi_{j+1}}{2}\right)^{2},
$$

to obtain

$$
c_{j}=-\frac{1}{2} s\left(\xi_{j+1}\right)+2 s\left(\xi_{j, 2, \boldsymbol{\xi}}^{*}\right)-\frac{1}{2} s\left(\xi_{j+2}\right) .
$$

- If $\tau_{j}$ is equal to $\xi_{j+1}$ or $\xi_{j+2}$, then there is no second derivative term. Indeed, we have

$$
c_{j}=\Lambda_{j, 2, \boldsymbol{\xi}}(s)=s\left(\tau_{j}\right)+\frac{\xi_{j, 2, \boldsymbol{\xi}}^{*}-\tau_{j}}{2} D s\left(\tau_{j}\right), \quad \tau_{j} \in\left\{\xi_{j+1}, \xi_{j+2}\right\}
$$

A similar property holds for any $p$ : if $\tau_{j}$ is chosen as one of the interior knots $\xi_{j+1}, \ldots, \xi_{j+p}$, then there is no $p$-th derivative term in the expression of $\Lambda_{j, p, \boldsymbol{\xi}}(s)$.

\subsection{Knot Insertion}

In this section we are addressing the problem of representing a given spline on a refined knot sequence. In particular, we focus on the special case where only a single knot is inserted. Since any refined knot sequence can be reached by repeatedly inserting one knot at a time, it suffices to deal with this case.

Without loss of generality, we assume that the spline $s=\sum_{j=1}^{n} c_{j} B_{j, p, \xi}$ is given on a $(p+1)$-basic knot sequence $\xi:=\left\{\xi_{i}\right\}_{i=1}^{n+p+1}$. We want to insert a knot $\xi$ in some subinterval $\left[\xi_{m}, \xi_{m+1}\right)$ of $\left[\xi_{p+1}, \xi_{n+1}\right)$, resulting in a new $(p+1)$-basic knot sequence $\tilde{\xi}:=\left\{\tilde{\xi}_{i}\right\}_{i=1}^{n+p+2}$ defined by

$$
\tilde{\xi}_{i}:= \begin{cases}\xi_{i}, & \text { if } 1 \leq i \leq m \\ \xi, & \text { if } i=m+1 \\ \xi_{i-1}, & \text { if } m+2 \leq i \leq n+p+2\end{cases}
$$


The B-spline form of $s$ on the new knot sequence can be computed with the aid of the following procedure introduced by Böhm [3].

Theorem 25 (Knot Insertion). Let the $(p+1)$-basic knot sequence $\tilde{\xi}:=\left\{\tilde{\xi}_{i}\right\}_{i=1}^{n+p+2}$ be obtained from the $(p+1)$-basic knot sequence $\xi:=\left\{\xi_{i}\right\}_{i=1}^{n+p+1}$ by inserting just one knot $\xi$, such that $\xi_{m} \leq \xi<\xi_{m+1}$ as in (3.29). Then,

$$
s(x)=\sum_{j=1}^{n} c_{j} B_{j, p, \boldsymbol{\xi}}(x)=\sum_{i=1}^{n+1} \tilde{c}_{i} B_{i, p, \tilde{\boldsymbol{\xi}}}(x), \quad x \in\left[\xi_{p+1}, \xi_{n+1}\right],
$$

where

$$
\tilde{c}_{i}= \begin{cases}c_{i}, & \text { if } i \leq m-p, \\ \frac{\xi-\xi_{i}}{\xi_{i+p}-\xi_{i}} c_{i}+\frac{\xi_{i+p}-\xi}{\xi_{i+p}-\xi_{i}} c_{i-1}, & \text { if } m-p<i \leq m, \\ c_{i-1}, & \text { if } i>m .\end{cases}
$$

Proof. From Theorem 15 it follows that $\mathbb{S}_{p, \boldsymbol{\xi}}$ is a subspace of $\mathbb{S}_{p, \tilde{\xi}}$, since we have reduced the continuity requirement at $\xi_{m}$ if $\xi=\xi_{m}$ or introduced another segment otherwise. Hence, the B-splines in $\mathbb{S}_{p, \boldsymbol{\xi}}$ belong to $\mathbb{S}_{p, \tilde{\boldsymbol{\xi}}}$, and we can write

$$
B_{j, p, \xi}=\sum_{i=1}^{n+1} \alpha_{i, j, p} B_{i, p, \tilde{\boldsymbol{\xi}}}, \quad j=1, \ldots, n
$$

for some real numbers $\alpha_{i, j, p}$. Suppose $s \in \mathbb{S}_{p, \boldsymbol{\xi}}$ is given by (3.30). Then,

$$
\sum_{j=1}^{n} c_{j} B_{j, p, \boldsymbol{\xi}}=\sum_{i=1}^{n+1}\left(\sum_{j=1}^{n} \alpha_{i, j, p} c_{j}\right) B_{i, p, \tilde{\boldsymbol{\xi}}}
$$

By linear independence of the B-splines in $\mathbb{S}_{p, \tilde{\xi}}$ we obtain

$$
\tilde{c}_{i}=\sum_{j=1}^{n} \alpha_{i, j, p} c_{j}, \quad i=1, \ldots, n+1
$$

Note that each $\alpha_{i, j, p}$ is independent of the $c$ 's.

Now, consider the function $f_{y}(x)=(y-x)^{p}$ for fixed $y \in \mathbb{R}$. By the Marsden identity (3.15) we have

$$
(y-x)^{p}=\sum_{j=1}^{n} c_{j} B_{j, p, \boldsymbol{\xi}}(x)=\sum_{i=1}^{n+1} \tilde{c}_{i} B_{i, p, \tilde{\boldsymbol{\xi}}}(x), \quad x \in\left[\xi_{p+1}, \xi_{n+1}\right], \quad y \in \mathbb{R},
$$

where

$$
c_{j}=\psi_{j, p, \boldsymbol{\xi}}(y)=\left(y-\xi_{j+1}\right) \cdots\left(y-\xi_{j+p}\right)
$$

and

$$
\tilde{c}_{i}=\psi_{i, p, \tilde{\boldsymbol{\xi}}}(y)=\left(y-\tilde{\xi}_{i+1}\right) \cdots\left(y-\tilde{\xi}_{i+p}\right) .
$$


Hence, for the function $f_{y}(x)$, the identity (3.32) takes the form

$$
\psi_{i, p, \tilde{\boldsymbol{\xi}}}(y)=\sum_{j=1}^{n} \alpha_{i, j, p} \psi_{j, p, \boldsymbol{\xi}}(y), \quad i=1, \ldots, n+1
$$

From the relation (3.29) between the knot sequences $\tilde{\xi}$ and $\boldsymbol{\xi}$, we deduce that $\psi_{i, p, \tilde{\boldsymbol{\xi}}}=\psi_{i, p, \boldsymbol{\xi}}$ for $i \leq m-p$, and $\psi_{i, p, \tilde{\boldsymbol{\xi}}}=\psi_{i-1, p, \boldsymbol{\xi}}$ for $i>m$, and using the dual recurrence relation (2.11) that for $m-p<i \leq m$,

$$
\psi_{i, p, \tilde{\boldsymbol{\xi}}}(y)=(y-\xi) \psi_{i, p-1, \boldsymbol{\xi}}(y)=\frac{\xi-\xi_{i}}{\xi_{i+p}-\xi_{i}} \psi_{i, p, \boldsymbol{\xi}}+\frac{\xi_{i+p}-\xi}{\xi_{i+p}-\xi_{i}} \psi_{i-1, p, \boldsymbol{\xi}} .
$$

Then, (3.31) follows from (3.32) and (3.33).

When several knots have to be inserted simultaneously, alternative algorithms can be used instead of repeating the single knot insertion procedure given in Theorem 25. In Section 4.3 we provide such a simultaneous knot insertion algorithm in case of uniform knot sequences. A more general (but also more complex) knot insertion algorithm is known as the Oslo algorithm [11].

- Convex Combination. From relation (3.31) we see that the coefficients $\tilde{c}_{i}$ are a convex combination of the coefficients $c_{i}$. In general, the coefficients obtained after repeated knot insertion are a convex combination of the original coefficients.

- Evaluation. Repeated knot insertion gives rise to an evaluation process for spline functions in B-spline form. Indeed, the evaluation of a spline $s$ at the point $x$ can be achieved by the repeated insertion of $x$ as a knot till it has multiplicity $p$. Then, assuming that for some $m$,

$$
\xi_{m}<x=\xi_{m+1}=\cdots=\xi_{m+p}<\xi_{m+p+1},
$$

we can conclude from (2.29) and (3.19) that

$$
B_{j, p, \xi}(x)= \begin{cases}1, & \text { if } j=m, \\ 0, & \text { otherwise }\end{cases}
$$

and

$$
s(x)=\sum_{j=1}^{n} c_{j} B_{j, p, \boldsymbol{\xi}}(x)=c_{m} B_{m, p, \boldsymbol{\xi}}(x)=c_{m} .
$$

When comparing (3.31) with (3.9), we observe that single knot insertion is nothing else than applying once the B-spline coefficient recurrence relation. This evaluation procedure is a fast and numerically stable algorithm introduced by de Boor [4]. 


\subsection{Condition Number}

A basis $\left\{B_{j}\right\}$ of a normed space is said to be stable with respect to a vector norm if there are positive constants $K_{L}$ and $K_{U}$ such that

$$
K_{L}^{-1}\|\boldsymbol{c}\| \leq\left\|\sum_{j} c_{j} B_{j}\right\| \leq K_{U}\|\boldsymbol{c}\|,
$$

for all coefficient vectors $c:=\left(c_{j}\right)$. For simplicity we use the same symbol $\|\cdot\|$ for the norm in the space and the vector norm. The number

$$
\kappa:=\inf \left\{K_{L} K_{U}: K_{L} \text { and } K_{U} \text { satisfy (3.34) }\right\}
$$

is called the condition number of the basis $\left\{B_{j}\right\}$ with respect to $\|\cdot\|$.

Such condition numbers give an upper bound for how much an error in coefficients can be magnified in function values and vice versa. Indeed, if $f=\sum_{j} c_{j} B_{j} \neq 0$ and $g=\sum_{j} d_{j} B_{j}$ then it follows immediately from (3.34) that

$$
\frac{1}{\kappa} \frac{\|\boldsymbol{c}-\boldsymbol{d}\|}{\|\boldsymbol{c}\|} \leq \frac{\|f-g\|}{\|f\|} \leq \kappa \frac{\|\boldsymbol{c}-\boldsymbol{d}\|}{\|\boldsymbol{c}\|}
$$

where $\boldsymbol{c}:=\left(c_{j}\right)$ and $\boldsymbol{d}:=\left(d_{j}\right)$. Many other applications are given in [5] and it is interesting to have estimates for the size of $\kappa$.

We consider the $L_{q}$-norm for functions and the $q$-norm for vectors with $1 \leq q \leq$ $\infty$. We focus on a scaled version of the B-spline basis defined on $\left[\xi_{1}, \xi_{n+p+1}\right]$,

$$
\left\{N_{j, p, q, \boldsymbol{\xi}}\right\}_{j=1}^{n}:=\left\{\gamma_{j, p, \boldsymbol{\xi}}^{-1 / q} B_{j, p, \boldsymbol{\xi}}\right\}_{j=1}^{n}
$$

where $\gamma_{j, p, \boldsymbol{\xi}}:=\left(\xi_{j+p+1}-\xi_{j}\right) /(p+1)$; see also (2.30). The knot sequence $\boldsymbol{\xi}$ is assumed to be $(p+1)$-basic in order to have linearly independent B-splines. This also ensures that $\gamma_{j, p, \boldsymbol{\xi}}>0$. The $q$-norm condition number of the basis in (3.36) will be denoted by $\kappa_{p, q, \xi}$, i.e.,

$$
\kappa_{p, q, \boldsymbol{\xi}}:=\sup _{\boldsymbol{c} \neq 0} \frac{\left\|\sum_{j=1}^{n} c_{j} N_{j, p, q, \boldsymbol{\xi}}\right\|_{L_{q}\left(\left[\xi_{1}, \xi_{n+p+1}\right]\right)}}{\|\boldsymbol{c}\|_{q}} \sup _{\boldsymbol{c} \neq 0} \frac{\|\boldsymbol{c}\|_{q}}{\left\|\sum_{j=1}^{n} c_{j} N_{j, p, q, \boldsymbol{\xi}}\right\|_{L_{q}\left(\left[\xi_{1}, \xi_{n+p+1}\right]\right)}} .
$$

The next theorem shows that the scaled B-spline basis above is stable in any $L_{q^{-}}$ norm independently on the knot sequence $\boldsymbol{\xi}$. It also provides an upper bound for the $q$-norm condition number which does not depend on $\boldsymbol{\xi}$. To this end, we first state the Hölder inequality for sums:

$$
\sum_{j=1}^{n}\left|x_{j} y_{j}\right| \leq\|\boldsymbol{x}\|_{q}\|\boldsymbol{y}\|_{q^{\prime}}
$$

where $q, q^{\prime}$ are integers so that 


$$
\frac{1}{q}+\frac{1}{q^{\prime}}=1, \quad 1 \leq q \leq \infty
$$

In particular, $q^{\prime}=\infty$ if $q=1$ and $q^{\prime}=2$ if $q=2$.

Theorem 26. For any $p \geq 0$ there exists a positive constant $K_{p}$ depending only on $p$, such that for any vector $c:=\left(c_{1}, \ldots, c_{n}\right)$ and for any $1 \leq q \leq \infty$ we have

$$
K_{p}^{-1}\|\boldsymbol{c}\|_{q} \leq\left\|\sum_{j=1}^{n} c_{j} N_{j, p, q, \xi}\right\|_{L_{q}\left(\left[\xi_{1}, \xi_{n+p+1}\right]\right)} \leq\|\boldsymbol{c}\|_{q}
$$

Proof. We first prove the upper inequality. By using the nonnegative partition of unity property of B-splines, the upper bound for $q=\infty$ is straightforward. For $q=1$, we have

$$
\int_{\xi_{1}}^{\xi_{n+p+1}}\left|\sum_{j=1}^{n} c_{j} N_{j, p, q, \boldsymbol{\xi}}(x)\right| \mathrm{d} x \leq \sum_{j=1}^{n}\left|c_{j}\right| \gamma_{j, p, \boldsymbol{\xi}}^{-1} \int_{\xi_{j}}^{\xi_{j+p+1}} B_{j, p, \boldsymbol{\xi}}(x) \mathrm{d} x=\|\boldsymbol{c}\|_{1} .
$$

Finally, we consider $1<q<\infty$. By applying the Hölder inequality (3.38) and again the nonnegative partition of unity property of B-splines, we obtain for $x \in$ $\left[\xi_{1}, \xi_{n+p+1}\right]$,

$$
\begin{aligned}
\left|\sum_{j=1}^{n} c_{j} N_{j, p, q, \boldsymbol{\xi}}(x)\right| & \leq \sum_{j=1}^{n}\left|c_{j} \gamma_{j, p, \boldsymbol{\xi}}^{-1 / q} B_{j, p, \boldsymbol{\xi}}(x)(x)^{1 / q}\right|\left|B_{j, p, \boldsymbol{\xi}}(x)\right|^{1-1 / q} \\
& \leq\left(\sum_{j=1}^{n}\left|c_{j}\right|^{q} \gamma_{j, p, \boldsymbol{\xi}}^{-1} B_{j, p, \boldsymbol{\xi}}(x)\right)^{1 / q}\left(\sum_{j=1}^{n} B_{j, p, \boldsymbol{\xi}}(x)\right)^{1-1 / q} \\
& \leq\left(\sum_{j=1}^{n}\left|c_{j}\right|^{q} \gamma_{j, p, \boldsymbol{\xi}}^{-1} B_{j, p, \boldsymbol{\xi}}(x)\right)^{1 / q} .
\end{aligned}
$$

Raising both sides of this inequality to the $q$-th power and integrating gives the inequality

$$
\int_{\xi_{1}}^{\xi_{n+p+1}}\left|\sum_{j=1}^{n} c_{j} N_{j, p, q, \boldsymbol{\xi}}(x)\right|^{q} \mathrm{~d} x \leq \sum_{j=1}^{n}\left|c_{j}\right|^{q} \gamma_{j, p, \boldsymbol{\xi}}^{-1} \int_{\xi_{j}}^{\xi_{j+p+1}} B_{j, p, \boldsymbol{\xi}}(x) \mathrm{d} x=\|\boldsymbol{c}\|_{q}^{q} .
$$

Taking the $q$-th roots on both sides proves the upper inequality in (3.40).

We now focus on the lower inequality. We extend $\boldsymbol{\xi}$ to a $(p+1)$-open knot sequence $\hat{\xi}$ by possibly increasing the multiplicity of $\xi_{1}$ and $\xi_{n+p+1}$ to $p+1$. Clearly, the set of B-splines on $\xi$ is a subset of the set of B-splines on $\hat{\xi}$, and any linear combination of the B-splines on $\boldsymbol{\xi}$ is a linear combination of the B-splines on $\hat{\xi}$ where the extra B-splines have coefficients zero. Therefore, without loss of generality, we can assume that the knot sequence is open with the basic interval $\left[\xi_{1}, \xi_{n+p+1}\right]$. The lower bound then follows from Lemma 46; see Section 5.3.1.

Finally, we define a condition number that is independent of the knot sequence, 


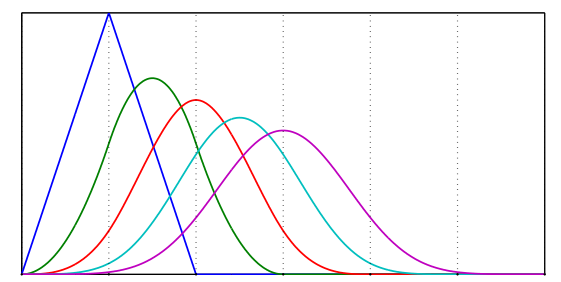

Fig. 3 The cardinal B-splines $M_{p}$ for $p=1, \ldots, 5$. The uniform knot positions are visualized by vertical dotted lines.

$$
\kappa_{p, q}:=\sup _{\xi} \kappa_{p, q, \boldsymbol{\xi}}
$$

Theorem 26 shows that

$$
\kappa_{p, q} \leq K_{p}<\infty .
$$

It is known that $\kappa_{p, q}$ grows like $2^{p}$ for all $1 \leq q \leq \infty$; see $[34,40]$ where it is proved that

$$
\frac{1}{p+1} 2^{p-1 / 2} \leq \kappa_{p, q} \leq(p+1) 2^{p+1}, \quad 1 \leq q \leq \infty .
$$

\section{Cardinal B-Splines}

A particularly interesting case of B-spline functions is obtained when the knot sequence is uniformly spaced. Without loss of generality, we can assume that the knot sequence is given by the set of integers $\mathbb{Z}$. It is natural to index the knots as $\xi_{j}=j$, $j \in \mathbb{Z}$. Due to the translation invariance property (2.9) we have

$$
B_{j, p, \mathbb{Z}}(x)=B_{0, p, \mathbb{Z}}(x-j), \quad j \in \mathbb{Z}, \quad x \in \mathbb{R} .
$$

Therefore, all the B-splines on the knot sequence $\mathbb{Z}$ are integer translates of a single function. This motivates the following definition.

Definition 27. The function $M_{p}:=B[0,1, \ldots, p+1]$ is the cardinal B-spline of degree $p$.

Example 28. Figure 3 illustrates the cardinal B-splines $M_{p}$ for $p=1, \ldots, 5$.

\subsection{Main Properties}

Cardinal B-splines possess several interesting features. Of course, they inherit all the properties of general B-splines, and in particular the following ones. 
- Local Support. From (2.6) it follows that the cardinal B-spline $M_{p}$ is locally supported on the interval $[0, p+1]$.

- Nonnegativity, Piecewise Structure and Smoothness. From (2.7), (2.8) and (2.26) it follows that the cardinal B-spline $M_{p}$ is a nonnegative, piecewise polynomial of degree $p$ belonging to the class $C^{p-1}(\mathbb{R})$.

- Differentiation and Integration. The formulas (2.22) and (2.30) simplify in the case of cardinal B-splines to

$$
D_{+} M_{p}(x)=M_{p-1}(x)-M_{p-1}(x-1), \quad p \geq 1,
$$

and

$$
\int_{\mathbb{R}} M_{p}(x) \mathrm{d} x=1 .
$$

- Recurrence Relation. From Definition 2 we obtain the following recurrence relation for cardinal B-splines,

$$
\begin{aligned}
& M_{0}(x)= \begin{cases}1, & \text { if } x \in[0,1), \\
0, & \text { otherwise, }\end{cases} \\
& M_{p}(x)=\frac{x}{p} M_{p-1}(x)+\frac{p+1-x}{p} M_{p-1}(x-1), \quad p \geq 1 .
\end{aligned}
$$

The uniformity of the knot sequence endows the cardinal B-splines with several additional properties. A key feature is based on convolution.

- Convolution. The convolution of two functions $f$ and $g$ is defined by

$$
(f * g)(x):=\int_{\mathbb{R}} f(x-y) g(y) \mathrm{d} y .
$$

The cardinal B-spline $M_{p}$ can be characterized using convolution by

$$
M_{p}(x)=\left(M_{p-1} * M_{0}\right)(x)=\int_{0}^{1} M_{p-1}(x-y) \mathrm{d} y, \quad p \geq 1,
$$

and

$$
M_{p}(x)=(\overbrace{M_{0} * \cdots * M_{0}}^{p+1})(x) .
$$

Proof. From (4.2) we deduce

$$
\begin{aligned}
M_{p}(x) & =\int_{0}^{x}\left(M_{p-1}(y)-M_{p-1}(y-1)\right) \mathrm{d} y=\int_{0}^{x} M_{p-1}(y) \mathrm{d} y-\int_{-1}^{x-1} M_{p-1}(y) \mathrm{d} y \\
& =\int_{x-1}^{x} M_{p-1}(y) \mathrm{d} y=\int_{0}^{1} M_{p-1}(x-y) \mathrm{d} y .
\end{aligned}
$$

Applying recursively (4.6) immediately gives (4.7). 
- Fourier Transform. The Fourier transform of a function $f \in L_{2}(\mathbb{R})$ is defined by

$$
\widehat{f}(\theta):=\int_{\mathbb{R}} f(x) \mathrm{e}^{-\mathrm{i} \theta x} \mathrm{~d} x,
$$

where $\mathrm{i}:=\sqrt{-1}$ denotes the imaginary unit. The Fourier transform of the cardinal B-spline $M_{p}$ is given by

$$
\widehat{M_{p}}(\theta)=\left(\frac{1-\mathrm{e}^{-\mathrm{i} \theta}}{\mathrm{i} \theta}\right)^{p+1} .
$$

Proof. From (4.4), a direct computation gives

$$
\widehat{M_{0}}(\theta)=\frac{1-\mathrm{e}^{-\mathrm{i} \theta}}{\mathrm{i} \theta}
$$

An interesting property of the Fourier transform of a convolution is

$$
(\widehat{f * g})(\theta)=\widehat{f}(\theta) \widehat{g}(\theta), \quad \forall f, g \in L_{2}(\mathbb{R}) ;
$$

see, e.g., [39]. Hence, by combining (4.9) with (4.7) we deduce that $\widehat{M_{p}}(\theta)=$ $\left(\widehat{M_{0}}(\theta)\right)^{p+1}$, which implies (4.8).

- Symmetry. The cardinal B-spline $M_{p}$ is symmetric with respect to the midpoint of its support, namely $(p+1) / 2$. More generally,

$$
D^{r} M_{p}\left(\frac{p+1}{2}+x\right)=(-1)^{r} D^{r} M_{p}\left(\frac{p+1}{2}-x\right), \quad r=0, \ldots, p-1,
$$

and

$$
D_{-}^{p} M_{p}\left(\frac{p+1}{2}+x\right)=(-1)^{p} D_{+}^{p} M_{p}\left(\frac{p+1}{2}-x\right) .
$$

Proof. It suffices to prove that $M_{p}(p+1-x)=M_{p}(x)$. The general result then follows from repeated differentiations. We proceed by induction. It is easy to check that it is true for $p=0$. Assuming the symmetry property holds for degree $p-1$ and using (4.6), we get

$$
\begin{aligned}
M_{p}(p+1-x) & =\int_{0}^{1} M_{p-1}(p+1-x-t) \mathrm{d} t=\int_{0}^{1} M_{p-1}(x-1+t) \mathrm{d} t \\
& =-\int_{0}^{1} M_{p-1}(x-t) \mathrm{d} t=\int_{0}^{1} M_{p-1}(x-t) \mathrm{d} t=M_{p}(x) .
\end{aligned}
$$

We now focus on the set of integer translates of the cardinal B-spline $M_{p}$, i.e.,

$$
\left\{M_{p}(\cdot-j), j \in \mathbb{Z}\right\} .
$$

They have the following properties. 
- Linear Independence. From (4.1) it follows that the integer translates $M_{p}(\cdot-j)$, $j \in \mathbb{Z}$, are (locally) linearly independent on $\mathbb{R}$. They span the space of piecewise polynomials of degree $p$ and smoothness $p-1$ with integer break points; see (3.12).

- Partition of Unity. From (3.19) and (4.1) we get

$$
\sum_{j \in \mathbb{Z}} M_{p}(x-j)=1, \quad x \in \mathbb{R} .
$$

Due to the local support of cardinal B-splines, the above series reduces to a finite sum for any $x$. More precisely, referring to (2.21), we have

$$
\sum_{j=m-p}^{m} M_{p}(x-j)=1, \quad x \in[m, m+1) .
$$

- Greville Points. From (3.21)-(3.20) and (4.1) we have

$$
x=\sum_{j \in \mathbb{Z}} \zeta_{j, p}^{*} M_{p}(x-j), \quad x \in \mathbb{R},
$$

with

$$
\zeta_{j, p}^{*}:=\frac{(1+j)+\cdots+(p+j)}{p}=\frac{p+1}{2}+j .
$$

\subsection{Inner Products}

Inner products of cardinal B-splines and their translates can be interpreted as evaluations of higher-degree cardinal B-splines; similar results also hold for their derivatives ${ }^{5}$.

Theorem 29 (Inner Product). Given $p_{1}, p_{2} \geq 0$, we have

$$
\int_{\mathbb{R}} M_{p_{1}}(y) M_{p_{2}}(y+x) \mathrm{d} y=M_{p_{1}+p_{2}+1}\left(p_{1}+1+x\right)=M_{p_{1}+p_{2}+1}\left(p_{2}+1-x\right) .
$$

Proof. From the symmetry property (4.10)-(4.11) and the convolution relation (4.6) of cardinal B-splines, we get

5 The inner product formula for cardinal B-splines traces back to [44]. The formula for derivatives of cardinal B-splines can be found in [21] and a generalization for multivariate box splines in [48]. 


$$
\begin{aligned}
\int_{\mathbb{R}} M_{p_{1}}(y) M_{p_{2}}(y+x) \mathrm{d} y & =\int_{\mathbb{R}} M_{p_{1}}(y) M_{p_{2}}\left(p_{2}+1-y-x\right) \mathrm{d} y \\
& =\left(M_{p_{1}} * M_{p_{2}}\right)\left(p_{2}+1-x\right) \\
& =(\overbrace{M_{0} * \cdots * M_{0}}^{p_{1}+1} * \overbrace{M_{0} * \cdots * M_{0}}^{p_{2}+1})\left(p_{2}+1-x\right) \\
& =M_{p_{1}+p_{2}+1}\left(p_{2}+1-x\right) .
\end{aligned}
$$

Finally, again by symmetry of cardinal B-splines, we have

$$
M_{p_{1}+p_{2}+1}\left(p_{1}+1+x\right)=M_{p_{1}+p_{2}+1}\left(p_{2}+1-x\right),
$$

which completes the proof.

Theorem 30 (Inner Product of Derivatives). Given $p_{1} \geq r_{1} \geq 0$ and $p_{2} \geq r_{2} \geq 0$, we have

$$
\begin{aligned}
\int_{\mathbb{R}} D_{+}^{r_{1}} M_{p_{1}}(y) D_{+}^{r_{2}} M_{p_{2}}(y+x) \mathrm{d} y & =(-1)^{r_{1}} D^{r_{1}+r_{2}} M_{p_{1}+p_{2}+1}\left(p_{1}+1+x\right) \\
& =(-1)^{r_{2}} D^{r_{1}+r_{2}} M_{p_{1}+p_{2}+1}\left(p_{2}+1-x\right) .
\end{aligned}
$$

Proof. Because of the (anti-)symmetry of higher order derivatives of cardinal Bsplines given in (4.10), we have

$$
\begin{aligned}
& (-1)^{r_{1}} D^{r_{1}+r_{2}} M_{p_{1}+p_{2}+1}\left(p_{1}+1+x\right) \\
& \quad=(-1)^{r_{1}}(-1)^{r_{1}+r_{2}} D^{r_{1}+r_{2}} M_{p_{1}+p_{2}+1}\left(p_{1}+p_{2}+2-\left(p_{1}+1+x\right)\right) \\
& \quad=(-1)^{r_{2}} D^{r_{1}+r_{2}} M_{p_{1}+p_{2}+1}\left(p_{2}+1-x\right) .
\end{aligned}
$$

So, we only have to show one of both equalities in the theorem. This can be proved by induction on the order of derivatives. The base case $\left(r_{1}=r_{2}=0\right)$ simply follows from Theorem 29. We consider two inductive steps: in the first inductive step we increase the order of derivative of $M_{p_{1}}$ by one, i.e., $r_{1} \rightarrow r_{1}+1$, and in the second inductive step we increase the order of derivative of $M_{p_{2}}$ by one, i.e., $r_{2} \rightarrow r_{2}+1$.

1. $\left(r_{1} \rightarrow r_{1}+1\right)$. Using (4.2) and the induction hypothesis, we have

$$
\begin{aligned}
\int_{\mathbb{R}} & D_{+}^{r_{1}+1} M_{p_{1}}(y) D_{+}^{r_{2}} M_{p_{2}}(y+x) \mathrm{d} y \\
& =\int_{\mathbb{R}}\left(D_{+}^{r_{1}} M_{p_{1}-1}(y)-D_{+}^{r_{1}} M_{p_{1}-1}(y-1)\right) D_{+}^{r_{2}} M_{p_{2}}(y+x) \mathrm{d} y \\
& =\int_{\mathbb{R}} D_{+}^{r_{1}} M_{p_{1}-1}(y) D_{+}^{r_{2}} M_{p_{2}}(y+x) \mathrm{d} y-\int_{\mathbb{R}} D_{+}^{r_{1}} M_{p_{1}-1}(y-1) D_{+}^{r_{2}} M_{p_{2}}(y+x) \mathrm{d} y \\
& =(-1)^{r_{1}}\left(D^{r_{1}+r_{2}} M_{p_{1}+p_{2}}\left(p_{1}+x\right)-D^{r_{1}+r_{2}} M_{p_{1}+p_{2}}\left(p_{1}+1+x\right)\right) \\
& =(-1)^{r_{1}+1} D^{r_{1}+r_{2}+1} M_{p_{1}+p_{2}+1}\left(p_{1}+1+x\right) .
\end{aligned}
$$

2. $\left(r_{2} \rightarrow r_{2}+1\right)$. This inductive step can be proved in a completely analogous way as the first inductive step. 
Due to the relevance of the set (4.12), the results in Theorem 29 and Theorem 30 are of particular interest when we consider integer shifts, i.e., $x \in \mathbb{Z}$. In this case, the above inner products reduce to evaluations of cardinal B-splines and their derivatives at either integer or half-integer points. Moreover, there is a relation with the Greville points (4.13). Indeed, if $p_{1}=p_{2}=p$ and $x=i$ in Theorem 29, then

$$
\int_{\mathbb{R}} M_{p}(x) M_{p}(x+i) \mathrm{d} x=M_{2 p+1}(p+1+i)=M_{2 p+1}\left(\zeta_{i, 2 p+1}^{*}\right) .
$$

A similar relation holds for the inner products of derivatives in Theorem 30. Thanks to the recurrence relation for derivatives (4.2), the inner products of derivatives of cardinal B-splines and its integer translates reduce to evaluations of cardinal Bsplines at either integer or half-integer points.

\subsection{Uniform Knot Insertion}

In Section 3.5 we have seen how to insert a (single) knot into an existing knot sequence without changing the shape of a given spline function defined on that knot sequence. For uniform knot sequences, we can provide a simple alternative algorithm for inserting simultaneously a knot in each knot interval.

Let us consider the B-splines of degree $p$ over the uniform knot sequence given by $\mathbb{Z} / 2$. In this case, it is natural to index the knots as

$$
\xi_{i}=\left\{\begin{array}{ll}
k, & \text { if } i=2 k, \\
k+1 / 2, & \text { if } i=2 k+1,
\end{array} \quad i \in \mathbb{Z} .\right.
$$

From the definition we have $B_{i, p, \mathbb{Z} / 2}(x)=M_{p}(2 x-i)$ for $i \in \mathbb{Z}$. Since $\mathbb{S}_{p, \mathbb{Z}} \subset \mathbb{S}_{p, \mathbb{Z} / 2}$, the cardinal B-spline $M_{p}$ is a refinable function, i.e., it can be written as a linear combination of translated and dilated versions of itself:

$$
M_{p}(x)=\sum_{i=0}^{p+1} \alpha_{i, p} M_{p}(2 x-i) .
$$

We are now looking for a relation between the coefficients of a given spline function corresponding to knots in $\mathbb{Z}$ and the coefficients of the same function corresponding to knots in $\mathbb{Z} / 2$. The following simultaneous knot insertion procedure was introduced by Lane and Riesenfeld [32].

Theorem 31 (Uniform Knot Insertion). Consider the uniform knot sequences $\mathbb{Z}$ and $\mathbb{Z} / 2$. Then,

$$
s(x)=\sum_{j \in \mathbb{Z}} c_{j} M_{p}(x-j)=\sum_{i \in \mathbb{Z}} \tilde{c}_{i} M_{p}(2 x-i),
$$


with $\tilde{c}_{i}=\tilde{c}_{i}^{[p]}$ defined recursively by

$$
\tilde{c}_{i}^{[p]}:=\frac{\tilde{c}_{i}^{[p-1]}+\tilde{c}_{i-1}^{[p-1]}}{2},
$$

starting from

$$
\tilde{c}_{i}^{[0]}:= \begin{cases}c_{j}, & \text { if } i=2 j, \\ c_{j}, & \text { if } i=2 j+1 .\end{cases}
$$

Proof. For $p=0$ we can directly check that

$$
M_{0}(x)=M_{0}(2 x)+M_{0}(2 x-1),
$$

leading to (4.15) with (4.17). We proceed by induction on $p$. Assume the relation (4.15) with (4.16) holds for cardinal B-splines of degree $p-1$. Then, by using the convolution property (4.6) we get

$$
\begin{aligned}
\sum_{j \in \mathbb{Z}} c_{j} M_{p}(x-j)=\int_{0}^{1} \sum_{j \in \mathbb{Z}} c_{j} M_{p-1}(x-y-j) \mathrm{d} y=\int_{0}^{1} \sum_{i \in \mathbb{Z}} \tilde{c}_{i}^{[p-1]} M_{p-1}(2 x-2 y-i) \mathrm{d} y \\
=\sum_{i \in \mathbb{Z}} \tilde{c}_{i}^{[p-1]}\left(\int_{0}^{1 / 2} M_{p-1}(2 x-2 y-i) \mathrm{d} y+\int_{1 / 2}^{1} M_{p-1}(2 x-2 y-i) \mathrm{d} y\right) \\
=\sum_{i \in \mathbb{Z}} \frac{\tilde{c}_{i}^{[p-1]}}{2}\left(M_{p}(2 x-i)+M_{p}(2 x-i-1)\right)=\sum_{i \in \mathbb{Z}} \frac{\tilde{c}_{i}^{[p-1]}+\tilde{c}_{i-1}^{[p-1]}}{2} M_{p}(2 x-i),
\end{aligned}
$$

which concludes the proof.

The knot insertion procedure in Theorem 31 can be geometrically described as follows. First, every coefficient is doubled. Second, a sequence of $p$ sets of coefficients is constructed by taking averages of the previous set of coefficients.

The coefficients $\left\{\alpha_{i, p}\right\}$ in (4.14) can be directly computed from Theorem 31, and we obtain the explicit expression

$$
\alpha_{i, p}=\frac{1}{2^{p}}\left(\begin{array}{c}
p+1 \\
i
\end{array}\right), \quad i=0, \ldots, p+1 .
$$

They are called the subdivision mask of the (uniform) B-spline refinement scheme of degree $p$.

\section{Spline Approximation}

In this section we discuss how well a sufficiently smooth function can be approximated in the spline space spanned by a given set of B-splines. Exploiting the properties of the B-spline basis presented in the previous sections, we explicitly construct 
a spline which achieves optimal approximation accuracy for the function and its derivatives, and we determine the corresponding error estimates. The construction method we are going to present is local and linear.

\subsection{Preliminaries}

Let $I$ be a finite interval of the real line. A function $f: I \rightarrow \mathbb{R}$ is a piecewise continuous function on $I$ if it is bounded and continuous except at a finite number of points, where the value is obtained by taking the limit either from the left or the right. We denote the space of these functions by $C^{-1}(I)$.

For $r \in \mathbb{N}_{0}$ and $1 \leq q \leq \infty$ the one-dimensional Sobolev spaces are defined by

$$
W_{q}^{r}(I):=\left\{f: I \rightarrow \mathbb{R}: D^{j} f \in L_{q}(I), j=0, \ldots, r\right\} .
$$

They are normed spaces with norm

$$
\|f\|_{W_{q}^{r}(I)}^{2}:=\sum_{j=0}^{r}\left\|D^{j} f\right\|_{L_{q}(I)}^{2},
$$

called Sobolev norm. It can be shown that for $r \in \mathbb{N}$ and $1<q<\infty$,

$$
C^{r}(I) \subset W_{\infty}^{r}(I) \subset W_{q}^{r}(I) \subset W_{1}^{r}(I) \subset C^{r-1}(I) .
$$

The Hölder inequality for integrals is given by

$$
\int_{a}^{b}|f(x) g(x)| \mathrm{d} x \leq\|f\|_{L_{q}(I)}\|g\|_{L_{q^{\prime}}(I)}
$$

where $q, q^{\prime}$ are integers satisfying (3.39).

The Taylor polynomial of degree $p$ at the point $a$ to a function $f \in W_{1}^{p+1}([a, b])$ is defined by

$$
\mathscr{T}_{a, p} f(x):=\sum_{j=0}^{p} \frac{(x-a)^{j}}{j !} D^{j} f(a)
$$

and its approximation error can be expressed in integral form for $x \in[a, b]$ as

$$
f(x)-\mathscr{T}_{a, p} f(x)=\frac{1}{p !} \int_{a}^{b}(x-y)_{+}^{p} D^{p+1} f(y) \mathrm{d} y .
$$

Every polynomial $g \in \mathbb{P}_{p}$ can be written in Taylor form as $g=\mathscr{T}_{a, p} g$.

Theorem 32 (Taylor Interpolation Error). Let $f \in W_{q}^{p+1}([a, b])$ with $1 \leq q \leq \infty$, and let $\mathscr{T}_{a, p} f$ be the Taylor polynomial of degree $p$ to $f$ at the point $a$. Then, for any $x \in[a, b]$ and $0 \leq r \leq p$, 


$$
\left|D^{r}\left(f-\mathscr{T}_{a, p} f\right)(x)\right| \leq \frac{(b-a)^{p+1-r-1 / q}}{(p-r) !}\left\|D^{p+1} f\right\|_{L_{q}([a, b])},
$$

and

$$
\left\|D^{r}\left(f-\mathscr{T}_{a, p} f\right)\right\|_{L_{q}([a, b])} \leq \frac{(b-a)^{p+1-r}}{(p-r) !}\left\|D^{p+1} f\right\|_{L_{q}([a, b])} .
$$

Proof. By differentiating the integral form of the Taylor approximation error (5.6) and using the Hölder inequality (5.4), we obtain

$$
\begin{aligned}
\left|D^{r}\left(f-\mathscr{T}_{a, p} f\right)(x)\right| & =\frac{1}{(p-r) !} \int_{a}^{b}(x-y)_{+}^{p-r} D^{p+1} f(y) \mathrm{d} y \\
& \leq \frac{1}{(p-r) !}\left[\int_{a}^{b}(x-y)_{+}^{(p-r) q^{\prime}} \mathrm{d} y\right]^{1 / q^{\prime}}\left\|D^{p+1} f\right\|_{L_{q}([a, b])} \\
& \leq \frac{(b-a)^{p-r+1 / q^{\prime}}}{(p-r) !\left((p-r) q^{\prime}+1\right)^{1 / q^{\prime}}}\left\|D^{p+1} f\right\|_{L_{q}([a, b])} .
\end{aligned}
$$

Since $1 / q+1 / q^{\prime}=1$ and $(p-r) q^{\prime} \geq 0$, we obtain (5.7). Finally, taking the $L_{q}$-norm shows (5.8).

For the sake of simplicity one can use the following weaker, but simpler upper bound,

$$
\left\|D^{r}\left(f-\mathscr{T}_{a, p} f\right)\right\|_{L_{q}([a, b])} \leq(b-a)^{p+1-r}\left\|D^{p+1} f\right\|_{L_{q}([a, b])} .
$$

\subsection{Spline Quasi-Interpolation}

In general, a spline approximating a function $f$ can be written in terms of B-splines as

$$
\mathscr{Q} f(x):=\sum_{j=1}^{n} \lambda_{j}(f) B_{j, p, \boldsymbol{\xi}}(x)
$$

for suitable coefficients $\lambda_{j}(f)$. The spline in (5.10) will be referred to as a quasiinterpolant to $f$ whenever it provides a "reasonable" approximation to $f$.

Both interpolation and least squares are examples of quasi-interpolation methods. They are global methods since we have to solve an $n$ by $n$ system of linear equations to find their coefficients $\lambda_{j}(f)$. It follows that the value of the spline (5.10) at a point depends on all the data.

In this section we focus on local linear methods, i.e., methods where each $\lambda_{j}$ is a linear functional only depending on the values of $f$ in the support of $B_{j, p, \boldsymbol{\xi}}$. In principle, it suffices to be "near" the support of $B_{j, p, \xi}$, but we want to keep the presentation as simple as possible. In order to deal with point evaluator functionals we assume here that $f \in C^{-1}([a, b])$, where $[a, b]$ is a bounded interval. We consider a spline space $\mathbb{S}_{p, \xi}$, where the knot sequence $\boldsymbol{\xi}$ is $(p+1)$-basic and the basic interval $\left[\xi_{p+1}, \xi_{n+1}\right]$ is equal to $[a, b]$. 
With the aim of constructing a spline quasi-interpolant with optimal accuracy, we need to introduce some basic approximation properties of quasi-interpolants of the form (5.10). Since we are interested in local methods, we start with the following definition.

Definition 33. We say that a linear functional $\lambda: C^{-1}([a, b]) \rightarrow \mathbb{R}$ is supported on a nonempty set $\mathscr{S} \subset[a, b]$ if $\lambda(f)=0$ for any $f \in C^{-1}([a, b])$ which vanishes on $\mathscr{S}$.

Note that the set $\mathscr{S}$ in this definition is not uniquely defined and is not necessary minimal.

To construct our quasi-interpolant, we first require linear functionals that are supported on intervals consisting of a few knot intervals. This will ensure that $\mathscr{Q} f$ only depends locally on $f$. To ensure a good approximation power, we also require polynomial reproduction up to a given degree. Finally, to bound the error, a boundedness assumption on the linear functionals is needed. This leads to the following definitions.

Definition 34. The quasi-interpolant $\mathscr{Q}$ given by (5.10) is called a local quasiinterpolant if

(i) each $\lambda_{j}$ is supported on the interval $I_{j}$, where

$$
I_{j}:=\left[\xi_{j}, \xi_{j+p+1}\right] \cap[a, b],
$$

such that $I_{j}$ has nonempty interior;

(ii) the $\lambda_{j}$ are chosen so that $(5.10)$ reproduces $\mathbb{P}_{l}$, i.e.,

$$
\mathscr{Q} g(x)=g(x) \text { for all } x \in[a, b] \text { and all } g \in \mathbb{P}_{l},
$$

for some l with $0 \leq l \leq p$.

Definition 35. A local quasi-interpolant $\mathscr{Q}$ is called bounded in an $L_{q}$-norm, $1 \leq$ $q \leq \infty$, if there is a constant $C_{\mathscr{Q}}$ such that for each $\lambda_{j}$ we have

$$
\left|\lambda_{j}(f)\right| \leq C_{\mathscr{Q}} h_{j, p, \boldsymbol{\xi}}^{-1 / q}\|f\|_{L_{q}\left(I_{j}\right)} \text { for all } f \in C^{-1}\left(I_{j}\right),
$$

where

$$
h_{j, p, \boldsymbol{\xi}}:=\max _{\max (j, p+1) \leq k \leq \min (j+p, n)} \xi_{k+1}-\xi_{k} .
$$

Note that $h_{j, p, \xi}$ is the largest length of a knot interval in the intersection of the basic interval with the support of $B_{j, p, \xi}$. The requirement (5.11) ensures that the spline in (5.10) provides a local approximation to $f$. The polynomial reproduction as stated in (5.12) coupled with the boundedness of the linear functionals are the main ingredients to prove the approximation power of any bounded local quasiinterpolant.

We now give both a local and a global version of the approximation power of bounded local quasi-interpolants. To turn a local bound into a global bound we first state the following lemma. 
Lemma 36. Suppose that $f \in L_{q}\left(\left[\xi_{p+1}, \xi_{n+1}\right]\right)$ for some $q, 1 \leq q<\infty$, and that $m_{i_{1}}, \ldots, m_{i_{2}}$ are integers with $m_{i_{1}}<\cdots<m_{i_{2}}, \xi_{p+1} \leq \xi_{m_{i_{1}}}$ and $\xi_{m_{i_{2}}+k} \leq \xi_{n+1}$ for some positive integer $k$ and integers $i_{1} \leq i_{2}$. Then,

$$
\left(\sum_{j=i_{1}}^{i_{2}}\|f\|_{L_{q}\left(\left[\xi_{m_{j}}, \xi_{m_{j}+k}\right]\right)}^{q}\right)^{1 / q} \leq k^{1 / q}\|f\|_{L_{q}\left(\left[\xi_{p+1}, \xi_{n+1}\right]\right)} .
$$

Proof. Under the stated assumptions, each knot interval in $\left[\xi_{p+1}, \xi_{n+1}\right]$ is counted at most $k$ times and moreover all the local intervals $\left[\xi_{m_{j}}, \xi_{m_{j}+k}\right]$ are contained in $\left[\xi_{p+1}, \xi_{n+1}\right]$. The definition of the $L_{q}$-norm gives immediately (5.15).

Theorem 37 (Quasi-Interpolation Error). Let $\mathscr{Q}$ be a bounded local quasiinterpolant in an $L_{q}$-norm, $1 \leq q \leq \infty$, as in Definitions 34 and 35 . Let l, $p$ be integers with $0 \leq l \leq p$. Suppose $\xi_{m}<\xi_{m+1}$ for some $p+1 \leq m \leq n$, and let $f \in W_{q}^{l+1}\left(J_{m}\right)$ with

$$
J_{m}:=\left[\xi_{m-p}, \xi_{m+p+1}\right] \cap[a, b] .
$$

Then,

$$
\|f-\mathscr{Q} f\|_{L_{q}\left(\left[\xi_{m}, \xi_{m+1}\right]\right)} \leq \frac{(2 p+1)^{l+1}}{l !}\left(1+C_{\mathscr{Q}}\right) h_{m, \xi}^{l+1}\left\|D^{l+1} f\right\|_{L_{q}\left(J_{m}\right)},
$$

where $h_{m, \xi}$ is the largest length of a knot interval in $J_{m}$. Moreover, if $f \in W_{q}^{l+1}([a, b])$ then

$$
\|f-\mathscr{Q} f\|_{L_{q}([a, b])} \leq \frac{(2 p+1)^{l+1+1 / q}}{l !}\left(1+C_{\mathscr{Q}}\right) h_{\xi}^{l+1}\left\|D^{l+1} f\right\|_{L_{q}([a, b])},
$$

where

$$
h_{\boldsymbol{\xi}}:=\max _{p+1 \leq j \leq n} \xi_{j+1}-\xi_{j} .
$$

Proof. Note that $f$ is continuous since $l \geq 0$. Suppose $x \in\left[\xi_{m}, \xi_{m+1}\right)$. By the local partition of unity (2.21) and by (5.13) we have

$$
|\mathscr{Q} f(x)| \leq \max _{m-p \leq j \leq m}\left|\lambda_{j}(f)\right| \leq C_{\mathscr{Q}} \max _{m-p \leq j \leq m} h_{j, p, \xi}^{-1 / q}\|f\|_{L_{q}\left(I_{j}\right)} .
$$

Since $\xi_{m+1}-\xi_{m} \leq \min _{m-p \leq j \leq m} h_{j, p, \boldsymbol{\xi}}$ and $J_{m}=\cup_{m-p \leq j \leq m} I_{j}$ we find

$$
\|\mathscr{Q} f\|_{L_{q}\left(\left[\xi_{m}, \xi_{m+1}\right]\right)} \leq C_{\mathscr{Q}}\|f\|_{L_{q}\left(J_{m}\right)} .
$$

From (5.12) we know that $\mathscr{Q}$ reproduces any polynomial $g \in \mathbb{P}_{l}$, and so the triangle inequality gives

$$
\|f-\mathscr{Q} f\|_{L_{q}\left(\left[\xi_{m}, \xi_{m+1}\right]\right)} \leq\|f-g\|_{L_{q}\left(\left[\xi_{m}, \xi_{m+1}\right]\right)}+\|\mathscr{Q}(f-g)\|_{L_{q}\left(\left[\xi_{m}, \xi_{m+1}\right]\right)} .
$$

Since $\left[\xi_{m}, \xi_{m+1}\right] \subset J_{m}$ and by (5.18) for any $g \in \mathbb{P}_{l}$, we have

$$
\|f-\mathscr{Q} f\|_{L_{q}\left(\left[\xi_{m}, \xi_{m+1}\right]\right)} \leq\left(1+C_{\mathscr{Q}}\right)\|f-g\|_{L_{q}\left(J_{m}\right)} .
$$


Let us now choose $g:=\mathscr{T}_{\xi_{m-p}, l} f$, where $\mathscr{T}_{\xi_{m-p}, l} f$ is the Taylor polynomial of degree $l$ defined in (5.5) with $a=\xi_{m-p}$. Then, by (5.8) with $r=0$ we have

$$
\|f-g\|_{L_{q}\left(J_{m}\right)} \leq \frac{(2 p+1)^{l+1}}{l !} h_{m, \boldsymbol{\xi}}^{l+1}\left\|D^{l+1} f\right\|_{L_{q}\left(J_{m}\right)} .
$$

Combining the inequalities (5.19) and (5.20) gives the local bound.

Since each $J_{m}$ is contained in the basic interval $[a, b]$ the global bound follows immediately from the local one and Lemma 36.

Example 38. Let $\boldsymbol{\xi}$ be a $(p+1)$-open knot sequence for $p \geq 1$, and consider the operator

$$
\mathscr{V}_{p, \boldsymbol{\xi}} f(x):=\sum_{j=1}^{n} f\left(\xi_{j, p, \boldsymbol{\xi}}^{*}\right) B_{j, p, \boldsymbol{\xi}}(x),
$$

where $\xi_{j, p, \xi}^{*}$ is the $j$-th Greville point of degree $p$; see (3.21). This operator is known as the Schoenberg operator, and was introduced in [43, Section 10]. It is a bounded local quasi-interpolant in the $L_{\infty}$-norm with $l=1$ and $C_{\mathscr{Q}}=1$. We also know that $\xi_{j, p, \xi}^{*}$ belongs to $\left[\xi_{j+1}, \xi_{j+p}\right]$. Therefore, Theorem 37 implies for any $f \in W_{\infty}^{2}([a, b])$,

$$
\left\|f-\mathscr{V}_{p, \boldsymbol{\xi}} f\right\|_{L_{\infty}([a, b])} \leq 2(2 p+1)^{2} h_{\xi}^{2}\left\|D^{2} f\right\|_{L_{\infty}([a, b])} .
$$

The next proposition can be used to find the degree $l$ of polynomials reproduced by a linear quasi-interpolant.

Proposition 39. Let

$$
\left\{\varphi_{j, 0}, \ldots, \varphi_{j, l}\right\}, \quad j=1, \ldots, n, \quad 0 \leq l \leq p
$$

be $n$ sets of basis functions for $\mathbb{P}_{l}$, and let

$$
\varphi_{j, i}=\sum_{k=1}^{n} c_{j, i, k} B_{k, p, \xi}
$$

be their B-spline representations. The linear quasi-interpolant (5.10) reproduces $\mathbb{P}_{l}$ provided the corresponding linear functionals satisfy

$$
\lambda_{j}\left(\varphi_{j, i}\right)=c_{j, i, j}, \quad j=1, \ldots, n, \quad i=0, \ldots, l .
$$

Proof. Any $g \in \mathbb{P}_{l}$ can be written both in terms of the $\varphi$ 's and the B-splines, say

$$
g=\sum_{i=0}^{l} b_{j, i} \varphi_{j, i}=\sum_{k=1}^{n} b_{k} B_{k, p, \boldsymbol{\xi}}, \quad j=1, \ldots, n .
$$

By (5.24) and (5.26) for $j=1, \ldots, n$,

$$
g=\sum_{i=0}^{l} b_{j, i}\left(\sum_{k=1}^{n} c_{j, i, k} B_{k, p, \boldsymbol{\xi}}\right)=\sum_{k=1}^{n}\left(\sum_{i=0}^{l} b_{j, i} c_{j, i, k}\right) B_{k, p, \boldsymbol{\xi}}=\sum_{k=1}^{n} b_{k} B_{k, p, \boldsymbol{\xi}}
$$


By linear independence of the B-splines and choosing $j=k$ we obtain

$$
b_{k}=\sum_{i=0}^{l} b_{k, i} c_{k, i, k}
$$

Similarly, for $\mathscr{Q} g$ using (5.26) with $j=k$,

$$
\mathscr{Q} g:=\sum_{k=1}^{n} \lambda_{k}(g) B_{k, p, \xi}=\sum_{k=1}^{n} \lambda_{k}\left(\sum_{i=0}^{l} b_{k, i} \varphi_{k, i}\right) B_{k, p, \xi}
$$

From the linearity of $\lambda_{k}$ and (5.25), (5.27) and finally (5.26) again we obtain

$$
\mathscr{Q} g=\sum_{k=1}^{n} \sum_{i=0}^{l} b_{k, i} \lambda_{k}\left(\varphi_{k, i}\right) B_{k, p, \boldsymbol{\xi}}=\sum_{k=1}^{n} \sum_{i=0}^{l} b_{k, i} c_{k, i, k} B_{k, p, \boldsymbol{\xi}}=\sum_{k=1}^{n} b_{k} B_{k, p, \boldsymbol{\xi}}=g .
$$

The next proposition gives a sufficient condition for a quasi-interpolant to reproduce the whole spline space.

Proposition 40. The linear quasi-interpolant (5.10) reproduces the whole spline space, i.e.,

$$
\mathscr{Q} s(x)=s(x), \quad s \in \mathbb{S}_{p, \boldsymbol{\xi}}, \quad x \in\left[\xi_{p+1}, \xi_{n+1}\right],
$$

if $\mathscr{Q}$ reproduces $\mathbb{P}_{p}$ and each linear functional $\lambda_{j}$ is supported on one knot interval ${ }^{6}$

$$
\left[\xi_{m_{j}}^{+}, \xi_{m_{j}+1}^{-}\right] \subset\left[\xi_{j}, \xi_{j+p+1}\right], \text { with } \xi_{m_{j}}<\xi_{m_{j}+1} .
$$

In other words, $\mathscr{Q}$ is a projector onto the spline space $\mathbb{S}_{p, \xi}$.

Proof. Let $j$ with $1 \leq j \leq n$ be fixed. By the linearity it suffices to prove that

$$
\lambda_{j}\left(B_{i, p, \xi}\right)=\delta_{i, j}, \quad i=1, \ldots, n,
$$

where $\delta_{i, j}$ stands for the classical Kronecker delta. On the interval $\left[\xi_{m_{j}}^{+}, \xi_{m_{j}+1}^{-}\right]$the local support property implies that $\lambda_{j}\left(B_{i, p, \boldsymbol{\xi}}\right)=0$ for $i \notin\left\{m_{j}-p, \ldots, m_{j}\right\}$. This follows because we use the left limit at $\xi_{m_{j}+1}$ if necessary. Since $B_{i, p, \xi} \in \mathbb{P}_{p}$ on this interval, we have

$$
B_{i, p, \boldsymbol{\xi}}(x)=\mathscr{Q}\left(B_{i, p, \boldsymbol{\xi}}\right)(x)=\sum_{k=m_{j}-p}^{m_{j}} \lambda_{k}\left(B_{i, p, \boldsymbol{\xi}}\right) B_{k, p, \boldsymbol{\xi}}(x), \quad x \in\left[\xi_{m_{j}}, \xi_{m_{j}+1}\right),
$$

and by local linear independence of the B-splines we obtain $\lambda_{k}\left(B_{i, p, \xi}\right)=\delta_{k, i}$ for $k=m_{j}-p, \ldots, m_{j}$. In particular, it holds for $k=i$ since the condition (5.29) implies that $m_{j}-p \leq j \leq m_{j}$.

${ }^{6}$ This notation means that if $\lambda_{j}(f)$ uses the value of $f$ or one of its derivatives at $\xi_{m_{j}}$ (or $\xi_{m_{j}+1}$ ) then this value is obtained by taking the one sided limit from the right (or the left). 
Example 41. Let $p=2$, and let $\boldsymbol{\xi}$ be a 3-open knot sequence with at most double knots in the interior. We consider the operator

$$
\mathscr{Q}_{2, \boldsymbol{\xi}} f(x):=\sum_{j=1}^{n}\left(a_{2,0} f\left(\xi_{j+1}\right)+a_{2,1} f\left(\xi_{j, 2, \boldsymbol{\xi}}^{*}\right)+a_{2,2} f\left(\xi_{j+2}\right)\right) B_{j, 2, \boldsymbol{\xi}}(x),
$$

where $\xi_{j, 2, \boldsymbol{\xi}}^{*}=\left(\xi_{j+1}+\xi_{j+2}\right) / 2$ is the $j$-th Greville point of degree 2. From Example 24 we know that if we choose $a_{2,0}=a_{2,2}=-1 / 2$ and $a_{2,1}=2$ then $\mathscr{Q}_{2, \xi}$ reproduces $\mathbb{P}_{2}$, i.e., $l=2$. Proposition 40 says that it is even a projector on the spline space $\mathbb{S}_{2, \xi}$. Moreover,

$$
\left|-\frac{1}{2} f\left(\xi_{j+1}\right)+2 f\left(\xi_{j, 2, \xi}^{*}\right)-\frac{1}{2} f\left(\xi_{j+2}\right)\right| \leq 3\|f\|_{L_{\infty}\left(\left[\xi_{j}, \xi_{j+3}\right]\right)} .
$$

It follows that $\mathscr{Q}_{2, \xi}$ is a bounded local quasi-interpolant in the $L_{\infty}$-norm with $l=2$ and $C_{\mathscr{Q}}=3$. In this case, Theorem 37 implies for any $f \in W_{\infty}^{3}([a, b])$,

$$
\left\|f-\mathscr{Q}_{2, \xi} f\right\|_{L_{\infty}([a, b])} \leq 4 \frac{5^{3}}{2 !} h_{\xi}^{3}\left\|D^{3} f\right\|_{L_{\infty}([a, b])}
$$

showing that the error is $O\left(h_{\xi}^{3}\right)$.

\subsection{Approximation Power of Splines}

In this section we want to understand how well a function can be approximated by a spline. In order words, we want to investigate the distance between a general function $f$ and the piecewise polynomial space $\mathbb{S}_{p}^{r}(\Delta)$ defined in (3.12). From Theorem 15 we know that $\mathbb{S}_{p}^{r}(\Delta)=\mathbb{S}_{p, \xi}$ for a suitable choice of the knot sequence $\boldsymbol{\xi}:=\left\{\xi_{i}\right\}_{i=1}^{n+p+1}$. In particular, $\boldsymbol{\xi}$ can be chosen to be $(p+1)$-open. Therefore, without loss of generality, we consider the distance between a general function $f$ and the spline space $\mathbb{S}_{p, \boldsymbol{\xi}}$ of degree $p$ over the $(p+1)$-open knot sequence $\boldsymbol{\xi}$. For a given $f \in L_{q}\left(\left[\xi_{p+1}, \xi_{n+1}\right]\right)$ with $1 \leq q \leq \infty$, we define

$$
\operatorname{dist}_{q}\left(f, \mathbb{S}_{p, \boldsymbol{\xi}}\right):=\inf _{s \in \mathbb{S}_{p, \boldsymbol{\xi}}}\|f-s\|_{L_{q}\left(\left[\xi_{p+1}, \xi_{n+1}\right]\right)} .
$$

We are also interested in estimates for the distance between derivatives of $f$ and derivative spline spaces. To this end, in this section we use the simplified notation $D^{r} s:=D_{+}^{r} s$ for the derivatives of a spline $s \in \mathbb{S}_{p, \xi}$ with the usual convention of left continuity at the right endpoint of the basic interval. Note that with such a notation we ensure that $D^{r} s(x)$ exists for all $x$. In the same spirit, we use the notation $D^{r} \mathbb{S}_{p, \boldsymbol{\xi}}:=D_{+}^{r} \mathbb{S}_{p, \boldsymbol{\xi}}$ for the $r$-th derivative spline space. We recall from Section 3.2 that this derivative space is a piecewise polynomial space of degree $p-r$ with a certain smoothness, i.e.,

$$
\mathbb{S}_{p-r}^{r-r}(\Delta)=D^{r} \mathbb{S}_{p, \boldsymbol{\xi}}
$$

where the partition $\Delta$ consists of the distinct break points in the knot sequence $\boldsymbol{\xi}$ and the smoothness $\boldsymbol{r}$ is related to the multiplicity of the knots, according to the rule 
in (3.5). This leads to the following more general definition of distance. For a given $f \in W_{q}^{r}\left(\left[\xi_{p+1}, \xi_{n+1}\right]\right)$ with $1 \leq q \leq \infty$ and $0 \leq r \leq p$, we define

$$
\operatorname{dist}_{q}\left(D^{r} f, D^{r} \mathbb{S}_{p, \boldsymbol{\xi}}\right):=\inf _{s \in \mathbb{S}_{p, \boldsymbol{\xi}}}\left\|D^{r}(f-s)\right\|_{L_{q}\left(\left[\xi_{p+1}, \xi_{n+1}\right]\right)} .
$$

We will derive the following upper bound for $\operatorname{dist}_{q}\left(D^{r} f, D^{r} \mathbb{S}_{p, \boldsymbol{\xi}}\right)$.

Theorem 42 (Distance to a Function). For any $0 \leq r \leq l \leq p$ and $f \in W_{q}^{l+1}\left(\left[\xi_{p+1}, \xi_{n+1}\right]\right)$ with $1 \leq q \leq \infty$ we have

$$
\operatorname{dist}_{q}\left(D^{r} f, D^{r} \mathbb{S}_{p, \boldsymbol{\xi}}\right) \leq K\left(h_{\boldsymbol{\xi}}\right)^{l+1-r}\left\|D^{l+1} f\right\|_{L_{q}\left(\left[\xi_{p+1}, \xi_{n+1}\right]\right)},
$$

where $h_{\boldsymbol{\xi}}:=\max _{p+1 \leq j \leq n}\left(\xi_{j+1}-\xi_{j}\right)$ and $K$ is a constant depending only on $p$.

The distance result will be shown by explicitly constructing a suitable spline quasi-interpolant which achieves this order of approximation; see Theorem 49. For sufficiently smooth $f$, the upper bound behaves like $\left(h_{\boldsymbol{\xi}}\right)^{p+1-r}$.

\subsubsection{A Spline Quasi-Interpolant}

Given an integer $p \geq 0$ and a $(p+1)$-open knot sequence $\boldsymbol{\xi}$, we define a specific spline approximant of degree $p$ over $\boldsymbol{\xi}$ to a given function $f$. Let $\left[\xi_{m_{j, p}}, \xi_{m_{j, p}+1}\right]$ be a knot interval of largest length in $\left[\xi_{j}, \xi_{j+p+1}\right]$ for any $j=1, \ldots, n$ and $h_{j, p, \boldsymbol{\xi}}:=$ $\xi_{m_{j, p}+1}-\xi_{m_{j, p}}>0$. The spline approximant to $f$ is constructed as

$$
\mathscr{Q}_{p, \boldsymbol{\xi}} f(x):=\sum_{j=1}^{n} \mathscr{L}_{j, p, \boldsymbol{\xi}}(f) B_{j, p, \boldsymbol{\xi}}(x)
$$

where

$$
\mathscr{L}_{j, p, \boldsymbol{\xi}}(f):=\frac{1}{h_{j, p, \boldsymbol{\xi}}} \int_{\xi_{m_{j, p}}}^{\xi_{m_{j, p}+1}}\left(\sum_{i=0}^{p} a_{j, i}\left(\frac{x-\xi_{m_{j, p}}}{h_{j, p, \boldsymbol{\xi}}}\right)^{i}\right) f(x) \mathrm{d} x
$$

and the coefficients $a_{j, i}, i=0, \ldots, p$ are such that

$$
\mathscr{L}_{j, p, \boldsymbol{\xi}}\left(\left(\frac{x-\xi_{m_{j, p}}}{h_{j, p, \boldsymbol{\xi}}}\right)^{i}\right)=c_{j, i, j}, \quad i=0, \ldots, p,
$$

where

$$
\left(\frac{x-\xi_{m_{j, p}}}{h_{j, p, \boldsymbol{\xi}}}\right)^{i}=\sum_{k=m_{j, p}-p}^{m_{j, p}} c_{j, i, k} B_{k, p, \boldsymbol{\xi}}(x), \quad x \in\left[\xi_{m_{j, p}}, \xi_{m_{j, p}+1}\right), \quad i=0, \ldots, p .
$$

In the next lemmas we collect some properties for the spline approximation (5.32). 
Lemma 43. The above spline approximation is well defined and reproduces polynomials, i.e., for any polynomial $g \in \mathbb{P}_{p}$ we have

$$
\mathscr{Q}_{p, \xi} g(x)=g(x), \quad x \in\left[\xi_{p+1}, \xi_{n+1}\right] .
$$

Moreover, it is a projector onto the spline space $\mathbb{S}_{p, \xi}$, i.e., for any spline $s \in \mathbb{S}_{p, \boldsymbol{\xi}}$ we have

$$
\mathscr{Q}_{p, \xi} s(x)=s(x), \quad x \in\left[\xi_{p+1}, \xi_{n+1}\right]
$$

and, in particular,

$$
s(x)=\sum_{j=1}^{n} \mathscr{L}_{j, p, \boldsymbol{\xi}}(s) B_{j, p, \boldsymbol{\xi}}(x), \quad x \in\left[\xi_{p+1}, \xi_{n+1}\right]
$$

Proof. By applying $\mathscr{L}_{j, p, \boldsymbol{\xi}}$ to the polynomials $\left(\frac{x-\xi_{m_{j, p}}}{h_{j, p, \boldsymbol{\xi}}}\right)^{r}, r=0, \ldots, p$, the coefficients $a_{j, i}$ are given by the solution of the linear system

$$
H_{p+1} \boldsymbol{a}_{j}=\boldsymbol{c}_{j}
$$

where $\boldsymbol{a}_{j}:=\left(a_{j, 0}, \ldots, a_{j, p}\right)^{T}, \boldsymbol{c}_{j}:=\left(c_{j, 0, j}, \ldots, c_{j, p, j}\right)^{T}$, and $H_{p+1}$ is a $(p+1) \times(p+$ 1) matrix with elements

$$
\left(H_{p+1}\right)_{i+1, r+1}:=\frac{1}{h_{j, p, \boldsymbol{\xi}}} \int_{\xi_{m_{j, p}}}^{\xi_{m_{j, p}+1}}\left(\frac{x-\xi_{m_{j, p}}}{h_{j, p, \boldsymbol{\xi}}}\right)^{r+i} \mathrm{~d} x=\frac{1}{i+r+1}, \quad i, r=0, \ldots, p .
$$

This is the well-known Hilbert matrix which is nonsingular and it follows that the spline approximation (5.32) is well defined. From Proposition 39 we deduce that (5.36) holds.

Since we only integrate over one subinterval when we define $\mathscr{L}_{j, p, \xi}$, we conclude that it reproduces not only polynomials but also splines, and (5.37) follows from Proposition 40.

Lemma 44. For $p \geq 0$ and $1 \leq q \leq \infty$ we have for any $f \in L_{q}\left(\left[\xi_{m_{j, p}}, \xi_{m_{j, p}+1}\right]\right)$,

$$
\left|\mathscr{L}_{j, p, \boldsymbol{\xi}}(f)\right| \leq C h_{j, p, \boldsymbol{\xi}}^{-1 / q}\|f\|_{L_{q}\left(\left[\xi_{m_{j, p}}, \xi_{m_{j, p}+1}\right]\right)}, \quad j=1, \ldots, n,
$$

where $C$ is a constant depending only on $p$.

Proof. By (2.20), (2.10) and (2.13) we have

$$
\left|c_{j, i, j}\right|=\frac{i !}{p !}\left|\frac{D^{p-i} \psi_{j, p, \boldsymbol{\xi}}\left(\xi_{m_{j, p}}\right)}{h_{j, p, \boldsymbol{\xi}}^{i}}\right| \leq\left(\frac{\xi_{j+p+1}-\xi_{j}}{h_{j, p, \boldsymbol{\xi}}}\right)^{i} \leq(p+1)^{i}, \quad i=0, \ldots, p .
$$

Here we used that $\left[\xi_{m_{j, p}}, \xi_{m_{j, p}+1}\right]$ is a knot interval of largest length in $\left[\xi_{j}, \xi_{j+p+1}\right]$. Since $0 \leq \frac{x-\xi_{m_{j, p}}}{h_{j, p, \boldsymbol{\xi}}} \leq 1$ for $x \in\left[\xi_{m_{j, p}}, \xi_{m_{j, p}+1}\right]$, we get from (5.33), 


$$
\begin{aligned}
\left|\mathscr{L}_{j, p, \boldsymbol{\xi}}(f)\right| & \leq(p+1) h_{j, p, \boldsymbol{\xi}}^{-1}\left\|\boldsymbol{a}_{j}\right\|_{\infty}\|f\|_{L_{1}\left(\left[\xi_{m_{j, p},}, \xi_{m_{j, p}+1}\right]\right)} \\
& \leq(p+1) h_{j, p, \boldsymbol{\xi}}^{-1}\left\|H_{p+1}^{-1}\right\|_{\infty}\left\|\boldsymbol{c}_{j}\right\|_{\infty}\|f\|_{L_{1}\left(\left[\xi_{m_{j, p}}, \xi_{m_{j, p}+1}\right]\right)} .
\end{aligned}
$$

This gives $\left|\mathscr{L}_{j, p, \boldsymbol{\xi}}(f)\right| \leq C h_{j, p, \boldsymbol{\xi}}^{-1}\|f\|_{L_{1}\left(\left[\xi_{m_{j, p},}, \xi_{m_{j, p}+1}\right]\right)}$, where $C:=\left\|H_{p+1}^{-1}\right\|_{\infty}(p+1)^{p+1}$ only depends on $p$. By the Hölder inequality (5.4) we arrive at (5.40).

We now give a bound for the derivative of $\mathscr{Q}_{p, \xi} f$. To this end, we recall from (2.25) that

$$
\Delta_{m, k}:=\min _{m-k+1 \leq i \leq m} h_{i, k}, \quad h_{i, k}:=\xi_{i+k}-\xi_{i}, \quad k=1, \ldots, p,
$$

and that $\Delta_{m, k}>0$ for all $k$ if $\xi_{m}<\xi_{m+1}$.

Lemma 45. Suppose $\xi_{m}<\xi_{m+1}$ for some $p+1 \leq m \leq n$, and let $f \in L_{q}\left(\left[\xi_{m-p}, \xi_{m+p+1}\right]\right)$ with $1 \leq q \leq \infty$. Then, we have for $0 \leq r \leq p$,

$$
\left\|D^{r}\left(\mathscr{Q}_{p, \xi} f\right)\right\|_{L_{q}\left(\left[\xi_{m}, \xi_{m+1}\right]\right)} \leq C\left(\prod_{k=p-r+1}^{p} \frac{1}{\Delta_{m, k}}\right)\|f\|_{L_{q}\left(\left[\xi_{m-p}, \xi_{m+p+1}\right]\right)}
$$

where $\Delta_{m, k}$ is defined in (2.25) and $C$ is a constant depending only on $p$.

Proof. From the quasi-interpolant definition (5.32), the local support property (3.6) and Lemma 44, we have for $x \in\left[\xi_{m}, \xi_{m+1}\right)$,

$$
\begin{aligned}
& \left|D^{r}\left(\mathscr{Q}_{p, \boldsymbol{\xi}} f\right)(x)\right|=\left|\sum_{j=m-p}^{m} \mathscr{L}_{j, p, \boldsymbol{\xi}}(f) D^{r} B_{j, p, \boldsymbol{\xi}}(x)\right| \\
& \quad \leq \max _{m-p \leq j \leq m}\left|D^{r} B_{j, p, \boldsymbol{\xi}}(x)\right| \sum_{j=m-p}^{m}\left|\mathscr{L}_{j, p, \boldsymbol{\xi}}(f)\right| \\
& \leq(p+1) \max _{m-p \leq j \leq m}\left|D^{r} B_{j, p, \boldsymbol{\xi}}(x)\right| \max _{m-p \leq j \leq m} h_{j, p, \boldsymbol{\xi}}^{-1 / q}\|f\|_{L_{q}\left(\left[\xi_{m-p}, \xi_{m+p+1}\right]\right)} .
\end{aligned}
$$

Note that $\left[\xi_{m}, \xi_{m+1}\right] \subset\left[\xi_{j}, \xi_{j+p+1}\right]$ for $j=m-p, \ldots, m$. Since $h_{j, p, \xi}$ is the length of the largest knot interval in $\left[\xi_{j}, \xi_{j+p+1}\right]$, we have $\xi_{m+1}-\xi_{m} \leq h_{j, p, \xi}$ for $j=m-$ $p, \ldots, m$. Replacing $\left|D^{r} B_{j, p, \boldsymbol{\xi}}(x)\right|$ by the upper bound given in Proposition 11 and taking the $L_{q}$-norm result in (5.41).

The next lemma will complete the proof of Theorem 26 related to the condition number. Note that $\left[\xi_{p+1}, \xi_{n+1}\right]=\left[\xi_{1}, \xi_{n+p+1}\right]$ because the knot sequence $\boldsymbol{\xi}$ is open.

Lemma 46. For any $p \geq 0$, there exists a positive constant $K_{p}$ depending only on $p$, such that for any vector $\boldsymbol{c}:=\left(c_{1}, \ldots, c_{n}\right)$ and for any $1 \leq q \leq \infty$ we have

$$
\|\boldsymbol{c}\|_{q} \leq K_{p}\left\|\sum_{j=1}^{n} c_{j} N_{j, p, q, \boldsymbol{\xi}}\right\|_{L_{\left.q\left(\xi_{p+1}, \xi_{n+1}\right]\right)}},
$$

where $N_{j, p, q, \boldsymbol{\xi}}:=\gamma_{j, p, \boldsymbol{\xi}}^{-1 / q} B_{j, p, \boldsymbol{\xi}}$ and $\gamma_{j, p, \boldsymbol{\xi}}:=\left(\xi_{j+p+1}-\xi_{j}\right) /(p+1)$. 
Proof. Let $s:=\sum_{j=1}^{n} \gamma_{j, p, \xi}^{-1 / q} c_{j} B_{j, p, \xi}$. Observe that (5.38) and (5.40) imply

$$
\left|\gamma_{j, p, \boldsymbol{\xi}}^{-1 / q} c_{j}\right|=\left|\mathscr{L}_{j, p, \boldsymbol{\xi}}(s)\right| \leq C h_{j, p, \boldsymbol{\xi}}^{-1 / q}\|s\|_{L_{q}\left(\left[\xi_{m_{j, p}}, \xi_{m_{j, p}+1}\right]\right)} .
$$

Since $\gamma_{j, p, \boldsymbol{\xi}} / h_{j, p, \boldsymbol{\xi}} \leq 1$ we obtain

$$
\left|c_{j}\right| \leq C\|s\|_{L_{q}\left(\left[\xi_{m_{j, p}}, \xi_{m_{j, p}+1}\right]\right)} \leq C\|s\|_{L_{q}\left(\left[\xi_{j}, \xi_{j+p+1}\right]\right)} .
$$

Raising both sides to the $q$-th power and summing over $j$ gives

$$
\sum_{j=1}^{n}\left|c_{j}\right|^{q} \leq C^{q} \sum_{j=1}^{n} \int_{\xi_{j}}^{\xi_{j+p+1}}|s(x)|^{q} \mathrm{~d} x \leq(p+1) C^{q}\|s\|_{L_{q}\left(\left[\xi_{p+1}, \xi_{n+1}\right]\right)^{q}}^{q}
$$

When taking the $q$-th roots on both sides, we arrive at the inequality in (5.42) with $K_{p}:=(p+1) C \geq(p+1)^{1 / q} C$, which only depends on $p$.

\subsubsection{Distance to a Function}

The quasi-interpolant $\mathscr{Q}_{p, \xi} f$ described in the previous section can be used to obtain an upper bound for the distance between a given function $f$ and the spline space $\mathbb{S}_{p, \boldsymbol{\xi}}$ for $p \geq 0, n>p+1$ and $\boldsymbol{\xi}:=\left\{\xi_{j}\right\}_{j=1}^{n+p+1}$; see Theorem 49 . We recall that the knot sequence $\boldsymbol{\xi}$ is $(p+1)$-open. We start by giving a local and global upper bound for (the derivatives of) the difference between $f$ and $\mathscr{Q}_{p, \xi} f$.

Proposition 47. Suppose $\xi_{m}<\xi_{m+1}$ for some $p+1 \leq m \leq n$, and let $f \in W_{q}^{l+1}\left(\left[\xi_{m-p}, \xi_{m+p+1}\right]\right)$ with $0 \leq l \leq p$ and $1 \leq q \leq \infty$. If $\mathscr{Q}_{p, \boldsymbol{\xi}} f$ is defined as in (5.32), then we have for any $0 \leq r \leq l$,

$$
\left\|D^{r}\left(f-\mathscr{Q}_{p, \boldsymbol{\xi}} f\right)\right\|_{L_{q}\left(\left[\xi_{m}, \xi_{m+1}\right]\right)} \leq K_{m}\left(\xi_{m+p+1}-\xi_{m-p}\right)^{l+1-r}\left\|D^{l+1} f\right\|_{L_{q}\left(\left[\xi_{m-p}, \xi_{m+p+1}\right]\right)} .
$$

Here,

$$
K_{m}:=1+C \prod_{k=p-r+1}^{p} \frac{\xi_{m+p+1}-\xi_{m-p}}{\Delta_{m, k}},
$$

$\Delta_{m, k}$ is defined in (2.25) and $C$ is a constant depending only on $p$.

Proof. From Lemma 43 we know that $\mathscr{Q}_{p, \xi}$ reproduces any polynomial in $\mathbb{P}_{l}$, and so the triangle inequality gives

$$
\begin{aligned}
& \left\|D^{r}\left(f-\mathscr{Q}_{p, \boldsymbol{\xi}} f\right)\right\|_{L_{q}\left(\left[\xi_{m}, \xi_{m+1}\right]\right)} \\
& \quad \leq\left\|D^{r}(f-g)\right\|_{L_{q}\left(\left[\xi_{m}, \xi_{m+1}\right]\right)}+\left\|D^{r} \mathscr{Q}_{p, \boldsymbol{\xi}}(f-g)\right\|_{L_{q}\left(\left[\xi_{m}, \xi_{m+1}\right]\right)},
\end{aligned}
$$

for any $g \in \mathbb{P}_{l}$. Let us now set $g:=\mathscr{T}_{\xi_{m}, l} f$, where $\mathscr{T}_{\xi_{m}, l} f$ is the Taylor polynomial of degree $l$ defined in (5.5) with $a=\xi_{m}, b=\xi_{m+1}$. Then, Theorem 32 implies

$$
\left\|D^{r}(f-g)\right\|_{L_{q}\left(\left[\xi_{m}, \xi_{m+1}\right]\right)} \leq\left(\xi_{m+1}-\xi_{m}\right)^{l+1-r}\left\|D^{l+1} f\right\|_{L_{q}\left(\left[\xi_{m}, \xi_{m+1}\right]\right)} .
$$


On the other hand, since $f-g \in L_{q}\left(\left[\xi_{m-p}, \xi_{m+p+1}\right]\right)$, it follows from Lemma 45 that

$$
\left\|D^{r} \mathscr{Q}_{p, \boldsymbol{\xi}}(f-g)\right\|_{L_{q}\left(\left[\xi_{m}, \xi_{m+1}\right]\right)} \leq C\left(\prod_{k=p-r+1}^{p} \frac{1}{\Delta_{m, k}}\right)\|f-g\|_{L_{q}\left(\left[\xi_{m-p}, \xi_{m+p+1}\right]\right)},
$$

where $C$ is a constant depending only on $p$. Combining the above three inequalities gives the result.

We know that the ratio $\frac{\xi_{m+p+1}-\xi_{m-p}}{\Delta_{m, k}}$ is well defined because $\Delta_{m, k}>0$. For a uniform knot sequence

$$
\frac{\xi_{m+p+1}-\xi_{m-p}}{\Delta_{m, k}}=\frac{2 p+1}{k} .
$$

For a general knot sequence it is related to the "local mesh ratio", i.e., the ratio between the lengths of the largest and smallest knot intervals in a neighborhood of $\xi_{m}$.

The local error bound in Proposition 47 can be turned into a global one as in the following proposition.

Proposition 48. Let $f \in W_{q}^{l+1}\left(\left[\xi_{p+1}, \xi_{n+1}\right]\right)$ with $0 \leq l \leq p$ and $1 \leq q \leq \infty$. If $\mathscr{Q}_{p, \boldsymbol{\xi}} f$ is defined as in (5.32) then, for any $0 \leq r \leq l$,

$$
\left\|D^{r}\left(f-\mathscr{Q}_{p, \boldsymbol{\xi}} f\right)\right\|_{L_{q}\left(\left[\xi_{p+1}, \xi_{n+1}\right]\right)} \leq K h_{\xi}^{l+1-r}\left\|D^{l+1} f\right\|_{L_{q}\left(\left[\xi_{p+1}, \xi_{n+1}\right]\right)},
$$

where $h_{\boldsymbol{\xi}}:=\max _{p+1 \leq j \leq n}\left(\xi_{j+1}-\xi_{j}\right)$, and

$$
K:=(2 p+1)^{l+2-r}\left[1+C \max _{p+1 \leq m \leq n} \prod_{k=p-r+1}^{p} \frac{\xi_{m+p+1}-\xi_{m-p}}{\Delta_{m, k}}\right],
$$

where $\Delta_{m, k}$ is defined in (2.25) and $C$ is a constant depending only on $p$.

Proof. For $q=\infty$ the result follows immediately from Proposition 47 by taking into account that $\boldsymbol{\xi}$ is $(p+1)$-open. We now assume $1 \leq q<\infty$. Since

$$
\max _{p+1 \leq m \leq n}\left(\xi_{m+p+1}-\xi_{m-p}\right) \leq(2 p+1) h_{\boldsymbol{\xi}},
$$

the result follows from Lemma 36 and the local error bound in Proposition 47.

The expression $K$ in the upper bound in Proposition 48 depends on the position of the knots for $r>0$. However, for any knot sequence $\xi$, it is possible to construct a coarser knot sequence $\xi^{\sharp}$ such that the corresponding $K$ only depends on $p$. This can be obtained by a clever thinning process. The idea of thinning out a knot sequence to get a quasi-uniform sequence is credited to [47]; see [45, Section 6.4] for details. Since $\boldsymbol{\xi}^{\sharp}$ is a subsequence of $\boldsymbol{\xi}$, we have that $\mathbb{S}_{p, \boldsymbol{\xi}^{\sharp}}$ is a subspace of $\mathbb{S}_{p, \boldsymbol{\xi}}$. In particular, for any $f \in L_{q}\left(\left[\xi_{p+1}, \xi_{n+1}\right]\right)$ the spline approximation

$$
s_{p}:=\mathscr{Q}_{p, \xi^{\sharp}} f
$$


as defined in (5.32) belongs to the spline space $\mathbb{S}_{p, \boldsymbol{\xi}}$. This spline quasi-interpolant leads to the following important result.

Theorem 49 (Approximation Error). Let $f \in W_{q}^{l+1}\left(\left[\xi_{p+1}, \xi_{n+1}\right]\right)$ with $1 \leq q \leq \infty$ and $0 \leq l \leq p$. Then, there exists $s_{p} \in \mathbb{S}_{p, \boldsymbol{\xi}}$ such that

$$
\left\|D^{r}\left(f-s_{p}\right)\right\|_{L_{q}\left(\left[\xi_{p+1}, \xi_{n+1}\right]\right)} \leq K h_{\xi}^{l+1-r}\left\|D^{l+1} f\right\|_{L_{q}\left(\left[\xi_{p+1}, \xi_{n+1}\right]\right),} \quad 0 \leq r \leq l,
$$

where $h_{\boldsymbol{\xi}}:=\max _{p+1 \leq j \leq n}\left(\xi_{j+1}-\xi_{j}\right)$ and $K$ is a constant depending only on $p$.

The constant $K$ in Theorem 49 grows exponentially with $p$. However, this dependency on $p$ can be removed in some cases; see [1, Theorem 2] and [52, Theorem 7] for details. Theorem 49 immediately leads to the distance result in Theorem 42 .

\section{Hierarchical Splines and the Truncation Mechanism}

The hierarchical spline model is a simple strategy to mix locally spline spaces of different resolution (different mesh size and/or different degree). Hierarchical spline representations are defined in terms of a sequence of nested B-spline bases and a hierarchy of locally refined domains. In this section we define such hierarchical splines and focus on a set of basis functions with properties similar to B-splines.

\subsection{Hierarchical B-Splines}

Let $I$ be a closed interval of the real line, and consider a sequence of strictly nested spline spaces defined on $I$, say

$$
\mathbb{S}_{p_{1}, \boldsymbol{\xi}_{1}} \subset \mathbb{S}_{p_{2}, \boldsymbol{\xi}_{2}} \subset \cdots \subset \mathbb{S}_{p_{L}, \boldsymbol{\xi}_{L}}
$$

We assume that each knot sequence involved in (6.1),

$$
\boldsymbol{\xi}_{\ell}:=\left\{\xi_{1, \ell} \leq \xi_{2, \ell} \leq \cdots \leq \xi_{n_{\ell}+p_{\ell}+1, \ell}\right\}, \quad \ell=1, \ldots, L,
$$

is $(p+1)$-basic with basic interval $I$. Nestedness of the spaces is ensured if and only if

$$
0 \leq p_{\ell+1}-p_{\ell} \leq \mu_{\boldsymbol{\xi}_{\ell+1}}(\xi)-\mu_{\boldsymbol{\xi}_{\ell}}(\xi), \quad \xi \in \boldsymbol{\xi}_{\ell} \cap I, \quad \ell=1, \ldots, L-1 .
$$

Note that (6.2) implies that $\boldsymbol{\xi}_{\ell} \subseteq \boldsymbol{\xi}_{\ell+1}$. The assumption of dealing with $(p+1)$-basic knot sequences ensures that the corresponding $n_{\ell} \mathrm{B}$-splines are linearly independent on $I$. We denote the B-spline basis of the space $\mathbb{S}_{p_{\ell}, \xi_{\ell}}$ by

$$
\mathscr{B}_{\ell}:=\left\{B_{j, \ell}:=B_{j, p_{\ell}, \boldsymbol{\xi}_{\ell}}, j=1, \ldots, n_{\ell}\right\} .
$$


Next, consider a sequence of nested, closed subsets of $I$,

$$
I \supseteq \Omega_{1} \supseteq \Omega_{2} \supseteq \cdots \supseteq \Omega_{L},
$$

where $\Omega_{\ell}$ is the union of some closed knot intervals related to the knot sequence $\boldsymbol{\xi}_{\ell}$. Each $\Omega_{\ell}$ is not necessarily a single interval, but may consist of different disconnected intervals. We assume that each disconnected interval of $\Omega_{1}$ has nonempty interior. The collection of those subsets in (6.4) is denoted by

$$
\boldsymbol{\Omega}:=\left\{\Omega_{1}, \Omega_{2}, \ldots, \Omega_{L}\right\},
$$

and will be simply referred to as the domain hierarchy in $I$. We also set $\Omega_{L+1}:=\emptyset$. Finally, for a given function $f$ on $I$, we define its support on $\boldsymbol{\Omega}$ as

$$
\operatorname{supp}_{\Omega}(f):=\operatorname{supp}(f) \cap \Omega_{1} .
$$

Given a sequence of spline spaces and bases as in (6.1)-(6.3) and a domain hierarchy as in (6.4)-(6.5), we construct the corresponding set of hierarchical B-splines (in short, HB-splines) as follows ${ }^{7}$.

Definition 50. Given a domain hierarchy $\boldsymbol{\Omega}$, the corresponding set of $\mathbf{H B}$-splines is denoted by $\mathscr{H}_{\boldsymbol{\Omega}}$ and defined recursively as follows:

(i) $\mathscr{H}_{1}:=\left\{B_{j, 1} \in \mathscr{B}_{1}: \operatorname{supp}_{\Omega}\left(B_{j, 1}\right) \neq \emptyset\right\}$;

(ii) for $\ell=2, \ldots, L$ :

$$
\mathscr{H}_{\ell}:=\mathscr{H}_{\ell}^{C} \cup \mathscr{H}_{\ell}^{F},
$$

where

$$
\begin{aligned}
\mathscr{H}_{\ell}^{C} & :=\left\{B_{j, k} \in \mathscr{H}_{\ell-1}: \operatorname{supp}_{\boldsymbol{\Omega}}\left(B_{j, k}\right) \not \subset \Omega_{\ell}\right\} \\
\mathscr{H}_{\ell}^{F} & :=\left\{B_{j, \ell} \in \mathscr{B}_{\ell}: \operatorname{supp}_{\boldsymbol{\Omega}}\left(B_{j, \ell} \subseteq \Omega_{\ell}\right\}\right.
\end{aligned}
$$

(iii) $\mathscr{H}_{\boldsymbol{\Omega}}:=\mathscr{H}_{L}$.

To obtain the set of HB-splines, we first take all the B-splines in $\mathscr{B}_{1}$ whose support overlaps $\Omega_{1}$. Then, we apply a recursive procedure which selects at each level $\ell$ all the B-splines obtained in the previous step whose support is not entirely contained in $\Omega_{\ell}$ and all the B-splines in $\mathscr{B}_{\ell}$ whose support is entirely contained in $\Omega_{\ell}$.

Example 51. An example of the recursive definition of HB-splines is illustrated in Figure 4. We consider three nested knot sequences, with knots of multiplicity 4 at the two extrema of the intervals and single knots elsewhere, as in Figure 4(a). This allows us to construct the three sets of cubic Bsplines shown in Figure 4(c,e,g), whose dimensions are $n_{1}=10, n_{2}=17$ and $n_{3}=31$, respectively. The domain hierarchy is defined by the subsets $\Omega_{1}=\left[\xi_{4,1}, \xi_{11,1}\right], \Omega_{2}=\left[\xi_{8,2}, \xi_{16,2}\right]$ and $\Omega_{3}=$ $\left[\xi_{16,3}, \xi_{24,3}\right]$, and is shown in Figure 4(b). Obviously, $\mathscr{H}_{1}$ coincides with $\mathscr{B}_{1}$. Furthermore, $\mathscr{H}_{2}^{C}$

7 The HB-splines in Definition 50 were introduced by Kraft $[28,29]$ and further elaborated in [53]. However, the concept of hierarchical splines has a long history; for example, it was used in preconditioning [18, 54], adaptive modeling [19, 20] and adaptive finite elements [25, 30]. 




(a) knot sequences

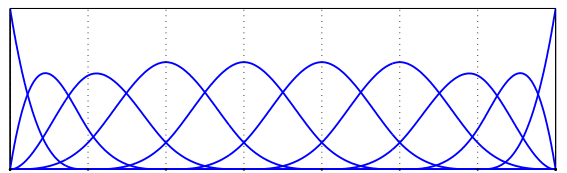

(c) $\mathscr{B}_{1}$

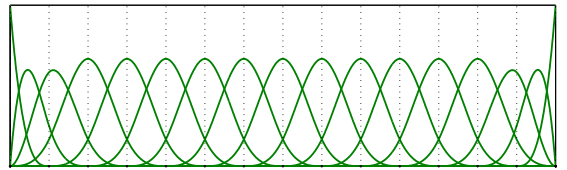

(e) $\mathscr{B}_{2}$

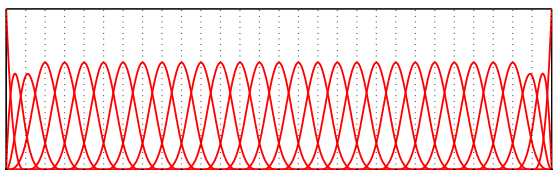

(g) $\mathscr{B}_{3}$



(b) domain hierarchy

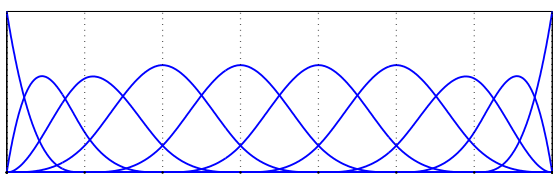

(d) $\mathscr{H}_{1}$

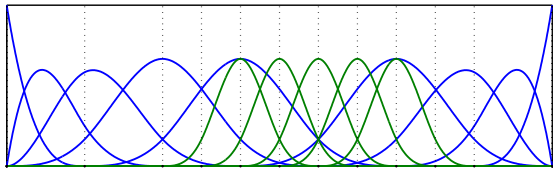

(f) $\mathscr{H}_{2}$

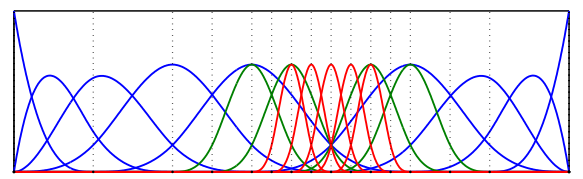

(h) $\mathscr{H}_{3}=\mathscr{H}_{\boldsymbol{\Omega}}$

Fig. 4 An example of cubic HB-splines where the domain hierarchy consists of three levels. The knot positions are visualized by vertical dotted lines in (c)-(h).

is obtained from $\mathscr{H}_{1}$ by removing $B_{6,1}$, and $\mathscr{H}_{2}^{F}=\left\{B_{8,2}, \ldots, B_{12,2}\right\}$. Hence, $\mathscr{H}_{2}=\mathscr{H}_{2}^{C} \cup \mathscr{H}_{2}^{F}$ consists of $9+5=14$ elements. Finally, $\mathscr{H}_{3}^{C}$ is obtained from $\mathscr{H}_{2}$ by removing $B_{10,2}$, and $\mathscr{H}_{3}^{F}=$ $\left\{B_{16,3}, \ldots, B_{20,3}\right\}$. Hence, $\mathscr{H}_{3}=\mathscr{H}_{3}^{C} \cup \mathscr{H}_{3}^{F}$ consists of $13+5=18$ elements. The sets $\mathscr{H}_{1}, \mathscr{H}_{2}$ and $\mathscr{H}_{3}$ are shown in Figure 4(d,f,h).

For each $\ell \in\{1, \ldots, L\}$, let $J_{\ell, \Omega}$ be the set of indices of the B-splines in $\mathscr{B}_{\ell}$ belonging to $\mathscr{H}_{\boldsymbol{\Omega}}$, i.e.,

$$
J_{\ell, \boldsymbol{\Omega}}:=\left\{j: B_{j, \ell} \in \mathscr{B}_{\ell} \cap \mathscr{H}_{\boldsymbol{\Omega}}\right\} .
$$

From Definition 50 it follows that

$$
J_{\ell, \boldsymbol{\Omega}}=\left\{j: B_{j, \ell} \in \mathscr{B}_{\ell}, \operatorname{supp}_{\boldsymbol{\Omega}}\left(B_{j, \ell}\right) \cap \Gamma_{\ell} \neq \emptyset, \operatorname{supp}_{\boldsymbol{\Omega}}\left(B_{j, \ell}\right) \subseteq \Omega_{\ell}\right\},
$$

where 


$$
\Gamma_{\ell}:=\Omega_{\ell} \backslash \Omega_{\ell+1} .
$$

Given this index set, we can reconstruct the set of HB-splines as

$$
\mathscr{H}_{\boldsymbol{\Omega}}=\left\{B_{j, \ell}, j \in J_{\ell, \boldsymbol{\Omega}}, \ell=1, \ldots, L\right\} .
$$

Since the set of HB-splines is a mixture of standard B-splines, we deduce immediately the following properties.

- Local Support. An HB-spline is locally supported on an interval that only depends on the level it was introduced in the hierarchical construction and not on the choice of subsets in the domain hierarchy.

- Nonnegativity. An HB-spline is nonnegative everywhere, and positive inside its support.

- Piecewise Structure. An HB-spline is a piecewise polynomial, whose degree and smoothness depends on the level it was introduced in the hierarchical construction and the spline space used on that level.

- Linear Independence. The HB-splines in $\mathscr{H}_{\boldsymbol{\Omega}}$ are linearly independent on $\Omega_{1}$.

Proof. We first note that if $J_{\ell, \Omega}$ is nonempty then $\Gamma_{\ell}$ has nonempty interior for any $\ell$; see (6.7) and (6.8). We must prove that if

$$
s(x)=\sum_{\ell=1}^{L} \sum_{j \in J_{\ell, \Omega}} c_{j, \ell} B_{j, \ell}(x)=0, \quad x \in \Omega_{1}
$$

then $c_{j, \ell}=0$ for all $j$ and $\ell$ in (6.10). We know from the local linear independence property that the B-splines $B_{j, 1}, j \in J_{1, \Omega}$ are linearly independent on $\Gamma_{1}$. Moreover, from (6.7) it follows that only those functions are nonzero on $\Gamma_{1}$. Hence, we conclude that $c_{j, 1}=0$ for $j \in J_{1, \Omega}$ in (6.10). We can repeat the same argument for the remaining terms in (6.10) going level by level in the hierarchy. Indeed, for $\ell=2, \ldots, L$, the B-splines $B_{j, \ell}, j \in J_{\ell, \Omega}$ are linearly independent on $\Gamma_{\ell}$, and only those functions are nonzero on $\Gamma_{\ell}$ except for functions already considered before at previous levels. This implies that $c_{j, \ell}=0$ for $j \in J_{\ell, \Omega}$ with $\ell=2, \ldots, L$.

The space spanned by the HB-splines in $\mathscr{H}_{\boldsymbol{\Omega}}$ is called the hierarchical spline space on $\boldsymbol{\Omega}$ and is denoted by

$$
\mathbb{S}_{\Omega}:=\left\{s: \Omega_{1} \rightarrow \mathbb{R}: s=\sum_{\ell=1}^{L} \sum_{j \in J_{\ell, \Omega}} c_{j, \ell} B_{j, \ell}, c_{j, \ell} \in \mathbb{R}\right\} .
$$

Such hierarchical space has some interesting properties.

- Dimension. By the linear independence of the HB-splines, the space $\mathbb{S}_{\boldsymbol{\Omega}}$ is a vector space of dimension $\sum_{\ell=1}^{L}\left|J_{\ell, \Omega}\right|$.

- Nestedness. Let the domain hierarchy $\tilde{\boldsymbol{\Omega}}$ be obtained from another domain hierarchy $\boldsymbol{\Omega}$ such that $\Omega_{1}=\tilde{\Omega}_{1}$ and $\Omega_{\ell} \subseteq \tilde{\Omega}_{\ell}$ for $\ell=2, \ldots, L$. Then, $\mathbb{S}_{\boldsymbol{\Omega}} \subseteq \mathbb{S}_{\tilde{\boldsymbol{\Omega}}}$. 
Proof. We first note that any B-spline $B_{j, \ell-1} \in \mathscr{B}_{\ell-1}$ whose support is entirely contained in $\Omega_{\ell}$ can be represented exactly in terms of B-splines $B_{i, \ell} \in \mathscr{B}_{\ell}$ whose support is also contained in $\Omega_{\ell}$. Consider the intermediate spaces $\mathscr{H}_{\ell}$ and $\tilde{\mathscr{H}}_{\ell}$ arising in Definition 50. From their construction it directly follows

$$
\operatorname{span}\left(\mathscr{H}_{\ell-1}\right) \subseteq \operatorname{span}\left(\mathscr{H}_{\ell}\right) \quad \text { and } \quad \operatorname{span}\left(\tilde{\mathscr{H}}_{\ell-1}\right) \subseteq \operatorname{span}\left(\tilde{\mathscr{H}}_{\ell}\right) .
$$

We now show that $\operatorname{span}\left(\mathscr{H}_{\ell}\right) \subseteq \operatorname{span}\left(\tilde{\mathscr{H}}_{\ell}\right)$ for all $\ell=1, \ldots, L$. This clearly holds for $\ell=1$ since $\Omega_{1}=\tilde{\Omega}_{1}$ and hence $\mathscr{H}_{1}=\tilde{\mathscr{H}}_{1}$. We proceed by induction on $\ell$, and assume that the statement is true for $\ell-1$. Then, we have

$$
\operatorname{span}\left(\mathscr{H}_{\ell}^{C}\right) \subseteq \operatorname{span}\left(\mathscr{H}_{\ell-1}\right) \subseteq \operatorname{span}\left(\tilde{\mathscr{H}}_{\ell-1}\right) \subseteq \operatorname{span}\left(\tilde{\mathscr{H}}_{\ell}\right)
$$

and

$$
\operatorname{span}\left(\mathscr{H}_{\ell}^{F}\right) \subseteq \operatorname{span}\left(\tilde{\mathscr{H}}_{\ell}^{F}\right) \subseteq \operatorname{span}\left(\tilde{\mathscr{H}}_{\ell}\right)
$$

This implies

$$
\operatorname{span}\left(\mathscr{H}_{\ell}\right)=\operatorname{span}\left(\mathscr{H}_{\ell}^{C}\right) \cup \operatorname{span}\left(\mathscr{H}_{\ell}^{F}\right) \subseteq \operatorname{span}\left(\tilde{\mathscr{H}}_{\ell}\right) .
$$

As a consequence, $\mathbb{S}_{\boldsymbol{\Omega}}=\operatorname{span}\left(\mathscr{H}_{L}\right) \subseteq \operatorname{span}\left(\tilde{\mathscr{H}}_{L}\right)=\mathbb{S}_{\tilde{\Omega}}$.

- Polynomial embedding. The space $\mathbb{S}_{\boldsymbol{\Omega}}$ contains (at least) all polynomials of degree less than or equal to $p_{1}$.

Proof. Let $g$ be a polynomial in $\mathbb{P}_{p_{1}}$. From Section 3.3 we know that $g$ belongs to the coarsest spline space $\mathbb{S}_{p_{1}, \boldsymbol{\xi}_{1}}$ in the sequence (6.1). Hence, taking into account (6.12), we conclude that $g \in \operatorname{span}\left(\mathscr{H}_{1}\right) \subseteq \operatorname{span}\left(\mathscr{H}_{L}\right)=\mathbb{S}_{\boldsymbol{\Omega}}$.

\subsection{Truncated Hierarchical B-Splines}

HB-splines do not satisfy the partition of unity property. In addition, the number of overlapping basis functions associated with different hierarchical levels easily increases. This motivates the construction of another basis for the hierarchical spline space. The construction is based on the following truncation mechanism [22].

Definition 52. Given $\ell \in\{2, \ldots, L\}$, let $s \in \mathbb{S}_{p_{\ell}, \xi_{\ell}}$ be represented in the B-spline basis $\mathscr{B}_{\ell}$, i.e.,

$$
s=\sum_{j=1}^{n_{\ell}} c_{j, \ell} B_{j, \ell} .
$$

The truncation of s at level $\ell$ is defined as the sum of the terms appearing in (6.13) related to the B-splines whose support is not a subset of $\Omega_{\ell}$, i.e.,

$$
\operatorname{trunc}_{\ell, \boldsymbol{\Omega}}(s):=\sum_{j: \sup _{\boldsymbol{\Omega}}\left(B_{j, \ell}\right) \nsubseteq \Omega_{\ell}} c_{j, \ell} B_{j, \ell} .
$$


By successively truncating the functions constructed in Definition 50, we obtain the truncated hierarchical B-splines (in short, THB-splines) ${ }^{8}$.

Definition 53. Given a domain hierarchy $\boldsymbol{\Omega}$, the corresponding set of $\mathbf{T H B}$-splines is denoted by $\mathscr{T}_{\Omega}$ and defined recursively as follows:

(i) $\mathscr{T}_{1}:=\left\{B_{j, 1} \in \mathscr{B}_{1}: \operatorname{supp}_{\Omega}\left(B_{j, 1}\right) \neq \emptyset\right\}$;

(ii) for $\ell=2, \ldots, L$ :

$$
\mathscr{T}_{\ell}:=\mathscr{T}_{\ell}^{C} \cup \mathscr{T}_{\ell}^{F}
$$

where

$$
\begin{aligned}
& \mathscr{T}_{\ell}^{C}:=\left\{\operatorname{trunc}_{\ell, \boldsymbol{\Omega}}\left(B_{j, k, \Omega_{\ell-1}}^{t}\right): B_{j, k, \Omega_{\ell-1}}^{t} \in \mathscr{T}_{\ell-1}, \operatorname{supp}_{\boldsymbol{\Omega}}\left(B_{j, k, \Omega_{\ell-1}}^{t}\right) \not \subseteq \Omega_{\ell}\right\}, \\
& \mathscr{T}_{\ell}^{F}:=\left\{B_{j, \ell} \in \mathscr{B}_{\ell}: \operatorname{supp}_{\boldsymbol{\Omega}}\left(B_{j, \ell}\right) \subseteq \Omega_{\ell}\right\} ;
\end{aligned}
$$

(iii) $\mathscr{T}_{\Omega}:=\mathscr{T}_{L}$.

To obtain the THB-splines, we apply a recursive procedure building a set $\mathscr{T}_{\ell}$ at level $\ell$. This set consists of two subsets, the coarse set $\mathscr{T}_{\ell}^{C}$ and the fine set $\mathscr{T}_{\ell}^{F}$. To construct the elements $B_{j, k, \Omega_{\ell}}^{t}$ of $\mathscr{T}_{\ell}^{C}$, we first express any function $B_{j, k, \Omega_{\ell-1}}^{t} \in \mathscr{T}_{\ell-1}$ with respect to the B-spline basis $\mathscr{B}_{\ell}$, and then we apply the truncation as in (6.14) with $s=B_{j, k, \Omega_{\ell-1}}^{t}$. The fine set $\mathscr{T}_{\ell}^{F}$ consists of all B-splines in $\mathscr{B}_{\ell}$ whose support is entirely contained in $\Omega_{\ell}$, exactly as in the HB-spline case; see Definition 50.

When comparing Definition 53 with Definition 50, we see that the number of THB-splines in the set $\mathscr{T}_{\boldsymbol{\Omega}}$ is equal to the number of HB-splines in the set $\mathscr{H}_{\boldsymbol{\Omega}}$. In the following, the THB-splines in $\mathscr{T}_{\Omega}$ are denoted by $B_{j, \ell, \Omega}^{T}$ for $j \in J_{\ell, \Omega}$ and $\ell=1, \ldots, L$.

Example 54. When unrolling the recursive definition of THB-splines for $L=3$, we get

$$
\begin{aligned}
& B_{j, 1, \boldsymbol{\Omega}}^{T}=\operatorname{trunc}_{3, \boldsymbol{\Omega}}\left(\operatorname{trunc}_{2, \boldsymbol{\Omega}}\left(B_{j, 1}\right)\right), \quad j \in J_{1, \boldsymbol{\Omega}}, \\
& B_{j, 2, \boldsymbol{\Omega}}^{T}=\operatorname{trunc}_{3, \boldsymbol{\Omega}}\left(B_{j, 2}\right), \quad j \in J_{2, \boldsymbol{\Omega}}, \\
& B_{j, 3, \boldsymbol{\Omega}}^{T}=B_{j, 3}, \quad j \in J_{3, \boldsymbol{\Omega}} .
\end{aligned}
$$

Example 55. Figure 5 illustrates the truncation mechanism applied to the set of HB-splines depicted in Figure 4 (Example 51). Obviously, $\mathscr{T}_{1}$ coincides with $\mathscr{H}_{1}$. Furthermore, $\mathscr{T}_{2}^{C}$ is obtained from $\mathscr{H}_{2}^{C}$ by applying the truncation mechanism to its elements; this only results in a modification of the elements $B_{4,1}, B_{5,1}, B_{7,1}$ and $B_{8,1}$. On the other hand, we have $\mathscr{T}_{2}^{F}=\mathscr{H}_{2}^{F}$. Finally, $\mathscr{T}_{3}^{C}$ is obtained from $\mathscr{H}_{3}^{C}$ by modifying $B_{4,1}, B_{5,1}, B_{7,1}, B_{8,1}$ (truncated at level 2) and $B_{8,2}, B_{9,2}, B_{11,2}$, $B_{12,2}$ (truncated at level 3), while $\mathscr{T}_{3}^{F}=\mathscr{H}_{3}^{F}$. It is clear that $\mathscr{T}_{\ell}=\mathscr{T}_{\ell}^{C} \cup \mathscr{T}_{\ell}^{F}$ and $\mathscr{H}_{\ell}=\mathscr{H}_{\ell}^{C} \cup \mathscr{H}_{\ell}^{F}$ have the same number of elements for $\ell=2,3$.

The next properties can be easily deduced from the definition of THB-splines.

- Relation to HB-Splines. Each THB-spline in $\mathscr{T}_{\Omega}$ is uniquely related to a single HB-spline in $\mathscr{H}_{\Omega}$ possibly by successive truncations, i.e.,

\footnotetext{
${ }^{8}$ The truncation approach was introduced in [22] for hierarchical tensor-product splines, but was already developed before in the context of hierarchical Powell-Sabin splines [50]. A generalization towards a broad class of hierarchical spaces can be found in [23].
} 


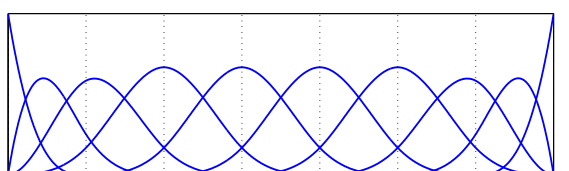

(a) $\mathscr{H}_{1}$



(c) $\mathscr{H}_{2}$

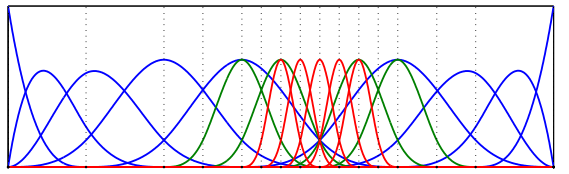

(e) $\mathscr{H}_{3}$

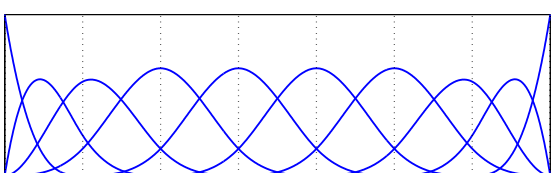

(b) $\mathscr{T}_{1}$

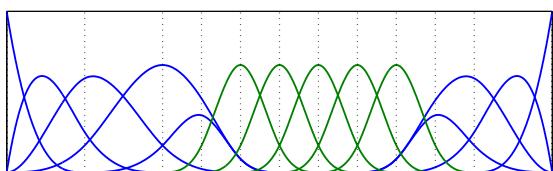

(d) $\mathscr{T}_{2}$

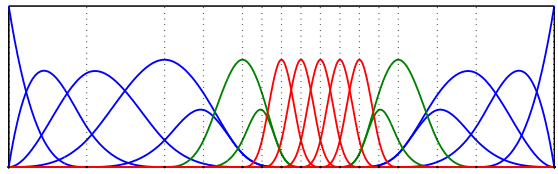

(f) $\mathscr{T}_{3}$

Fig. 5 HB-splines and THB-splines with respect to the same domain hierarchy as in Figure 4(b).

$$
B_{j, \ell, \Omega}^{T}=\operatorname{Trunc}_{\ell, \Omega}\left(B_{j, \ell}\right),
$$

where for any $s \in \mathbb{S}_{p_{\ell}, \xi_{\ell}}$ with $\ell=1, \ldots, L-1$,

$$
\operatorname{Trunc}_{\ell, \boldsymbol{\Omega}}(s):=\operatorname{trunc}_{L, \boldsymbol{\Omega}}\left(\operatorname{trunc}_{L-1, \boldsymbol{\Omega}}\left(\cdots\left(\operatorname{trunc}_{\ell+1, \Omega}(s)\right) \cdots\right)\right) \text {, }
$$

and for any $s \in \mathbb{S}_{p_{L}, \boldsymbol{\xi}_{L}}$,

$$
\operatorname{Trunc}_{L, \Omega}(s):=s .
$$

From (6.15) in combination with (6.13)-(6.14), it is clear that

$$
B_{j, \ell, \Omega}^{T}(x)=B_{j, \ell}(x), \quad x \in \Gamma_{\ell} .
$$

- Local Support. From (6.15) it follows that a THB-spline has the same or smaller support than its related HB-spline.

- Nonnegativity. A THB-spline is nonnegative everywhere.

Proof. Fix $1 \leq \ell_{1}<\ell_{2} \leq L$. Because of the nestedness of the spaces in (6.1), we can write the B-spline $B_{j, \ell_{1}} \in \mathscr{B}_{\ell_{1}}$ in terms of the B-splines in $\mathscr{B}_{\ell_{2}}$, i.e.,

$$
B_{j, \ell_{1}}=\sum_{i=1}^{n_{\ell_{2}}} c_{i, \ell_{2}}^{j, \ell_{1}} B_{i, \ell_{2}} .
$$


From Section 3.5 we know that the coefficients in (6.17) are all nonnegative in case $p_{\ell_{1}}=p_{\ell_{2}}$. This property holds in general, also when $p_{\ell_{1}}<p_{\ell_{2}}$, and we refer to [12] for its proof. Then, since each THB-spline $B_{j, \ell, \Omega}^{T}$ can be deduced from the B-spline $B_{j, \ell}$ possibly by successive truncations, see (6.15), it follows from (6.13)-(6.14) that $B_{j, \ell, \Omega}^{T}$ can be written as a linear combination of B-splines of the finest level $L$ with nonnegative coefficients. This implies that $B_{j, \ell, \Omega}^{T}$ is nonnegative.

- Linear Independence. The THB-splines in $\mathscr{T}_{\Omega}$ are linearly independent on $\Omega_{1}$.

Proof. We must prove that if

$$
s(x)=\sum_{\ell=1}^{L} \sum_{j \in J_{\ell, \Omega}} c_{j, \ell} B_{j, \ell}^{T}(x)=0, \quad x \in \Omega_{1},
$$

then $c_{j, \ell}=0$ for all $j$ and $\ell$ in (6.18). This can be shown using exactly the same line of arguments as in the case of HB-splines (see (6.10)), taking into account relation (6.16).

The next theorem shows that the THB-splines in $\mathscr{T}_{\boldsymbol{\Omega}}$ form an alternative basis for the hierarchical spline space $\mathbb{S}_{\boldsymbol{\Omega}}$.

Theorem 56 (Hierarchical Spline Space). The THB-splines in $\mathscr{T}_{\Omega}$ span the same space as the HB-splines in $\mathscr{H}_{\boldsymbol{\Omega}}$, i.e.,

$$
\mathbb{S}_{\boldsymbol{\Omega}}=\operatorname{span}\left(\mathscr{H}_{\boldsymbol{\Omega}}\right)=\operatorname{span}\left(\mathscr{T}_{\boldsymbol{\Omega}}\right) .
$$

Proof. Consider the intermediate spaces $\mathscr{H}_{\ell}$ and $\mathscr{T}_{\ell}$ in Definitions 50 and 53, respectively. From their construction it directly follows

$$
\operatorname{span}\left(\mathscr{H}_{\ell-1}\right) \subseteq \operatorname{span}\left(\mathscr{H}_{\ell}\right) \quad \text { and } \quad \operatorname{span}\left(\mathscr{T}_{\ell-1}\right) \subseteq \operatorname{span}\left(\mathscr{T}_{\ell}\right)
$$

We now show that $\operatorname{span}\left(\mathscr{H}_{\ell}\right)=\operatorname{span}\left(\mathscr{T}_{\ell}\right)$ for all $\ell=1, \ldots, L$. This clearly holds for $\ell=1$ since $\mathscr{H}_{1}=\mathscr{T}_{1}$. We proceed by induction on $\ell$, and assume that the statement is true for $\ell-1$. Then, we have

$$
\operatorname{span}\left(\mathscr{H}_{\ell}^{C}\right) \subseteq \operatorname{span}\left(\mathscr{H}_{\ell-1}\right)=\operatorname{span}\left(\mathscr{T}_{\ell-1}\right) \subseteq \operatorname{span}\left(\mathscr{T}_{\ell}\right),
$$

and

$$
\operatorname{span}\left(\mathscr{H}_{\ell}^{F}\right)=\operatorname{span}\left(\mathscr{T}_{\ell}^{F}\right) \subseteq \operatorname{span}\left(\mathscr{T}_{\ell}\right)
$$

This implies

$$
\operatorname{span}\left(\mathscr{H}_{\ell}\right)=\operatorname{span}\left(\mathscr{H}_{\ell}^{C}\right) \cup \operatorname{span}\left(\mathscr{H}_{\ell}^{F}\right) \subseteq \operatorname{span}\left(\mathscr{T}_{\ell}\right) .
$$

Finally, since both sets $\mathscr{H}_{\ell}$ and $\mathscr{T}_{\ell}$ have the same number of elements and these elements are all linearly independent, it follows that $\operatorname{span}\left(\mathscr{H}_{\ell}\right)=\operatorname{span}\left(\mathscr{T}_{\ell}\right)$. As a consequence, $\operatorname{span}\left(\mathscr{H}_{\boldsymbol{\Omega}}\right)=\operatorname{span}\left(\mathscr{H}_{L}\right)=\operatorname{span}\left(\mathscr{T}_{L}\right)=\operatorname{span}\left(\mathscr{T}_{\boldsymbol{\Omega}}\right)$. 
The correspondence in (6.15) between the THB-spline $B_{j, \ell, \Omega}^{T}$ and a particular B-spline $B_{j, \ell} \in \mathscr{B}_{\ell}$ has an important consequence, namely the so-called property of preservation of coefficients [23]. This means that the THB-spline representation preserves certain coefficients of functions represented with respect to one of the B-spline bases $\mathscr{B}^{\ell}$.

Theorem 57 (Preservation of Coefficients). Given $\ell \in\{1, \ldots, L\}$, let the restriction of $s \in \mathbb{S}_{\boldsymbol{\Omega}}$ to $\Gamma_{\ell}:=\Omega_{\ell} \backslash \Omega_{\ell+1}$ be represented in the bases $\mathscr{T}_{\boldsymbol{\Omega}}$ and $\mathscr{B}_{\ell}$, i.e.,

$$
s(x)=\sum_{k=1}^{L} \sum_{j \in J_{k, \boldsymbol{\Omega}}} c_{j, k}^{T} B_{j, k, \boldsymbol{\Omega}}^{T}(x)=\sum_{i=1}^{n_{\ell}} c_{i, \ell} B_{i, \ell}(x), \quad x \in \Gamma_{\ell} .
$$

Then,

$$
c_{i, \ell}^{T}=c_{i, \ell}, \quad i \in J_{\ell, \Omega} .
$$

Proof. Since $s \in \mathbb{S}_{\boldsymbol{\Omega}}$ and the spline spaces in (6.1) are nested, it is clear that the restriction of $s$ to $\Gamma_{\ell}$ can be expressed as a linear combination of the B-splines in $\mathscr{B}_{\ell}$ restricted to $\Gamma_{\ell}$ as in (6.20). Let us focus on the sum

$$
\sum_{j \in J_{k, \boldsymbol{\Omega}}} c_{j, k}^{T} B_{j, k, \boldsymbol{\Omega}}^{T}(x), \quad x \in \Gamma_{\ell},
$$

and consider three cases.

- If $k>\ell$, then the sum in (6.22) equals zero. Indeed, Definition 53 and (6.15) imply that

$$
\operatorname{supp}_{\boldsymbol{\Omega}}\left(B_{j, k, \boldsymbol{\Omega}}^{T}\right) \subseteq \operatorname{supp}_{\boldsymbol{\Omega}}\left(B_{j, k}\right) \subseteq \Omega_{k} \subseteq \Omega_{\ell+1},
$$

and consequently, we have $\operatorname{supp}_{\boldsymbol{\Omega}}\left(B_{j, k, \Omega}^{T}\right) \cap \Gamma_{\ell}=\emptyset$.

- We now consider the case $k=\ell$. From (6.16) it immediately follows

$$
\sum_{j \in J_{\ell, \boldsymbol{\Omega}}} c_{j, \ell}^{T} B_{j, \ell, \boldsymbol{\Omega}}^{T}(x)=\sum_{j \in J_{\ell, \boldsymbol{\Omega}}} c_{j, \ell}^{T} B_{j, \ell}(x), \quad x \in \Gamma_{\ell} .
$$

- Finally, let $k<\ell$. In view of the truncation mechanism, we prove that THBsplines introduced at levels less than $\ell$ in the hierarchy can only contribute in terms of B-splines $B_{i, \ell}$ with $i \notin J_{\ell, \Omega}$. To this end, let us rewrite the corresponding THB-splines $B_{j, k, \Omega}^{T}$ in terms of the B-spline basis $\mathscr{B}_{\ell}$,

$$
B_{j, k, \boldsymbol{\Omega}}^{T}(x)=\sum_{i=1}^{n_{\ell}} c_{i, \ell}^{j, k} B_{i, \ell}(x), \quad x \in \Gamma_{\ell} .
$$

Due to the definition of $B_{j, k, \Omega}^{T}$ and the truncation operation (6.14), we have

$$
c_{i, \ell}^{j, k}=0, \quad \text { if } i \in J_{\ell, \boldsymbol{\Omega}} .
$$

Hence, for $k<\ell$ we arrive at 


$$
\sum_{j \in J_{k, \boldsymbol{\Omega}}} c_{j, k}^{T} B_{j, k, \boldsymbol{\Omega}}^{T}(x)=\sum_{i \notin J_{\ell, \boldsymbol{\Omega}}}\left(\sum_{j \in J_{k, \boldsymbol{\Omega}}} c_{j, k}^{T} c_{i, \ell}^{j, k}\right) B_{i, \ell}(x), \quad x \in \Gamma_{\ell} .
$$

By combining the above three cases and taking into account the local linear independence of B-splines, we obtain the identity (6.20) where

$$
c_{i, \ell}= \begin{cases}c_{i, \ell}^{T}, & \text { if } i \in J_{\ell, \boldsymbol{\Omega}}, \\ \sum_{k=1}^{\ell-1} \sum_{j \in J_{k, \boldsymbol{\Omega}}} c_{j, k}^{T} c_{i, \ell}^{j, k}, & \text { otherwise }\end{cases}
$$

which in particular gives (6.21).

Thanks to Theorem 57, many interesting features of B-spline representations can be transferred to THB-spline representations.

- Representation of Polynomials. Any polynomial $g$ of degree $p_{1}$ can be represented as

$$
g(x)=\sum_{\ell=1}^{L} \sum_{j \in J_{\ell, \Omega}} \Lambda_{j, p_{\ell}, \xi_{\ell}}(g) B_{j, \ell, \Omega}^{T}(x), \quad x \in \Omega_{1},
$$

where $\Lambda_{j, p_{\ell}, \boldsymbol{\xi}_{\ell}}$ is defined in (3.23) with $p=p_{\ell}$ and $\boldsymbol{\xi}=\boldsymbol{\xi}_{\ell}$.

Proof. Using the nestedness of the spaces (6.1), it is clear that $g \in \mathbb{S}_{p_{\ell}, \boldsymbol{\xi}_{\ell}}$ for $\ell=1, \ldots, L$ and also that $g \in \mathbb{S}_{\Omega}$. Then, consider its representation with respect to $\mathscr{T}_{\Omega}$ and $\mathscr{B}_{\ell}$ for $\ell=1, \ldots, L$. Theorem 57 in combination with Proposition 21 concludes the proof.

- Partition of Unity. By (3.19) we have

$$
\sum_{\ell=1}^{L} \sum_{j \in J_{\ell, \boldsymbol{\Omega}}} B_{j, \ell, \boldsymbol{\Omega}}^{T}(x)=1, \quad x \in \Omega_{1},
$$

Since the THB-splines are nonnegative it follows that they form a nonnegative partition of unity on $\Omega_{1}$.

- Greville Points. By (3.20) we have

$$
x=\sum_{\ell=1}^{L} \sum_{j \in J_{\ell, \boldsymbol{\Omega}}} \xi_{j, p_{\ell}, \boldsymbol{\xi}_{\ell}}^{*} B_{j, \ell, \boldsymbol{\Omega}}^{T}(x), \quad x \in \Omega_{1},
$$

where $\xi_{j, p_{\ell}, \boldsymbol{\xi}_{\ell}}^{*}$ are the Greville points defined in (3.21) with $p=p_{\ell}$ and $\boldsymbol{\xi}=\boldsymbol{\xi}_{\ell}$. Note that the Greville points are not necessarily distinct here.

- Strong Stability. The THB-spline basis is strongly stable with respect to the supremum norm, under mild assumptions on the underlying knot sequences required in the hierarchical construction. We refer to [23] for a proof based on the property of preservation of coefficients. Strong stability in the hierarchical context means that the constants to be considered in the stability relation (3.34) of the basis do not depend on the number of hierarchical levels. 
Example 58. The polynomial $g(x)=a x^{2}+b x+c$ can be represented in terms of quadratic THBsplines:

$$
a x^{2}+b x+c=\sum_{\ell=1}^{L} \sum_{j \in J_{\ell, \Omega}} c_{j, \ell} B_{j, \ell, \Omega}^{T}(x)
$$

From Theorem 57 and Example 22 we obtain that

$$
c_{j, \ell}=\Lambda_{j, 2, \xi_{\ell}}(g)=a \xi_{j+1, \ell} \xi_{j+2, \ell}+b \frac{\xi_{j+1, \ell}+\xi_{j+2, \ell}}{2}+c .
$$

\subsection{Quasi-Interpolation in Hierarchical Spaces}

The above properties of THB-splines can be exploited to develop a general and very simple procedure for the construction of quasi-interpolants in hierarchical spline spaces [51].

Definition 59. Given for each spline space in (6.1) a quasi-interpolant in B-spline form, i.e.,

$$
\mathscr{Q}_{\ell} f(x):=\sum_{j=1}^{n_{\ell}} \lambda_{j, \ell}(f) B_{j, \ell}(x), \quad x \in \Omega_{1}, \quad \ell=1, \ldots, L,
$$

the corresponding hierarchical quasi-interpolant in $\mathbb{S}_{\boldsymbol{\Omega}}$ is defined by

$$
\mathscr{Q}_{\boldsymbol{\Omega}} f(x):=\sum_{\ell=1}^{L} \sum_{j \in J_{\ell, \Omega}} \lambda_{j, \ell}(f) B_{j, \ell, \boldsymbol{\Omega}}^{T}(x), \quad x \in \Omega_{1} .
$$

According to Definition 59 , in order to construct a quasi-interpolant in $\mathbb{S}_{\boldsymbol{\Omega}}$, it suffices to consider first a quasi-interpolant in each space associated with a particular level in the hierarchy. Then, the coefficients of the proposed hierarchical quasiinterpolant are nothing else than a proper subset of the coefficients of the one-level quasi-interpolants.

We now show how to build hierarchical quasi-interpolants reproducing polynomials of a certain degree $p \leq p_{1}$. As described in Section 5.2, this is a crucial property to ensure good approximation properties.

Theorem 60 (Polynomial Reproduction). Let $\mathscr{Q}_{\ell}$ be a given sequence of quasiinterpolants as in (6.26), let $\mathscr{Q}_{\Omega}$ be the corresponding hierarchical quasi-interpolant as in (6.27), and let $p \leq p_{1}$. If

$$
\mathscr{Q}_{\ell} g=g, \quad \forall g \in \mathbb{P}_{p}, \quad \ell=1, \ldots, L,
$$

then

$$
\mathscr{Q}_{\boldsymbol{\Omega}} g=g, \quad \forall g \in \mathbb{P}_{p} .
$$

Proof. Since the spaces in (6.1) are nested, we have $p_{\ell} \geq p$ for all $\ell$. Let $g \in \mathbb{P}_{p} \subseteq$ $\mathbb{P}_{p_{\ell}} \subseteq \mathbb{S}_{p_{\ell}, \boldsymbol{\xi}_{\ell}}$. Then, this polynomial can be uniquely represented as a linear combi- 
nation of the B-splines in $\mathscr{B}_{\ell}$,

$$
g(x)=\sum_{j=1}^{n_{\ell}} c_{j, \ell} B_{j, \ell}(x),
$$

and since $\mathscr{Q}_{\ell} g=g$ we have $\lambda_{j, \ell}(g)=c_{j, \ell}$. On the other hand, $g \in \mathbb{S}_{\Omega}$, so

$$
g(x)=\sum_{\ell=1}^{L} \sum_{j \in J_{\ell, \boldsymbol{\Omega}}} c_{j, \ell}^{T} B_{j, \ell, \boldsymbol{\Omega}}^{T}(x) .
$$

From Theorem 57 it follows

$$
c_{j, \ell}^{T}=c_{j, \ell}=\lambda_{j, \ell}(g), \quad j \in J_{\ell, \Omega}, \quad \ell=1, \ldots, L,
$$

implying that $\mathscr{Q}_{\Omega} g=g$.

In the next theorem we present a sufficient condition for constructing quasiinterpolants that are projectors onto $\mathbb{S}_{\boldsymbol{\Omega}}$.

Theorem 61 (Spline Reproduction). Let $\mathscr{Q}_{\ell}$ be a given sequence of quasi-interpolants as in (6.26), and let $\mathscr{Q}_{\boldsymbol{\Omega}}$ be the corresponding hierarchical quasi-interpolant as in (6.27). Assume

$$
\mathscr{Q}_{\ell} s=s, \quad \forall s \in \mathbb{S}_{p_{\ell}, \boldsymbol{\xi}_{\ell}}, \quad \ell=1, \ldots, L,
$$

and each $\lambda_{j, \ell}$ used in (6.27) is supported on $\Gamma_{\ell}:=\Omega_{\ell} \backslash \Omega_{\ell+1}$. Then,

$$
\mathscr{Q}_{\boldsymbol{\Omega}} s=s, \quad \forall s \in \mathbb{S}_{\boldsymbol{\Omega}} .
$$

Proof. Due to the linearity of the quasi-interpolant, it suffices to prove that

$$
\lambda_{j, \ell}\left(B_{i, k, \boldsymbol{\Omega}}^{T}\right)=\delta_{i, j} \delta_{k, \ell}, \quad i \in J_{k, \boldsymbol{\Omega}}, \quad j \in J_{\ell, \boldsymbol{\Omega}}, \quad k, \ell=1, \ldots, L,
$$

where $\delta_{r, s}$ stands for the classical Kronecker delta. Let $j$ and $\ell$ be fixed. To prove (6.29) we consider three cases.

- If $k>\ell$, then $B_{i, k, \boldsymbol{\Omega}}^{T}(x)=0$ for $x \in \Gamma_{\ell}$; see Definition 53. Since $\lambda_{j, \ell}$ is only supported on $\Gamma_{\ell}$, it follows from Definition 33 that $\lambda_{j, \ell}\left(B_{i, k, \Omega}^{T}\right)=0$.

- We now consider the case $k=\ell$. Since $\mathscr{Q}_{\ell}$ is a projector onto $\mathbb{S}_{\ell}$, we have that $\lambda_{j, \ell}\left(B_{i, \ell}\right)=\delta_{i, j}$. From (6.16) and the support restriction of $\lambda_{j, \ell}$, we obtain

$$
\lambda_{j, \ell}\left(B_{i, \ell, \boldsymbol{\Omega}}^{T}\right)=\delta_{i, j}, \quad i, j \in J_{\ell, \Omega} .
$$

- Finally, let $k<\ell$. Any $B_{i, k, \Omega}^{T}$ restricted to $\Gamma_{\ell}$ can then be expressed as a linear combination of the B-splines in $\mathscr{B}_{\ell}$ restricted to $\Gamma_{\ell}$, i.e.,

$$
B_{i, k, \boldsymbol{\Omega}}^{T}(x)=\sum_{r=1}^{n_{\ell}} c_{r, \ell}^{i, k} B_{r, \ell}(x), \quad x \in \Gamma_{\ell},
$$


where

$$
c_{r, \ell}^{i, k}=0, \quad \text { if } r \in J_{\ell, \Omega},
$$

as explained in the third case of the proof of Theorem 57. Thus, by the support restriction of $\lambda_{j, \ell}$, we have for $j \in J_{\ell, \Omega}$,

$$
\lambda_{j, \ell}\left(B_{i, k, \boldsymbol{\Omega}}^{T}\right)=\sum_{r=1}^{n_{\ell}} c_{r, \ell}^{i, k} \lambda_{j, \ell}\left(B_{r, \ell}\right)=\sum_{r=1}^{n_{\ell}} c_{r, \ell}^{i, k} \delta_{j, r}=c_{j, \ell}^{i, k}=0 .
$$

The above three cases complete the proof.

Some remarks are in order here.

- Constraints on (6.26). The sequence of quasi-interpolants (6.26) considered in Theorem 61 needs to satisfy constraints more restrictive than those in Theorem 60: For each level $\ell, \mathscr{Q}_{\ell}$ must be a projector onto $\mathbb{S}_{p_{\ell}, \boldsymbol{\xi}_{\ell}}$ and each $\lambda_{j, \ell}$, $j \in J_{\ell, \Omega}$, must be supported on $\Gamma_{\ell}$. The former constraint connects the sequence of quasi-interpolants $\mathscr{Q}_{1}, \ldots, \mathscr{Q}_{L}$ with the sequence of spaces $\mathbb{S}_{p_{1}, \boldsymbol{\xi}_{1}}, \ldots, \mathbb{S}_{p_{L}, \boldsymbol{\xi}_{L}}$ and has a similar counterpart in Theorem 60. The latter constraint links the same sequence of quasi-interpolants with the domain hierarchy $\boldsymbol{\Omega}$. Nevertheless, once a sequence of quasi-interpolants as in (6.26) satisfying the hypotheses of Theorem 61 is available, the construction of a hierarchical quasi-interpolant that is a projector onto $\mathbb{S}_{\boldsymbol{\Omega}}$ does not require additional efforts compared to a hierarchical quasi-interpolant that just reproduces polynomials.

- Dual Basis. Let $\left\{\lambda_{j, \ell}\right\}$ be a set of linear functionals as in (6.27) that provide a projector onto $\mathbb{S}_{\Omega}$. Then, because of (6.29), it is a dual basis for the THB-spline basis $\mathscr{T}_{\Omega}$.

- Approximation Power. Polynomial reproduction is one of the key ingredients to show the approximation power of spline quasi-interpolants; see Section 5.2. Boundedness of a hierarchical quasi-interpolation operator and optimal approximation accuracy can be achieved on domain hierarchies that are nicely graded (i.e., the boundaries of the different $\Omega_{\ell}$ are sufficiently separated). Local error estimates for hierarchical quasi-interpolants of the form (6.27) can be found in [51] with respect to the $L_{\infty}$-norm, and in [49] with respect to the general $L_{q}$-norm, $1 \leq q \leq \infty$.

Example 62. Let $p_{\ell}=2$, and let $\xi_{\ell}$ be a 3-open knot sequence with at most double knots in the interior for each $\ell=1, \ldots, L$. Then, we can choose the quasi-interpolants in (6.26) as in Example 41. This leads to the hierarchical quasi-interpolant

$$
\mathscr{Q}_{\boldsymbol{\Omega}} f(x)=\sum_{\ell=1}^{L} \sum_{j \in J_{\ell, \boldsymbol{\Omega}}} \lambda_{j, \ell}(f) B_{j, \ell, \boldsymbol{\Omega}}^{T}(x), \quad x \in \Omega_{1},
$$

where

$$
\lambda_{j, \ell}(f)=-\frac{1}{2} f\left(\xi_{j+1, \ell}\right)+2 f\left(\xi_{j, 2, \boldsymbol{\xi}_{\ell}}^{*}\right)-\frac{1}{2} f\left(\xi_{j+2, \ell}\right) .
$$

From Example 41 and Theorem 60 we deduce that this hierarchical quasi-interpolant reproduces the polynomial space $\mathbb{P}_{2}$. If $\left[\xi_{j+1, \ell}, \xi_{j+2, \ell}\right] \subseteq \Gamma_{\ell}$ for each $j \in J_{\ell, \Omega}$, then it actually reproduces the entire hierarchical spline space $\mathbb{S}_{\Omega}$, according to Theorem 61 . 
Example 63. Consider the quasi-interpolant constructed in Section 5.3.1 for each space $\mathbb{S}_{p_{\ell}, \boldsymbol{\xi}_{\ell}}$ of level $\ell=1, \ldots, L$. This leads to the hierarchical quasi-interpolant

$$
\mathscr{Q}_{\boldsymbol{\Omega}} f(x)=\sum_{\ell=1}^{L} \sum_{j \in J_{\ell, \Omega}} \mathscr{L}_{j, p_{\ell}, \boldsymbol{\xi}_{\ell}}(f) B_{j, \ell, \boldsymbol{\Omega}}^{T}(x), \quad x \in \Omega_{1},
$$

where $\mathscr{L}_{j, p_{\ell}, \boldsymbol{\xi}_{\ell}}$ is defined in (5.33) with $p=p_{\ell}$ and $\boldsymbol{\xi}=\boldsymbol{\xi}_{\ell}$; it is supported on a single knot interval $\left[\xi_{m_{j, p_{\ell}, \ell},}, \xi_{m_{j, p_{\ell}}+1, \ell}\right]$. From Lemma 43 and Theorem 60 we deduce that this hierarchical quasiinterpolant reproduces the polynomial space $\mathbb{P}_{p_{1}}$. Theorem 61 says that if $\left[\xi_{m_{j, p_{\ell}, \ell},}, \xi_{m_{j, p_{\ell}}+1, \ell}\right] \subseteq \Gamma_{\ell}$ for each $j \in J_{\ell, \Omega}$, then the hierarchical quasi-interpolant reproduces the entire hierarchical spline space $\mathbb{S}_{\boldsymbol{\Omega}}$.

The hierarchical quasi-interpolant in Definition 59 can be interpreted as a telescopic approximant, where for each level an approximant of the residual is added ${ }^{9}$. To show this, we define the following set of indices

$$
K_{\ell, \Omega}:=\left\{j: B_{j, \ell} \in \mathscr{B}_{\ell}, \operatorname{supp}_{\boldsymbol{\Omega}}\left(B_{j, \ell}\right) \subseteq \Omega_{\ell}\right\}
$$

Referring to (6.7), it is easy to see that $J_{\ell, \Omega} \subseteq K_{\ell, \Omega}$, and moreover $J_{L, \boldsymbol{\Omega}}=K_{L, \boldsymbol{\Omega}}$.

Theorem 64 (Telescopic Representation). Let $\mathscr{Q}_{\ell}$ be a given sequence of quasiinterpolants as in (6.26), and let $\mathscr{Q}_{\boldsymbol{\Omega}}$ be the corresponding hierarchical quasiinterpolant as in (6.27). Assume

$$
\mathscr{Q}_{\ell} s=s, \quad \forall s \in \mathbb{S}_{p_{\ell}, \boldsymbol{\xi}_{\ell}}, \quad \ell=1, \ldots, L
$$

then

$$
\mathscr{Q}_{\Omega} f=\sum_{\ell=1}^{L} f^{(\ell)}
$$

where

$$
\begin{aligned}
f^{(1)} & :=\sum_{j \in K_{1, \Omega}} \lambda_{j, 1}(f) B_{j, 1}, \\
f^{(\ell)} & :=\sum_{j \in K_{\ell, \Omega}} \lambda_{j, \ell}\left(f-f^{(1)}-\ldots-f^{(\ell-1)}\right) B_{j, \ell}, \quad \ell=2, \ldots, L .
\end{aligned}
$$

Proof. Each quasi-interpolant $\mathscr{Q}_{\ell}, \ell=1, \ldots, L$, is assumed to be a projector onto the space $\mathbb{S}_{p_{\ell}, \boldsymbol{\xi}_{\ell}}$, and because of the nestedness of the spaces $\mathbb{S}_{p_{\ell}, \boldsymbol{\xi}_{\ell}} \subset \mathbb{S}_{p_{\ell+1}, \boldsymbol{\xi}_{\ell+1}}$, we know that every basis function $B_{j, \ell}$ can be represented as

$$
B_{j, \ell}=\sum_{k=1}^{n_{\ell+1}} \lambda_{k, \ell+1}\left(B_{j, \ell}\right) B_{k, \ell+1}
$$

where $\lambda_{k, \ell+1}\left(B_{j, \ell}\right)=0$ if the support of $B_{k, \ell+1}$ is not contained in the support of $B_{j, \ell}$. By exploiting the definition of the truncated basis (6.15) and (6.33), we obtain

${ }^{9}$ The telescopic expression for the hierarchical quasi-interpolant was presented in [51]. A special telescopic approximation in the hierarchical setting was already considered in [29]. 


$$
\begin{aligned}
f^{(1)} & =\sum_{j \in K_{1, \Omega}} \lambda_{j, 1}(f) B_{j, 1} \\
& =\sum_{j \in J_{1, \Omega}} \lambda_{j, 1}(f) B_{j, 1, \Omega}^{T}+\sum_{j \in K_{1, \Omega}} \lambda_{j, 1}(f)\left(\sum_{k \in K_{2, \Omega}} \lambda_{k, 2}\left(B_{j, 1}\right) B_{k, 2}\right) .
\end{aligned}
$$

Moreover,

$$
\begin{aligned}
f^{(2)} & =\sum_{j \in K_{2, \Omega}} \lambda_{j, 2}(f) B_{j, 2}-\sum_{k \in K_{2, \Omega}} \lambda_{k, 2}\left(f^{(1)}\right) B_{k, 2} \\
& =\sum_{j \in K_{2, \Omega}} \lambda_{j, 2}(f) B_{j, 2}-\sum_{k \in K_{2, \Omega}}\left(\sum_{j \in K_{1, \Omega}} \lambda_{j, 1}(f) \lambda_{k, 2}\left(B_{j, 1}\right)\right) B_{k, 2} .
\end{aligned}
$$

Hence,

$$
f^{(1)}+f^{(2)}=\sum_{j \in J_{1, \Omega}} \lambda_{j, 1}(f) B_{j, 1, \Omega}^{T}+\sum_{j \in K_{2, \Omega}} \lambda_{j, 2}(f) B_{j, 2} .
$$

We now remark that from the truncation definition (6.14)-(6.15) it follows that $\lambda_{k, 3}\left(B_{j, 1, \boldsymbol{\Omega}}^{T}\right)=0$ for any $k \in K_{3, \boldsymbol{\Omega}}$ and $j \in J_{1, \boldsymbol{\Omega}}$, and so

$$
\sum_{k \in K_{3, \Omega}} \lambda_{k, 3}\left(B_{j, 1, \Omega}^{T}\right) B_{k, 3}=0, \quad \forall j \in J_{1, \Omega} .
$$

By using similar arguments as before, we can write (6.34) as

$$
\begin{aligned}
& f^{(1)}+f^{(2)}= \\
& \quad \sum_{j \in J_{1, \Omega}} \lambda_{j, 1}(f) B_{j, 1, \Omega}^{T}+\sum_{j \in J_{2, \Omega}} \lambda_{j, 2}(f) B_{j, 2, \boldsymbol{\Omega}}^{T}+\sum_{j \in K_{2, \Omega}} \lambda_{j, 2}(f)\left(\sum_{k \in K_{3, \Omega}} \lambda_{k, 3}\left(B_{j, 2}\right) B_{k, 3}\right),
\end{aligned}
$$

and by means of (6.34) and (6.35) we obtain

$$
\begin{aligned}
f^{(3)} & =\sum_{j \in K_{3, \Omega}} \lambda_{j, 3}(f) B_{j, 3}-\sum_{k \in K_{3, \Omega}} \lambda_{k, 3}\left(f^{(1)}+f^{(2)}\right) B_{k, 3} \\
& =\sum_{j \in K_{3, \Omega}} \lambda_{j, 3}(f) B_{j, 3}-\sum_{k \in K_{3, \Omega}}\left(\sum_{j \in K_{2, \Omega}} \lambda_{j, 2}(f) \lambda_{k, 3}\left(B_{j, 2}\right)\right) B_{k, 3},
\end{aligned}
$$

resulting in

$$
f^{(1)}+f^{(2)}+f^{(3)}=\sum_{j \in J_{1, \Omega}} \lambda_{j, 1}(f) B_{j, 1, \Omega}^{T}+\sum_{j \in J_{2, \Omega}} \lambda_{j, 2}(f) B_{j, 2, \boldsymbol{\Omega}}^{T}+\sum_{j \in K_{3, \Omega}} \lambda_{j, 3}(f) B_{j, 3} .
$$

By iterating over all levels in the hierarchy and repeating the same arguments, we get the relation $(6.31)$.

The telescopic representation in Theorem 64 directly leads to the representation of the hierarchical quasi-interpolant in terms of the HB-spline basis, instead of in terms of the THB-spline basis (see Definition 59), under assumption (6.30). Indeed, 
as observed in [51], thanks to property (6.33), one can simply replace the index sets $K_{\ell, \Omega}$ by $J_{\ell, \Omega}$ in (6.32) and the relation (6.31) still remains true. This implies that (6.31) can be rewritten as

$$
\mathscr{Q}_{\boldsymbol{\Omega}} f=\sum_{\ell=1}^{L} \sum_{j \in J_{\ell, \boldsymbol{\Omega}}} \lambda_{j, \ell}\left(f-\mathscr{Q}_{\ell-1, \boldsymbol{\Omega}} f\right) B_{j, \ell},
$$

where

$$
\mathscr{Q}_{0, \Omega} f:=0, \quad \mathscr{Q}_{r, \Omega} f:=\sum_{k=1}^{r} \sum_{j \in J_{k, \Omega}} \lambda_{j, k}\left(f-\mathscr{Q}_{k-1, \Omega} f\right) B_{j, k}, \quad r \geq 1 .
$$

\section{Tensor-Product Structures and Adaptive Extensions}

The most easy way to extend many of the previous results to the multivariate setting is to consider a tensor-product structure. For the sake of simplicity, we briefly focus here on the bivariate setting. The extension to higher dimensions is straightforward; it only requires a more involved indexing notation.

\subsection{Tensor-Product B-Splines}

Given two knot sequences

$$
\boldsymbol{\xi}_{k}:=\left\{\xi_{1, k} \leq \xi_{2, k} \leq \cdots \leq \xi_{n_{k}+p_{k}+1, k}\right\}, \quad k=1,2,
$$

we define the basic rectangle as

$$
R:=\left[\xi_{p_{1}+1,1}, \xi_{n_{1}+1,1}\right] \times\left[\xi_{p_{2}+1,2}, \xi_{n_{2}+1,2}\right] .
$$

Then, we can simply construct the tensor-product B-splines as the product of univariate $\mathrm{B}$-splines in each variable, i.e.,

$$
B_{j_{1}, j_{2}, p_{1}, p_{2}, \xi_{1}, \xi_{2}}\left(x_{1}, x_{2}\right):=B_{j_{1}, p_{1}, \xi_{1}}\left(x_{1}\right) B_{j_{2}, p_{2}, \xi_{2}}\left(x_{2}\right),
$$

for $j_{k}=1, \ldots, n_{k}$ and $k=1,2$.

Example 65. Figure 6 shows a schematic representation of a tensor-product B-spline basis of bidegree $\left(p_{1}, p_{2}\right)=(3,3)$. A $\left(p_{k}+1\right)$-open knot sequence is chosen in each direction $x_{k}$, where the interior knots are all simple, and the corresponding univariate B-splines are depicted. Then, the set of tensor-product B-splines is obtained by computing the tensor product of the sets of univariate B-splines in each direction. Contour plots of some bicubic tensor-product B-splines are depicted in Figure 7. 

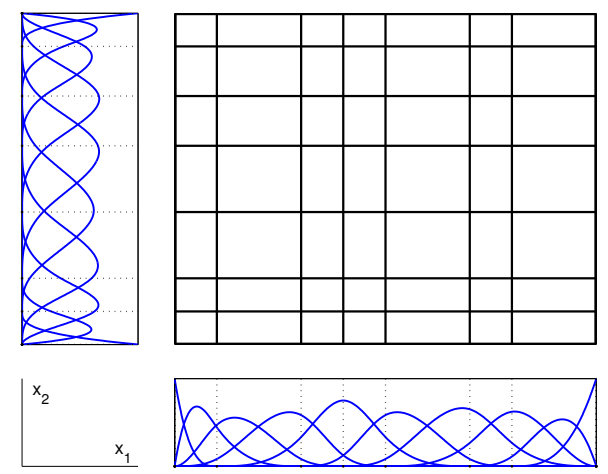

Fig. 6 Schematic representation of the (bivariate) tensor-product B-spline basis of bidegree $\left(p_{1}, p_{2}\right)=(3,3)$ using a 4-open knot sequence in each direction. The knot lines are visualized by solid lines in the rectangular domain (this is the basic rectangle), and the sets of univariate B-splines are depicted for both directions.

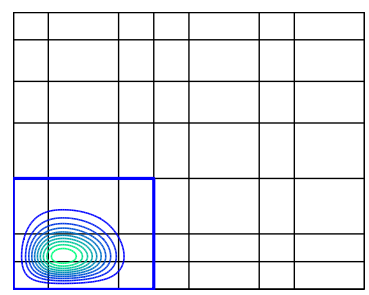

(a) $\left(j_{1}, j_{2}\right)=(3,3)$

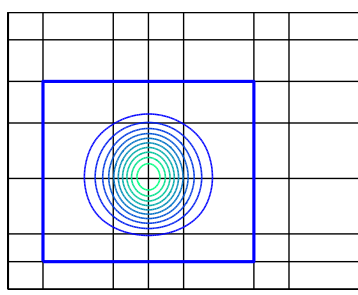

(b) $\left(j_{1}, j_{2}\right)=(5,5)$

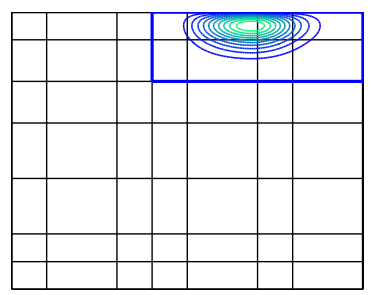

(c) $\left(j_{1}, j_{2}\right)=(7,9)$

Fig. 7 Contour plots of some bicubic tensor-product B-splines $B_{j_{1}, j_{2}, 3,3, \boldsymbol{\xi}_{1}, \boldsymbol{\xi}_{2}}$ defined on the tensorproduct mesh given in Figure 6 . The bounding box of the support of each $\mathrm{B}$-spline is visualized by solid blue lines.

It is clear that tensor-product B-splines inherit all the nice features of univariate B-splines discussed in Sections 2 and 3. In particular, they enjoy the following properties.

- Local Support. A tensor-product B-spline is locally supported on the rectangle given by the extreme knots used in the definition of its univariate B-splines in each direction, i.e.,

$$
B_{j_{1}, j_{2}, p_{1}, p_{2}, \boldsymbol{\xi}_{1}, \boldsymbol{\xi}_{2}}\left(x_{1}, x_{2}\right)=0, \quad\left(x_{1}, x_{2}\right) \notin S,
$$

where

$$
S:=\left[\xi_{j_{1}, 1}, \xi_{j_{1}+p_{1}+1,1}\right) \times\left[\xi_{j_{2}, 2}, \xi_{j_{2}+p_{2}+1,2}\right) .
$$

- Nonnegativity. A tensor-product B-spline is nonnegative everywhere, and positive inside its support, i.e., 


$$
B_{j_{1}, j_{2}, p_{1}, p_{2}, \boldsymbol{\xi}_{1}, \boldsymbol{\xi}_{2}}\left(x_{1}, x_{2}\right) \geq 0, \quad x_{1}, x_{2} \in \mathbb{R}
$$

and

$$
B_{j_{1}, j_{2}, p_{1}, p_{2}, \boldsymbol{\xi}_{1}, \boldsymbol{\xi}_{2}}\left(x_{1}, x_{2}\right)>0, \quad\left(x_{1}, x_{2}\right) \in \stackrel{\circ}{S}
$$

where

$$
\stackrel{\circ}{S}:=\left(\xi_{j_{1}, 1}, \xi_{j_{1}+p_{1}+1,1}\right) \times\left(\xi_{j_{2}, 2}, \xi_{j_{2}+p_{2}+1,2}\right) .
$$

- Piecewise Structure. A tensor-product B-spline has a piecewise tensor-product polynomial structure, i.e.,

$$
B_{j_{1}, j_{2}, p_{1}, p_{2}, \boldsymbol{\xi}_{1}, \boldsymbol{\xi}_{2}} \in \mathbb{P}_{p_{1}}\left(\left[\boldsymbol{\xi}_{m_{1}, 1}, \boldsymbol{\xi}_{m_{1}+1,1}\right)\right) \otimes \mathbb{P}_{p_{2}}\left(\left[\xi_{m_{2}, 2}, \xi_{m_{2}+1,2}\right)\right) .
$$

- Smoothness. If $\xi$ is a knot of $B_{j_{k}, p_{k}, \xi_{k}}$ of multiplicity $\mu \leq p_{k}+1$ then $B_{j_{1}, j_{2}, p_{1}, p_{2}, \boldsymbol{\xi}_{1}, \boldsymbol{\xi}_{2}}$ belongs to the class $C^{p_{k}-\mu}$ across the line $x_{k}=\xi$ for $k=1,2$.

- Linear Independence. If each $\boldsymbol{\xi}_{k}$ is $\left(p_{k}+1\right)$-basic for $k=1,2$, then the tensorproduct B-splines $\left\{B_{j_{1}, j_{2}, p_{1}, p_{2}, \boldsymbol{\xi}_{1}, \boldsymbol{\xi}_{2}}: j_{k}=1, \ldots, n_{k}, k=1,2\right\}$ are (locally) linearly independent on $R$.

- Partition of Unity. We have

$$
\sum_{j_{1}=1}^{n_{1}} \sum_{j_{2}=1}^{n_{2}} B_{j_{1}, j_{2}, p_{1}, p_{2}, \boldsymbol{\xi}_{1}, \boldsymbol{\xi}_{2}}\left(x_{1}, x_{2}\right)=1, \quad\left(x_{1}, x_{2}\right) \in R .
$$

Since the tensor-product B-splines are nonnegative it follows that they form a nonnegative partition of unity on $R$.

- Greville Points. We have

$$
x_{1}^{\ell_{1}} x_{2}^{\ell_{2}}=\sum_{j_{1}=1}^{n_{1}} \sum_{j_{2}=1}^{n_{2}}\left(\xi_{j_{1}, p_{1}, \xi_{1}}^{*}\right)^{\ell_{1}}\left(\xi_{j_{2}, p_{2}, \boldsymbol{\xi}_{2}}^{*}\right)^{\ell_{2}} B_{j_{1}, j_{2}, p_{1}, p_{2}, \xi_{1}, \xi_{2}}\left(x_{1}, x_{2}\right), \quad\left(x_{1}, x_{2}\right) \in R,
$$

for $\ell_{k} \in\{0,1\}$, and $\xi_{j_{k}, p_{k}, \boldsymbol{\xi}_{k}}^{*}$ is the Greville point defined in (3.21) for the knot sequence $\boldsymbol{\xi}_{k}$.

A tensor-product spline function is defined as

$$
s\left(x_{1}, x_{2}\right)=\sum_{j_{1}=1}^{n_{1}} \sum_{j_{2}=1}^{n_{2}} c_{j_{1}, j_{2}} B_{j_{1}, j_{2}, p_{1}, p_{2}, \boldsymbol{\xi}_{1}, \boldsymbol{\xi}_{2}}\left(x_{1}, x_{2}\right), \quad c_{j_{1}, j_{2}} \in \mathbb{R} .
$$

Since the tensor-product B-splines are linearly independent, the space of spline functions has dimension $n_{1} n_{2}$.

A main advantage of the representation in (7.8) is that its evaluation can be reduced to a sequence of evaluations of univariate spline functions:

$$
s\left(x_{1}, x_{2}\right)=\sum_{j_{1}=1}^{n_{1}} d_{j_{1}, x_{2}} B_{j_{1}, p_{1}, \boldsymbol{\xi}_{1}}\left(x_{1}\right), \quad d_{j_{1}, x_{2}}:=\sum_{j_{2}=1}^{n_{2}} c_{j_{1}, j_{2}} B_{j_{2}, p_{2}, \boldsymbol{\xi}_{2}}\left(x_{2}\right),
$$




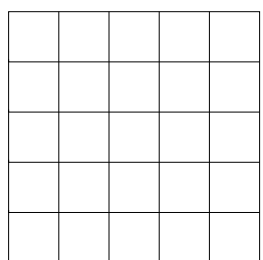

(a) initial mesh



(b) marked regions



(c) global refinement



(d) local refinement

Fig. 8 Given an initial tensor-product representation (a), an error estimator indicates regions of the mesh which require further refinement (b). The tensor-product structure necessarily implies a propagation of the refinement (c). Adaptive splines, instead, should provide a proper local control of the refinement procedure (d).

or, equivalently,

$$
s\left(x_{1}, x_{2}\right)=\sum_{j_{2}=1}^{n_{2}} d_{j_{2}, x_{1}} B_{j_{2}, p_{2}, \boldsymbol{\xi}_{2}}\left(x_{2}\right), \quad d_{j_{2}, x_{1}}:=\sum_{j_{1}=1}^{n_{1}} c_{j_{1}, j_{2}} B_{j_{1}, p_{1}, \boldsymbol{\xi}_{1}}\left(x_{1}\right) .
$$

Note that (7.9) requires $n_{1}$ univariate spline evaluations of degree $p_{2}$ and one univariate spline evaluation of degree $p_{1}$. On the other hand, (7.10) requires $n_{2}$ univariate spline evaluations of degree $p_{1}$ and one univariate spline evaluation of degree $p_{2}$. Thus, it is better to choose one of the two forms according to the minimal computational cost.

Other algorithms in the univariate B-spline setting (like knot insertion) can be extended in a similar way to the tensor-product B-spline setting.

\subsection{Local Refinement}

Despite their simple and elegant formulation, tensor-product B-spline structures have a main drawback. Any refinement of a knot sequence in one direction has a global effect in the other direction, and this prevents doing local refinement as illustrated in Figure 8.

The hierarchical spline model provides a natural strategy to guarantee the locality of the refinement. As explained in Section 6, hierarchical spline spaces are a mixture of spline spaces of different resolution, localized by the domain hierarchy. Even though the concept of hierarchical splines was detailed in the univariate setting, it can be straightforwardly extended towards the bivariate (and multivariate) setting.

When selecting a sequence of nested tensor-product spline spaces on a common basic rectangle $R$ in place of (6.1) and considering the corresponding tensor-product B-spline bases in place of (6.3), the definitions of tensor-product HB-splines and THB-splines follow verbatim Definitions 50 and 53, respectively. The properties (and their proofs) described in Section 6 also hold in the tensor-product extension. 




(a) global meshes
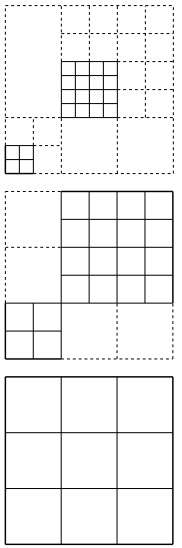

(b) local meshes

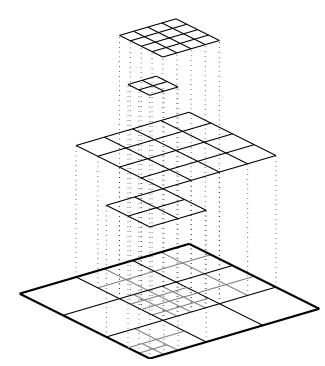

(c) domain hierarchy

Fig. 9 An example of a two-dimensional domain hierarchy consisting of three levels. The knot lines are visualized by solid lines in the domain.

We refer to $[22,23]$ for more details on tensor-product THB-splines and their properties. A full treatment of the construction of related hierarchical quasi-interpolants and their approximation properties can be found in [49,51].

Example 66. An example of a bivariate domain hierarchy together with its knot lines is illustrated in Figure 9. We consider a nested sequence of three tensor-product spline spaces defined on a (uniform) knot mesh with open knots along the boundary (Figure 9(a)). Assume the corresponding basic rectangle is denoted by $R$. Then, we can select the subsets $R=: \Omega_{1} \supseteq \Omega_{2} \supseteq \Omega_{3}$ as a union of mesh elements at each level (Figure 9(b)), and together they form the domain hierarchy $\boldsymbol{\Omega}$ (Figure 9(c)). On such domain hierarchy, we can define the corresponding HB-splines and THBsplines according to Definitions 50 and 53, respectively. Contour plots of some biquadratic tensorproduct THB-splines are depicted in Figure 10. The shape of THB-splines related to coarser levels adapts nicely to the locally refined regions in $\boldsymbol{\Omega}$, as illustrated in Figure 10(a,b). THB-splines related to the finest level are nothing else than standard tensor-product B-splines, as illustrated in Figure 10(c).

Finally, we remark that there exist also other adaptive spline models based on local tensor-product structures, like (analysis-suitable) T-splines [2, 46] and LRsplines $[9,17]$. 

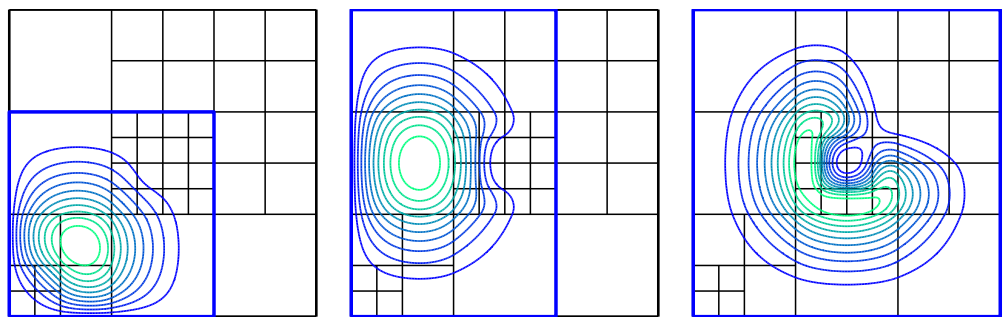

(a) level 1
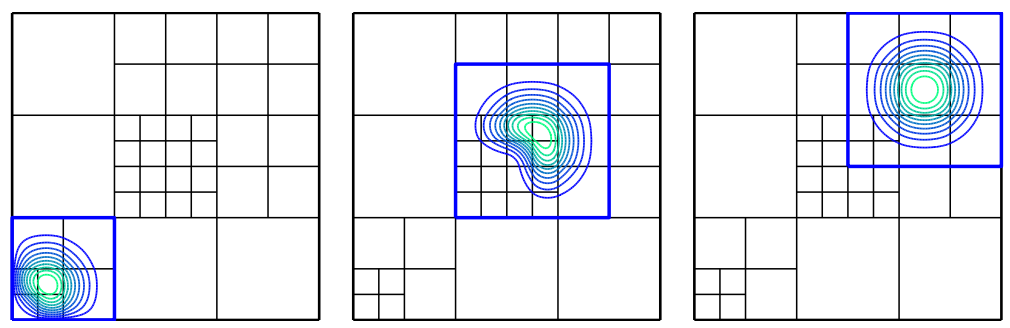

(b) level 2
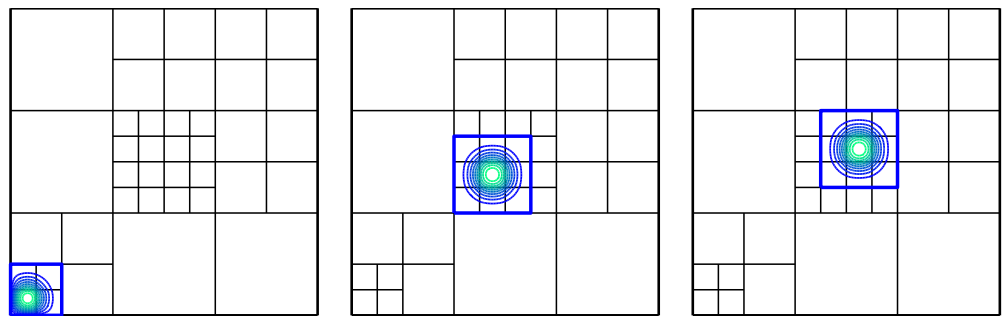

(c) level 3

Fig. 10 Contour plots of some biquadratic tensor-product THB-splines of different levels defined on the domain hierarchy given in Figure 9. The bounding box of the support of the untruncated version of each THB-spline is visualized by solid blue lines.

\section{References}

1. Beirão da Veiga, L., Buffa, A., Rivas, J., Sangalli, G.: Some estimates for $h$ - $p$ - $k$-refinement in isogeometric analysis. Numerische Mathematik 118, 271-305 (2011)

2. Beirão da Veiga, L., Buffa, A., Sangalli, G., Vázquez, R.: Analysis-suitable T-splines of arbitrary degree: Definition, linear independence and approximation properties. Mathematical Models and Methods in Applied Sciences 23, 1979-2003 (2013)

3. Böhm, W.: Inserting new knots into B-spline curves. Computer-Aided Design 12, 199-201 (1980)

4. de Boor, C.: On calculating with B-splines. Journal of Approximation Theory 6, 50-62 (1972) 
5. de Boor, C.: On local linear functionals which vanish at all B-splines but one. In: A.G. Law, N.B. Sahney (eds.) Theory of Approximation with Applications, pp. 120-145. Academic Press, New York (1976)

6. de Boor, C.: A Practical Guide to Splines, revised edn. Springer-Verlag, New York (2001)

7. de Boor, C., Fix, G.J.: Spline approximation by quasiinterpolants. Journal of Approximation Theory 8, 19-45 (1973)

8. de Boor, C., Pinkus, A.: The B-spline recurrence relations of Chakalov and of Popoviciu. Journal of Approximation Theory 124, 115-123 (2003)

9. Bressan, A.: Some properties of LR-splines. Computer Aided Geometric Design 30, 778-794 (2013)

10. Chakalov, L.: On a certain presentation of the Newton divided differences in interpolation theory and its applications. Godishnik na Sofijskiya Universitet, Fiziko-Matematicheski Fakultet 34, 353-394 (1938)

11. Cohen, E., Lyche, T., Riesenfeld, R.: Discrete B-splines and subdivision techniques in computer-aided geometric design and computer graphics. Computer Graphics and Image Processing 14, 87-111 (1980)

12. Cohen, E., Lyche, T., Schumaker, L.L.: Degree raising for splines. Journal of Approximation Theory 46, 170-181 (1986)

13. Cohen, E., Riesenfeld, R.F., Elber, G.: Geometric Modeling with Splines: An Introduction. A K Peters, Wellesley (2001)

14. Cox, M.G.: The numerical evaluation of B-splines. Journal of the Institute of Mathematics and its Applications 10, 134-149 (1972)

15. Curry, H.B., Schoenberg, I.J.: On spline distributions and their limits: The Pólya distribution functions. Bulletin of the AMS 53, 1114, Abstract 380t (1947)

16. Curry, H.B., Schoenberg, I.J.: On Pólya frequency functions IV: The fundamental spline functions and their limits. Journal d'Analyse Mathematique 17, 71-107 (1966)

17. Dokken, T., Lyche, T., Pettersen, K.F.: Polynomial splines over locally refined box-partitions. Computer Aided Geometric Design 30, 331-356 (2013)

18. Elman, H.C., Zhang, X.: Algebraic analysis of the hierarchical basis preconditioner. SIAM Journal on Matrix Analysis and Applications 16, 192-206 (1995)

19. Forsey, D.R., Bartels, R.H.: Hierarchical B-spline refinement. ACM SIGGRAPH Computer Graphics 22, 205-212 (1988)

20. Forsey, D.R., Bartels, R.H.: Surface fitting with hierarchical splines. ACM Transactions on Graphics 14, 134-161 (1995)

21. Garoni, C., Manni, C., Pelosi, F., Serra-Capizzano, S., Speleers, H.: On the spectrum of stiffness matrices arising from isogeometric analysis. Numerische Mathematik 127, 751-799 (2014)

22. Giannelli, C., Jüttler, B., Speleers, H.: THB-splines: The truncated basis for hierarchical splines. Computer Aided Geometric Design 29, 485-498 (2012)

23. Giannelli, C., Jüttler, B., Speleers, H.: Strongly stable bases for adaptively refined multilevel spline spaces. Advances in Computational Mathematics 40, 459-490 (2014)

24. Greville, T.N.E.: On the normalisation of the B-splines and the location of the nodes for the case of unequally spaced knots. In: O. Shisha (ed.) Inequalities, pp. 286-290. Academic Press, New York (1967)

25. Grinspun, E., Krysl, P., Schröder, P.: CHARMS: A simple framework for adaptive simulation. ACM Transactions on Graphics 21, 281-290 (2002)

26. Höllig, K., Hörner, J.: Approximation and Modeling with B-Splines. Society for Industrial and Applied Mathematics, Philadelphia (2013)

27. Hoschek, J., Lasser, D.: Fundamentals of Computer Aided Geometric Design. A K Peters, Wellesley (1993)

28. Kraft, R.: Adaptive and linearly independent multilevel B-splines. In: A. Le Méhauté, C. Rabut, L.L. Schumaker (eds.) Surface Fitting and Multiresolution Methods, pp. 209-218. Vanderbilt University Press, Nashville (1997)

29. Kraft, R.: Adaptive und linear unabhängige Multilevel B-Splines und ihre Anwendungen. Ph.D. thesis, University of Stuttgart (1998) 
30. Krysl, P., Grinspun, E., Schröder, P.: Natural hierarchical refinement for finite element methods. International Journal for Numerical Methods in Engineering 56, 1109-1124 (2003)

31. Lai, M.J., Schumaker, L.L.: Spline Functions on Triangulations. Cambridge University Press, Cambridge (2007)

32. Lane, J.M., Riesenfeld, R.F.: A theoretical development for the computer generation and display of piecewise polynomial surfaces. IEEE Transactions on Pattern Analysis and Machine Intelligence 2, 35-46 (1980)

33. de Laplace, P.S.: Théorie Analytique des Probabilités, third edn. Courcier, Paris (1820)

34. Lyche, T.: A note on the condition numbers of the B-spline bases. Journal of Approximation Theory 22, 202-205 (1978)

35. Manni, C., Speleers, H.: Standard and non-standard CAGD tools for isogeometric analysis: A tutorial. In: A. Buffa, G. Sangalli (eds.) IsoGeometric Analysis: A New Paradigm in the Numerical Approximation of PDEs, Lecture Notes in Mathematics, vol. 2161, pp. 1-69. Springer International Publishing (2016)

36. Marsden, M.: An identity for spline functions and its application to variation diminishing spline approximation. Journal of Approximation Theory 3, 7-49 (1970)

37. Popoviciu, T.: Sur quelques propriétés des fonctions d'une ou de deux variables réelles. Ph.D. thesis, presented to the Faculté des Sciences de Paris, published by Institutul de Arte Grafice Ardealul (Cluj, Romania) (1933)

38. Prautzsch, H., Böhm, W., Paluszny, M.: Bézier and B-Spline Techniques. Springer-Verlag, Berlin (2002)

39. Rudin, W.: Real and Complex Analysis, third edn. McGraw-Hill, Singapore (1987)

40. Scherer, K., Shadrin, A.Y.: New upper bound for the B-spline basis condition number: II. A proof of de Boor's $2^{k}$-conjecture. Journal of Approximation Theory 99, 217-229 (1999)

41. Schoenberg, I.J.: Contributions to the problem of approximation of equidistant data by analytic functions. Part A.-On the problem of smoothing or graduation. A first class of analytic approximation formulae. Quarterly of Applied Mathematics 4, 45-99 (1946)

42. Schoenberg, I.J.: Contributions to the problem of approximation of equidistant data by analytic functions. Part B.-On the problem of osculatory interpolation. A second class of analytic approximation formulae. Quarterly of Applied Mathematics 4, 112-141 (1946)

43. Schoenberg, I.J.: On spline functions. In: O. Shisha (ed.) Inequalities, pp. 255-286. Academic Press, New York (1967)

44. Schoenberg, I.J.: Cardinal interpolation and spline functions. Journal of Approximation Theory 2, 167-206 (1969)

45. Schumaker, L.L.: Spline Functions: Basic Theory, third edn. Cambridge University Press, Cambridge (2007)

46. Sederberg, T.W., Zheng, J., Bakenov, A., Nasri, A.: T-splines and T-NURCCs. ACM Transactions on Graphics 22, 477-484 (2003)

47. Sharma, A., Meir, A.: Degree of approximation of spline interpolation. Journal of Mathematics and Mechanics 15, 759-768 (1966)

48. Speleers, H.: Inner products of box splines and their derivatives. BIT Numerical Mathematics 55, 559-567 (2015)

49. Speleers, H.: Hierarchical spline spaces: Quasi-interpolants and local approximation estimates. Advances in Computational Mathematics 43, 235-255 (2017)

50. Speleers, H., Dierckx, P., Vandewalle, S.: Quasi-hierarchical Powell-Sabin B-splines. Computer Aided Geometric Design 26, 174-191 (2009)

51. Speleers, H., Manni, C.: Effortless quasi-interpolation in hierarchical spaces. Numerische Mathematik 132, 155-184 (2016)

52. Takacs, S., Takacs, T.: Approximation error estimates and inverse inequalities for B-splines of maximum smoothness. Mathematical Models and Methods in Applied Sciences 26, 14111445 (2016)

53. Vuong, A.V., Giannelli, C., Jüttler, B., Simeon, B.: A hierarchical approach to adaptive local refinement in isogeometric analysis. Computer Methods in Applied Mechanics and Engineering 200, 3554-3567 (2011)

54. Yserentant, H.: On the multilevel splitting of finite element spaces. Numerische Mathematik 49, 379-412 (1986) 